\title{
Non-Equilibrium Charge Motion in Organic Solar Cells
}

\author{
Armantas Melianas
}

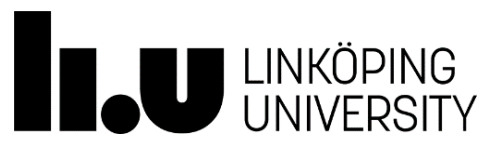

Biomolecular and Organic Electronics

Department of Physics, Chemistry and Biology (IFM) Linköping University, SE-581 83 Linköping, Sweden 


\section{Cover Image}

Simulated motion of a photo-generated electron in TQ1:PC ${ }_{71} \mathrm{BM}$, moving from top to bottom. The color changes from yellow to brown as the excess energy from the photon is gradually dissipated as heat during charge transport to the electrode, which is positioned at the bottom of the image. The cover image was designed by Armantas Melianas.

Copyright (C) Armantas Melianas

Non-Equilibrium Charge Motion in Organic Solar Cells

ISSN 0345-7524

ISBN 978-91-7685-563-8

Printed by LiU-Tryck. Linköping, Sweden, 2017 




\section{Abstract}

Organic photovoltaic (OPV) devices based on semiconducting polymers and small molecules allow for a low cost alternative to inorganic solar cells. Recent developments show power conversion efficiencies as high as $10-12 \%$, highlighting the potential of this technology. Nevertheless, further improvements are necessary to achieve commercialization.

To a large extent the performance of these devices is dictated by their ability to extract the photo-generated charge, which is related to the charge carrier mobility. Various time-resolved and steady-state techniques are available to probe the charge carrier mobility in OPVs but often lead to different mobility values for one and the same system. Despite such conflicting observations it is generally assumed that charge transport in OPV devices can be described by well-defined charge carrier mobilities, typically obtained using a single steady-state technique. This thesis shows that the relevance of such well-defined mobilities for the charge separation and extraction processes is very limited.

Although different transient techniques probe different time scales after photogeneration, they are mutually consistent as they probe the same physical mechanism governing charge motion gradual thermalization of the photo-generated carriers in the disorder broadened density of states (DOS). The photo-generated carriers gradually lose their excess energy during transport to the extracting electrodes, but not immediately. Typically not all excess energy is dissipated as the photo-generated carriers tend to be extracted from the OPV device before reaching quasi-equilibrium.

Carrier motion is governed by thermalization, leading to a time-dependent carrier mobility that is significantly higher than the steady-state mobility. This picture is confirmed by several transient techniques: Time-resolved Terahertz Spectroscopy (TRTS), Time-resolved Microwave Conductance (TRMC) combined with Transient Absorption (TA), electrical extraction of photo-induced charges (photo-CELIV). The connection between transient and steady-state mobility measurements (space-charge limited conductivity, SCLC) is described. Unification of transient opto-electric techniques to probe charge motion in OPVs is presented.

Using transient experiments the distribution of extraction times of photo-generated charges in an operating OPV device has been determined and found to be strongly dispersive, spanning several decades in time. In view of the strong dispersion in extraction times the relevance of even a well-defined time-dependent mean mobility is limited.

In OPVs a continuous 'percolating' donor network is often considered necessary for efficient hole extraction, whereas if the network is discontinuous, hole transport is thought to deteriorate significantly, limiting device performance. Here, it is shown that even highly diluted donor sites $(5.7-10 \%)$ in a buckminsterfullerene $\left(C_{60}\right)$ matrix enable reasonably efficient hole transport. Using transient measurements it is demonstrated that hole transport between isolated donor sites can occur by long-range hole tunneling (over distances of $\sim 4 \mathrm{~nm}$ ) through several $\mathrm{C}_{60}$ molecules - even a discontinuous donor network enables hole transport. 


\section{Included Papers}

[1] Unified Study of Recombination in Polymer:Fullerene Solar Cells Using Transient Absorption and Charge-Extraction Measurements

L. Mattias Andersson, Armantas Melianas, Yingyot Infahasaeng, Zheng Tang, Arkady Yartsev, Olle Inganäs, Villy Sundström

The Journal of Physical Chemistry Letters 4, 2069-2072 (2013)

[II] Dispersion-Dominated Photocurrent in Polymer:Fullerene Solar Cells

Armantas Melianas, Vytenis Pranculis, Andrius Devižis, Vidmantas Gulbinas, Olle Inganäs, Martijn Kemerink

Advanced Functional Materials 24, 4507-4514 (2014)

[III] Photo-generated Carriers Lose Energy during Extraction from Polymer-Fullerene Solar Cells

Armantas Melianas, Fabian Etzold, Tom J. Savenije, Frédéric Laquai, Olle Inganäs, Martijn Kemerink

Nature Communications 6, 8778 (2015)

[IV] Photo-generated Carrier Mobility Significantly Exceeds Injected Carrier Mobility in Organic Solar Cells

Armantas Melianas, Vytenis Pranculis, Yuxin Xia, Nikolaos Felekidis, Olle Inganäs, Vidmantas Gulbinas, Martijn Kemerink

Advanced Energy Materials DOI: 10.1002/aenm.201602143 (2017)

[V] Charge Transport in Pure and Mixed Phases in Organic Solar Cells

Armantas Melianas, Vytenis Pranculis, Donato Spoltore, Johannes Benduhn, Olle Inganäs, Vidmantas Gulbinas, Koen Vandewal, Martijn Kemerink Submitted 


\section{Short Summary of Included Papers}

[1]

[II]

[III]

[IV]

$[\mathrm{V}]$

Here, we have combined transient absorption spectroscopy and time-resolved electrical extraction measurements to monitor the charge carrier recombination dynamics in an OPV device. Under appropriate experimental conditions, the two techniques generate identical and overlapping data. We have shown that a model, incorporating the gradual thermalization of the photo-generated carriers, can explain the recombination dynamics.

Here, we have monitored the extraction of photo-generated charges from an OPV device by means of ultra-fast optical probing combined with photocurrent measurements, complemented with kinetic Monte Carlo simulations. We have shown that only charge transport models, incorporating the gradual thermalization of photo-created carriers, can describe the device photocurrent. We have outlined how the dispersion in charge extraction times can be determined in OPV devices operating under continuous illumination conditions.

Here, experimental evidence, showing the gradual thermalization of the photo-created carriers and the associated time-dependent carrier mobility, is presented. We show that photo-created carrier thermalization in OPVs is a two-step process. Most of the excess photon energy is lost by fast diffusive charge motion, followed by a slower loss during drift-dominated charge extraction. Carrier thermalization can take as long as $10 \mu$ s to complete. We have identified the time and distance scales relevant for charge extraction to show that the photo-created charges typically are extracted from the OPV device before reaching quasi-equilibrium. This paper confirms the model predictions in paper II.

Here, we have extended our analysis to measurements not involving photons. In particular, we have directly compared the mobility of photo-created charges to those that are injected from the electrodes in the dark. For the case of disordered OPVs, the mobility of photo-created charges significantly exceeds that of charges injected from the electrodes. We have explicitly calculated the error if an incorrect mobility, not accounting for carrier thermalization, is used to describe the drift of the photo-generated carriers.

Here, we have studied co-sublimed small-molecule-based donor/acceptor mixtures, where the distance between the donor sites was varied in a controlled manner. We have shown that a continuous donor network is not strictly necessary for hole transport in OPV devices. Hole hopping between isolated donor sites can occur by long-range hole tunneling through several buckminsterfullerene $\left(C_{60}\right)$ molecules, over distances as large as $\sim 4 \mathrm{~nm}$. 


\section{My Contribution to the Papers}

[1] Prepared some of the samples, performed the charge-extraction measurements, analyzed and interpreted the data together with the coauthors, helped to revise the manuscript.

[II] Coordinated the project, prepared the samples, performed the transient and the steady-state measurements, analyzed and interpreted the data together with the coauthors, wrote the manuscript draft and revised it together with the coauthors.

[III] Initiated and coordinated the project, prepared the samples, performed some of the experiments, performed the simulations, analyzed and interpreted all the data, wrote the manuscript draft and revised it together with the coauthors.

[IV] Initiated and coordinated the project, prepared the samples, performed the steady-state measurements and the simulations, supervised the transient measurements, analyzed and interpreted all the data, wrote the manuscript draft and revised it together with the coauthors.

[V] Coordinated the project, analyzed all the data, supervised the transient measurements, performed the simulations and together with the coauthors interpreted the data, wrote the manuscript draft and revised it together with the coauthors. 


\section{Papers Not-Included}

[VI] Origin of Reduced Bimolecular Recombination in Blends of Conjugated Polymers and Fullerenes

D. H. K. Murthy, Armantas Melianas, Zheng Tang, Gytis Juška, Kęstutis Arlauskas, Fengling Zhang, Laurens D. A. Siebbeles, Olle Inganäs, Tom J. Savenije

Advanced Functional Materials 23, 4262-4268 (2013)

[VII] A New Fullerene-Free Bulk-Heterojunction System for Efficient High-Voltage and High-Fill Factor Solution-Processed Organic Photovoltaics

Zheng Tang, Bo Liu, Armantas Melianas, Jonas Bergqvist, Wolfgang Tress, Qinye Bao, Deping Qian, Olle Inganäs and Fengling Zhang

Advanced Materials 27, 1900-1907 (2015)

[VIII] Comparison of Selenophene and Thienothiophene Incorporation into Pentacyclic Lactam-Based Conjugated Polymers for Organic Solar Cells

Renee Kroon*, Armantas Melianas*, Wenliu Zhuang, Jonas Bergqvist, Amaia Diaz de Zerio Mendaza, Timothy T. Steckler, Liyang Yu, Siobhan J. Bradley, Chiara Musumeci, Desta Gedefaw, Thomas Nann, Aram Amassian, Christian Müller, Olle Inganäs, Mats R. Andersson.

Polymer Chemistry 6, 7402-7409 (2015)

*Shared first author

[IX] High-Entropy Mixtures of Pristine Fullerenes for Solution-Processed Transistors and Solar Cells

Amaia Diaz de Zerio Mendaza, Armantas Melianas, Stephan Rossbauer, Olof Bäcke, Lars Nordstierna, Paul Erhart, Eva Olsson, Thomas D. Anthopoulos, Olle Inganäs, Christian Müller

Advanced Materials 27, 7325-7331 (2015)

Journal cover 
[X] Fully-Solution-Processed Organic Solar Cells with a Highly Efficient Paper-Based Light Trapping Element

Zheng Tang, Anders Elfwing, Armantas Melianas, Jonas Bergqvist, Qinye Bao, Olle Inganäs

Journal of Materials Chemistry A 3, 24289-24296 (2015)

[XI] New Method for Lateral Mapping of Bimolecular Recombination in Thin-Film Organic Solar Cells

Jonas Bergqvist, Wolfgang Tress, Daniel Forchheimer, Armantas Melianas, Zheng Tang, David Haviland, Olle Inganäs

Progress in Photovoltaics: Research and Applications 24, 1096-1108 (2016)

[XII] Role of Coherence and Delocalization in Photo-Induced Electron Transfer at Organic Interfaces

Vytautas Abramavičius, Vytenis Pranculis, Armantas Melianas, Olle Inganäs, Vidmantas Gulbinas, Darius Abramavičius

Scientific Reports 6, 32914 (2016)

[XIII] Nonequilibrium Drift-Diffusion Model for Organic Semiconductor Devices Nikolaos Felekidis, Armantas Melianas, Martijn Kemerink

Physical Review B 94, 035205 (2016)

[XIV] A Fullerene Alloy Based Photovoltaic Blend with a Glass Transition above $200{ }^{\circ} \mathrm{C}$ Amaia Diaz de Zerio Mendaza, Armantas Melianas, Ferry A. A. Nugroho, Olof Bäcke, Eva Olsson, Christoph Langhammer, Olle Inganäs, Christian Müller Journal of Materials Chemistry A 5, 4156-4162 (2017) 



\section{Contents}

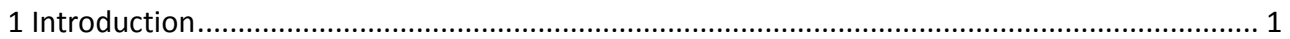

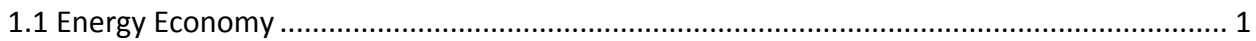

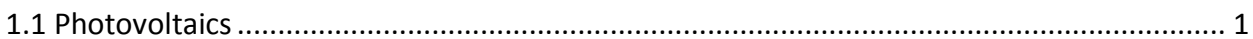

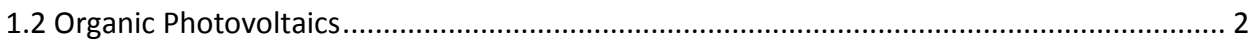

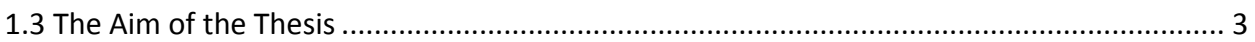

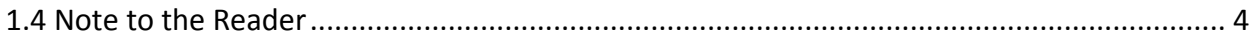

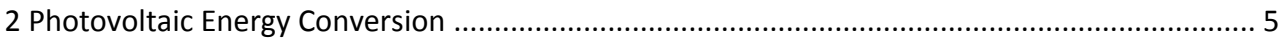

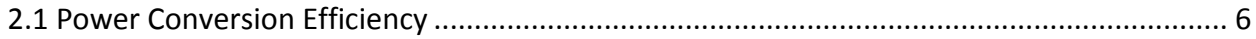

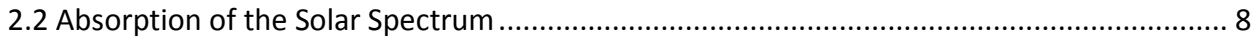

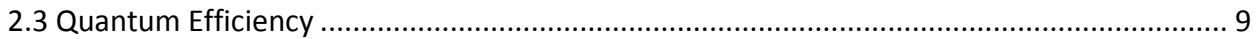

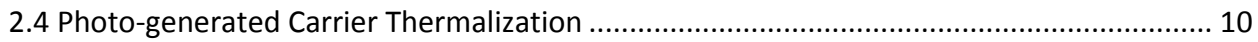

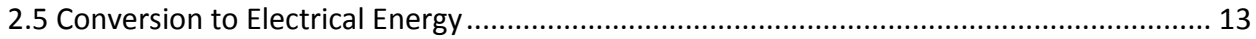

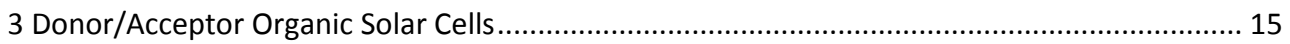

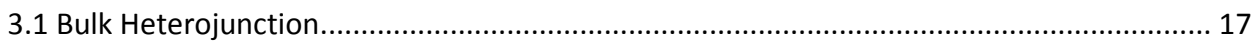

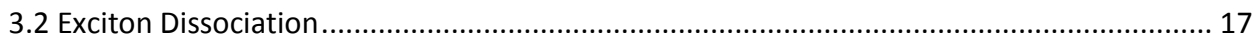

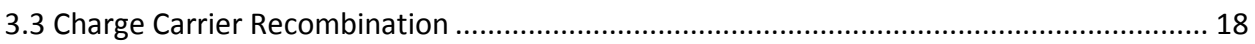

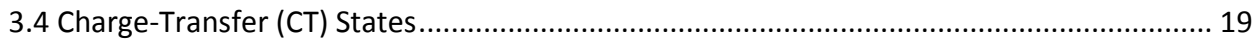

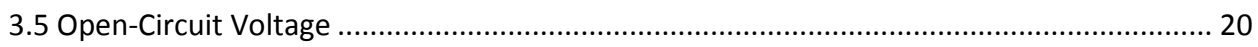

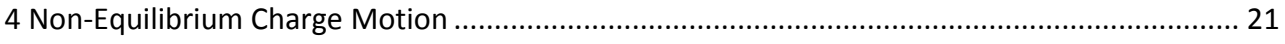

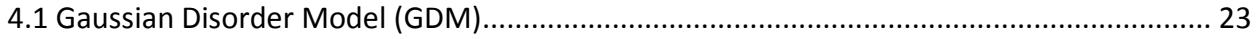

4.2 Thermalization According to the GDM framework ........................................................... 26

5 General Considerations for Time-resolved Measurements ..................................................... 29

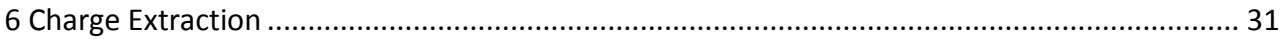

6.1 TREFISH Combined with Transient Photocurrent Measurements ..................................... 31

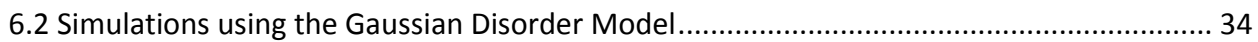

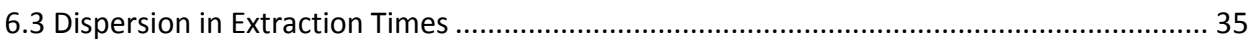

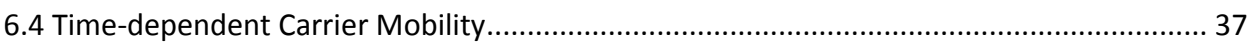

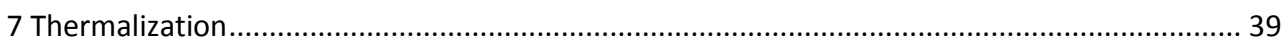

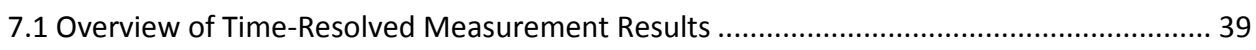




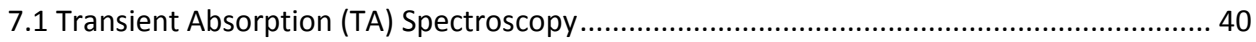

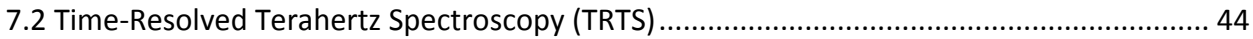

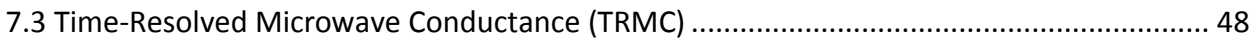

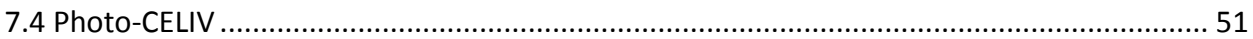

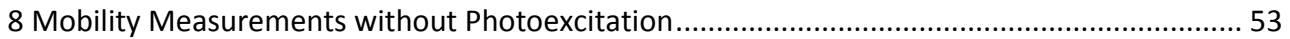

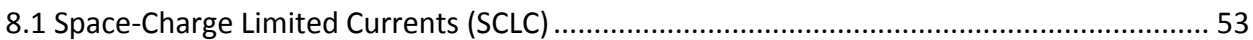

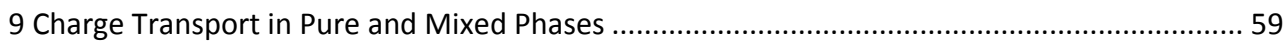

9.1 Relation between Phase Purity and Charge Transport .......................................................... 59

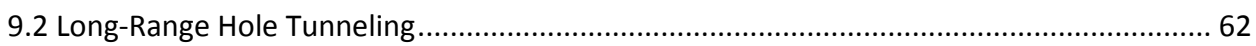

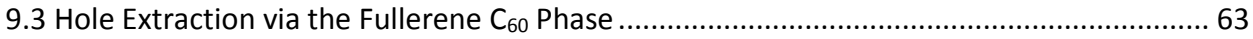

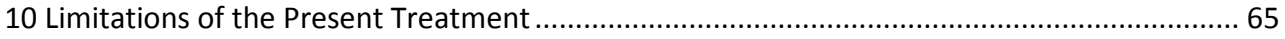

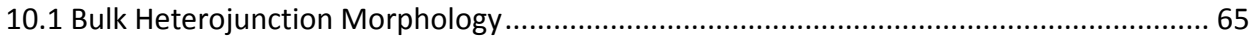

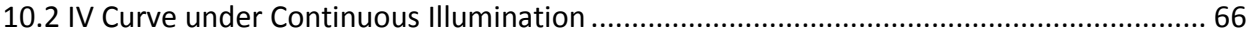

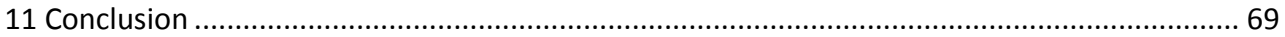

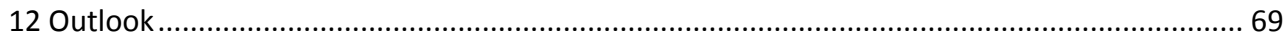

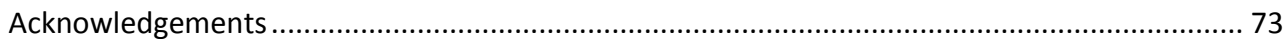

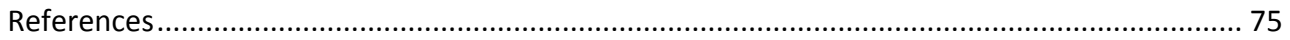

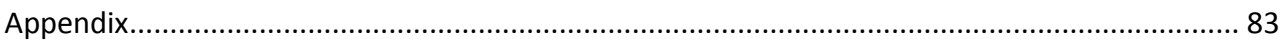





\section{Introduction}

\subsection{Energy Economy}

The energy economy of the world is currently mainly based on the use of fossil fuels, such as coal, oil and natural gas. Fossil fuels form by natural processes over a period of millions of years and have a finite reserve. There are two major problems in using a finite energy source to satisfy our needs. First of all, fossil fuels will eventually run out - they are non-renewable, meaning that our energy economy is not sustainable. Secondly, the combustion of fossil fuels consumes oxygen $\left(\mathrm{O}_{2}\right)$ and, among other harmful compounds, releases the greenhouse gas, carbon dioxide $\left(\mathrm{CO}_{2}\right)$. Triatomic molecules, such as $\mathrm{CO}_{2}$, are good absorbers in the infrared and absorb a fraction of the heat emitted by the surface of the earth. Increasing $\mathrm{CO}_{2}$ content enhances absorption and heating of the atmosphere, which in turn leads to increased heat emission back to the surface of the earth, causing global warming. Experts seem to agree that a rise in global-mean temperature should not exceed more than $2{ }^{\circ} \mathrm{C}$ above pre-industrial level. While this may not occur during our lifetime, we must consider how life will continue for the future generations. Well before our fossil fuel reserves are depleted, we must begin to develop sustainable and environmentally friendly alternatives.

\subsection{Photovoltaics}

Solar energy is renewable, environmentally friendly and abundant. The power from the sun, incident on the surface of the earth after passing the earth's atmosphere, is practically unlimited ( 120 000 TW) compared to current human needs ( 20 TW). The irradiance from the sun at the surface of the earth is roughly $1000 \mathrm{~W} \mathrm{~m}^{-2}$. Nevertheless, even today, we are making little use of solar energy in terms of electricity production (Figure 1). Electricity, after all, is one of the most useful forms of energy, as it can be used for almost everything.

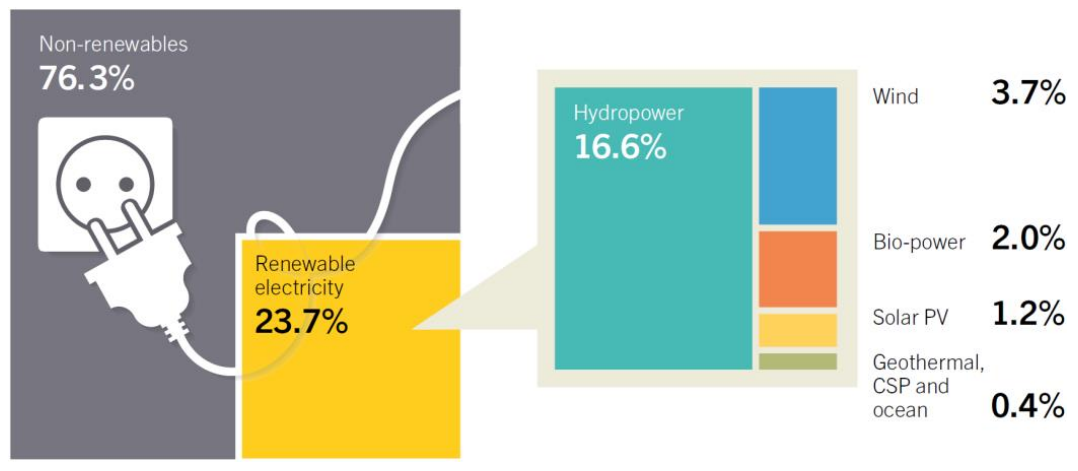

Figure 1. Estimated renewable energy share of global electricity production at year-end 2015. Adapted from the Renewables 2016 Global Status Report ${ }^{1}$. 
The conversion of solar energy to electrical energy can be achieved by the use photovoltaic modules. Current photovoltaic market is dominated by wafer-based crystalline silicon modules, with a market share of $>90 \%$ (refs 2,3 ). Since the advent of the silicon solar cell from the Bell labs in the 1950s, the field has seen tremendous progress, leading to commercial silicon-based module power conversion efficiencies in the range of $16-21 \%$ (ref. 2) (for a single-junction crystalline silicon solar cell the theoretical maximum is about $30 \%)^{4}$. Continually falling prices and improvements in module efficiency are expected to soon make photovoltaics as one of the lowest cost options for future electricity production.

While silicon has evident advantages for use in photovoltaics (it is the second most abundant element on earth, it is robust and the material itself is non-toxic), it requires relatively complex and energy-intensive manufacturing steps. The development of organic, i.e. carbon-based, semiconducting polymers and small molecules offers a promising alternative.

\subsection{Organic Photovoltaics}

Organic semiconductors are generally non-toxic and offer potentially easy means of solar cell fabrication by solution processing at low temperatures. As such, large areas of photovoltaic modules can, at least in principle, be rapidly deposited from inks by roll-to-roll techniques, at low monetary and energy cost. These are soft materials with strong absorption coefficients, enabling lightweight and flexible organic solar cells (less than $1 \mu \mathrm{m}$ thick films are sufficient to absorb most of the incident light). Desirable material properties can be almost endlessly fine-tuned by chemical synthesis and new, better compounds are discovered on a continuous basis. As such, solar cells based on organic semiconductors have many of the necessary features for a successful low-cost photovoltaic technology.

Current organic solar cell power conversion efficiencies for small lab-scale devices are in the range of $10-12 \%$ (refs $5-7$ ) and the technology is currently being upscaled to larger-area photovoltaic modules. However, present organic solar cell materials suffer from two major limitations. First of all, organic solar cells degrade rather rapidly. Secondly, organic solar cell efficiencies remain too low for commercial applications. A similar situation has occurred in the past for hydrogenated amorphous silicon (a-Si:H) solar cells, which is also an environmentally benign technology, allowing for low-temperature and low-cost deposition on flexible substrates. However, the relatively low efficiencies $(\sim 10 \%)$ and degradation have limited the market for a-Si:H mainly to pocket calculators. As high power conversion efficiencies at low-cost continue to be the main driving force for photovoltaic technologies to enter the market, organic solar cell materials must be improved.

Although present organic photovoltaic (OPV) devices are reasonably efficient in terms of light in-coupling and photocurrent generation, they are quite poor in terms of making use of the absorbed photon energy. In other words, a large portion of the photon's energy is wasted during 
the conversion from solar to electrical energy, thus limiting photovoltaic performance. These losses occur gradually, following the absorption of light, with different processes taking place at different time scales. In this thesis, these processes were probed by transient measurements.

\subsection{The Aim of the Thesis}

Much of the physics describing the operation of OPV devices has been adapted from inorganic solar cells. In conventional inorganic materials, the photo-generated carriers are assumed to be in thermal equilibrium with the lattice ${ }^{8,9}$. This assumption is experimentally justified as photo-generated carrier thermalization completes very rapidly ${ }^{10,11}$, i.e. before any significant carrier transport has occurred. In other words, a quasi-equilibrium situation is quickly established and the photo-generated carrier populations can be conveniently described using Fermi-Dirac statistics. Following thermalization, the photo-generated carriers are transported at their quasi-equilibrium energies with a well-defined carrier mobility. Despite lack of conclusive proof and conflicting evidence in literature this is often assumed to be also the case in OPV devices.

Previous experimental and theoretical studies show strong indications that photo-generated carrier thermalization in organic semiconductors is, in fact, relatively slow ${ }^{12-15}$. Furthermore, reported charge carrier mobility values typically differ by orders of magnitude when probed at different time scales following photoexcitation ${ }^{13-20}$. Although most researchers in the field are aware of this and would agree that the motion of photo-generated charges in OPV devices should be described as a non-equilibrium transient process, the assumptions of quasi-equilibrium and well-defined carrier mobilities remain widely used in the OPV community. This is largely due to a lack of a comprehensive experimental study. Transient measurements are typically discussed in isolation, whereas all of the relevant time scales for charge transport must be investigated.

The aim of this is thesis is provide a comprehensive quantitative study involving multiple experimental techniques and simulations, spanning the relevant time scales for charge transport in OPVs. The main questions that this thesis aims to answer are:

- What are the relevant time and distance scales for photo-generated carrier thermalization in OPV devices? What is the main mechanism via which photo-generated carrier thermalization takes place? Can thermalization be avoided?

- If thermalization is sufficiently slow, can the photo-generated carriers be extracted from the OPV device before reaching quasi-equilibrium?

- Can charge transport in OPV devices be described by well-defined charge carrier mobilities? If not, what is the alternative? 
This thesis shows that photo-generated carrier transport in OPV devices is completely dominated by non-equilibrium effects, governed by the gradual thermalization of the photo-generated carriers. There is no unique mobility. The mobility of photo-created charges is strongly time-dependent, as it gradually decreases following photoexcitation. The photo-generated carriers are typically extracted from the OPV device before reaching quasi-equilibrium.

\subsection{Note to the Reader}

Chapters 2 and 3 provide a brief introduction to photovoltaic energy conversion and organic solar cells, respectively. Readers familiar with these topics can skip these chapters and start reading at chapter 4, which describes the theoretical framework used to explain the transient measurements discussed in the rest of the thesis. Chapter 5 highlights some important aspects regarding time-resolved measurements and the choice of samples. Chapters 6-9 summarize the main findings of the papers. The papers occupy the second half of the thesis and can be found after the Appendix.

Chapter 6 describes the charge extraction experiments (paper II). Chapter 7 describes the experimental data on photo-generated carrier thermalization (papers I and III). Chapter 8 compares mobility measurements with and without photoexcitation (paper IV). Chapter 9 extends the results in the previous chapters to measurements on small-molecule-based OPV blends with a well-defined morphology (paper V). Chapter 10 discusses some modeling limitations and shows ongoing work to circumvent those limitations. Chapters $11-12$ conclude the thesis and provide some ideas for future studies.

Chemical structures and full names of the materials studied in this thesis are given in the Appendix. Unless otherwise noted, the discussion centers around the main system studied in this thesis (TQ1:PC $71 B M)$. 


\section{Photovoltaic Energy Conversion}

Photovoltaic energy conversion relies on the absorption of photons by a semiconductor (Figure 2). Only photons with an energy higher than the semiconductor bandgap are absorbed, the rest are either reflected or transmitted. The absorption of a photon with an energy higher than the semiconductor bandgap promotes an electron from the valence band to the conduction band, generating an electron-hole pair. If such an electron-hole pair is sufficiently long-lived, i.e. does not rapidly decay to the ground state, the photon energy, stored in the electron-hole pair, can be converted into electrical energy by the use of a photovoltaic device.

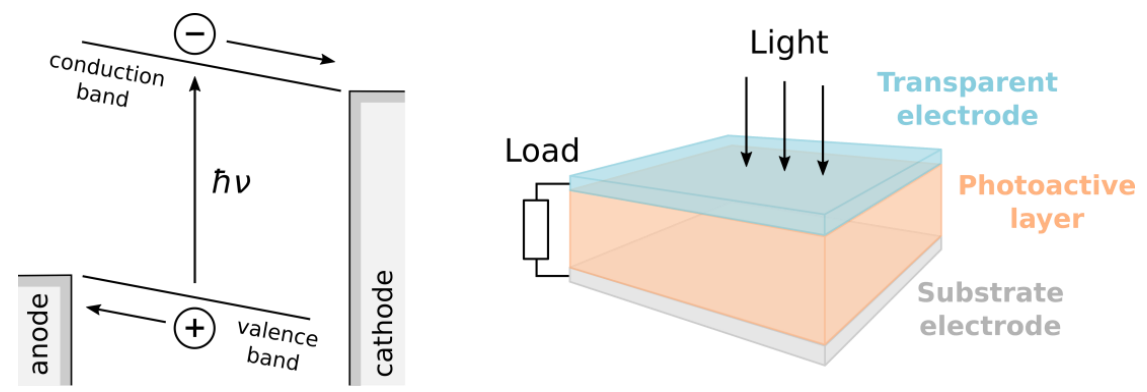

Figure 2. Schematic of the basic operating mechanism of a single-junction photovoltaic device.

The simplest photovoltaic device consists of a semiconducting photoactive layer sandwiched between two electrodes. This type of design is commonly referred to as the single-junction solar cell. In organic solar cells the photoactive layer is based on semiconducting polymers and/or small molecules. Light enters through the transparent electrode and is absorbed by the photoactive layer. The substrate electrode is typically reflective, e.g. a metal. This way the photons that are not absorbed by the photoactive layer during the first pass are reflected and traverse the photoactive layer again - more photons are absorbed in the semiconductor. The photo-generated charges are then collected at the electrodes.

To produce work in an external circuit (represented by the load in Figure 2), the extraction of holes and electrons must be selective at each electrode. In other words, the photovoltaic device must be engineered with an asymmetry so that the photo-generated electrons are preferentially collected at the cathode, whereas the photo-generated holes are collected at the anode. In inorganic solar cells the asymmetry is typically introduced by inhomogeneous doping of the semiconductor, the classic example is the $p-n$ junction. In organic solar cells the asymmetry is introduced by the use of electrodes with different work functions, which creates a built-in bias $\left(U_{\mathrm{bi}}\right)$, i.e. a built-in electric field in the photoactive layer, driving the extraction of photo-generated holes and electrons at their respective electrodes. Due to the asymmetry, the photovoltaic device acts as a diode (Figure 3 ). 


\subsection{Power Conversion Efficiency}

The amount of electrical power that the photovoltaic device generates upon illumination is a product of photocurrent and photovoltage that act on the external load. For a fixed light intensity, the photovoltaic device produces maximum power $\left(P_{\max }\right)$ when this load is optimized. The optimum load can be determined by recording the Current-Voltage (IV) characteristic of the photovoltaic device under illumination (Figure 3 ). In this case the applied voltage sweep effectively simulates the presence of different loads. The Maximum-Power Point (MPP) marks the conditions for an optimum load.

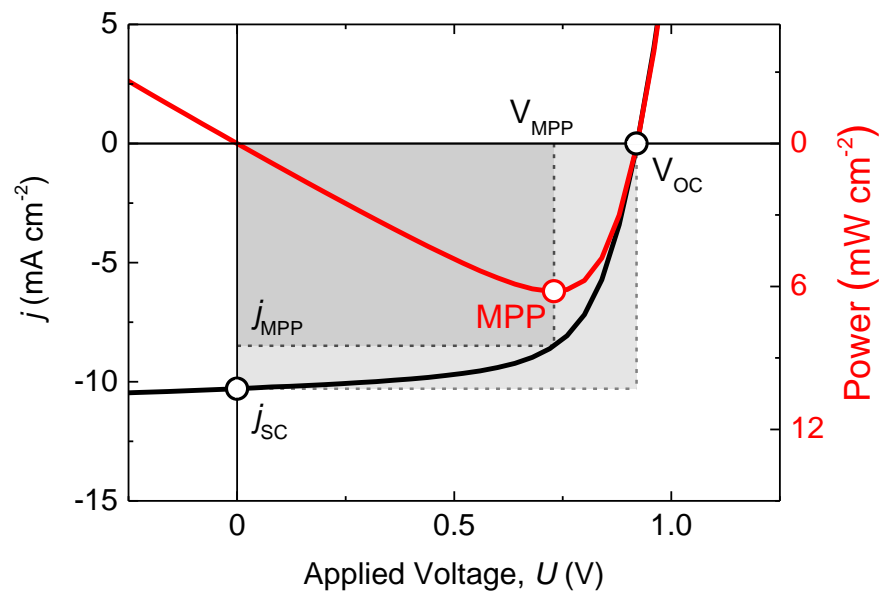

Figure 3. Current-Voltage (IV) characteristic of a TQ1:PC $\mathrm{P}_{71} \mathrm{BM}$ organic photovoltaic device.

The power conversion efficiency (PCE) of the photovoltaic device is typically estimated at the maximum-power point. The PCE is defined as the ratio between the maximum output power $\left(P_{\max }\right)$ of the photovoltaic device and the power of the incident light $\left(P_{\text {in }}\right)$

$$
P C E=\frac{P_{\text {max }}}{P_{\text {in }}}=\frac{j_{M P P} V_{M P P}}{P_{\text {in }}}
$$

where $j_{\text {MPP }}$ and $V_{\text {MPP }}$ refer to the output photocurrent density and the applied voltage at the maximum-power point, respectively.

Nevertheless, it is often useful to know how the measured solar cell compares to an idealized solar cell. Hence, the PCE is often defined by other points on the IV curve. In particular, with respect to those where the output power is equal to zero. This occurs at two points on the IV curve. The point where the applied voltage is equal to zero corresponds to short-circuit conditions, e.g. no load in the external circuit. The photocurrent density recorded at this point is referred to as the short-circuit density (jsc). When the applied voltage is such that the recorded photocurrent is equal to zero, e.g. an infinitely high load, this point is referred to as the open-circuit voltage $\left(V_{O C}\right)$. The 
product of short-circuit density and open-circuit voltage define the maximum power attainable by an idealized solar cell.

The ratio

$$
F F=\frac{j_{M P P} V_{M P P}}{j_{S C} V_{O C}}
$$

is referred to as the fill factor $(F F)$ and quantifies how close to ideal the measured solar cell is (given its $j_{s c}$ and $V_{o c}$ ). A perfect solar cell would have a square-like IV curve. In that sense, the $F F$ quantifies the 'squareness' of the IV curve.

Using these quantities the PCE is then

$$
P C E=\frac{F F j_{S C} V_{O C}}{P_{\text {in }}}
$$

The added benefit of this definition is that all three IV parameters are related to the physical properties of the photoactive layer. As will be explained in the next section, the short-circuit density (jsc) depends on the number of photo-generated charges that are successfully extracted from the photovoltaic device. The open-circuit voltage $\left(V_{o c}\right)$ is related to the fraction of the photon's energy that the photovoltaic device converts to electrical energy. Any photovoltaic device that makes the best use of the incident photons has a high power conversion efficiency. 


\subsection{Absorption of the Solar Spectrum}

To compare the performance of different photovoltaic technologies, a standard solar illumination spectrum is used for device characterization, commonly referred to as the AM1.5g solar spectrum (Figure 4). The label AM1.5g refers to absorption losses, e.g. due to water and oxygen, as sunlight passes the earth's atmosphere; and a correction for the yearly-averaged angle of incidence at mid-latitudes.

To make the most use of the incident solar spectrum, the thickness and the absorption of each layer in the photovoltaic device stack must be tailored for maximum absorption in the photoactive layer. The absorption coefficient of organic semiconductors is generally very high $\left(\sim 10^{4} \mathrm{~cm}^{-1}\right)$, hence, a photoactive film that is just a few hundred nanometers thick is sufficient to absorb most of the photons above the optical gap of the photoactive layer. Thick photoactive layers are more desirable as they absorb more light, however, the optimal thickness is also set by electron-hole recombination, as described in the next section. The optimal photoactive layer thickness in OPV devices is typically $\sim 100 \mathrm{~nm}$.

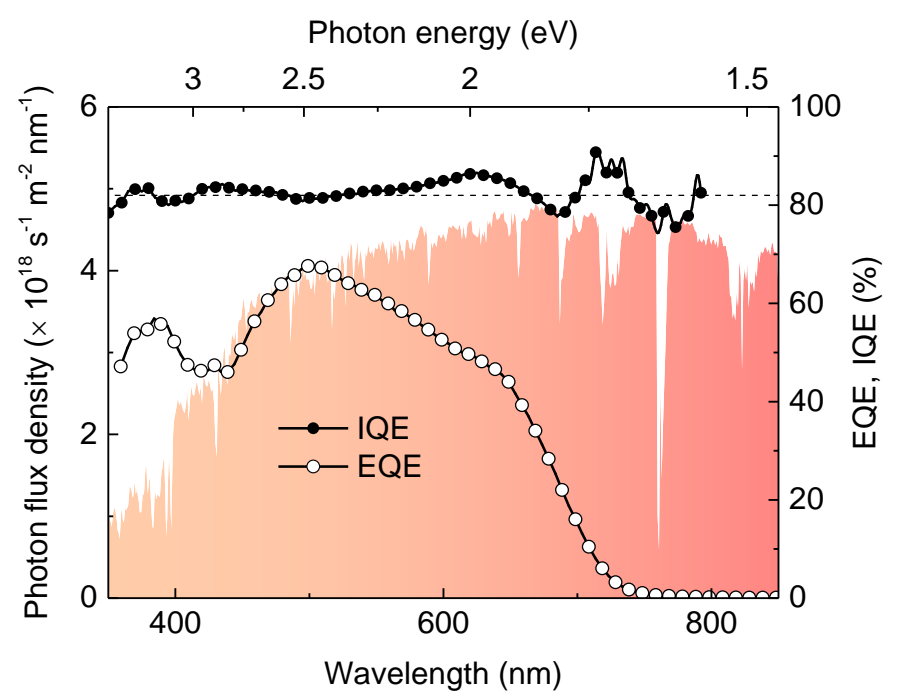

Figure 4. The AM1.5g solar spectrum (shaded area) and the internal and external quantum efficiencies (IQE and EQE, respectively) of an optimized TQ1:PC ${ }_{71} B M$ organic photovoltaic device. 


\subsection{Quantum Efficiency}

The ratio of the number of photons incident on the photovoltaic device $\left(n_{p h}^{i n}\right)$ to the number of extracted electrons $\left(n_{e}^{\text {out }}\right)$ defines the External Quantum Efficiency (EQE) of the photovoltaic device

$$
E Q E(\lambda)=\frac{n_{e}^{\text {out }}(\lambda)}{n_{p h}^{\text {in }}(\lambda)}
$$

which is typically measured at short-circuit conditions $(U=0 \mathrm{~V})$. This is important as, contrary to conventional inorganic solar cells, the EQE of an organic solar cell can be bias-dependent ${ }^{21}$.

EQE is lower than $100 \%$ due to optical and electron-hole recombination losses. For example, a fraction of the incident photons may be lost to parasitic absorption in the electrodes. An additional fraction of the incident photons may be simply reflected by or transmitted through the photovoltaic device. In either case, optical losses decrease the number of extracted charges per incident photon, reducing the EQE of the photovoltaic device. Furthermore, not all of the photons absorbed in the photoactive layer may be successfully converted to extracted charges - a fraction of initial photoexcitations may decay to the ground state by electron-hole recombination, which further reduces the EQE of the photovoltaic device.

Both optical and recombination losses affect the short-circuit density and thus the power conversion efficiency of the photovoltaic device. The EQE relates to short-circuit density as

$$
j_{S C}=-e \int n_{p h}^{A M 1.5}(\lambda) E Q E(\lambda) d \lambda
$$

where $e$ is the elementary charge and $n_{p h}^{A M 1.5}(\lambda)$ is the photon flux density of the AM1.5g solar spectrum. Figure 4 shows the EQE of an optimized organic photovoltaic device based on the main material system investigated in this thesis (TQ1:PC ${ }_{71} B M$ ). The EQE drops to $0 \%$ at $750-800 \mathrm{~nm}$ due to the optical gap of TQ1:PC ${ }_{71} \mathrm{BM}$. The short-circuit density is $j_{\mathrm{SC}}=-10 \mathrm{~mA} \mathrm{~cm}{ }^{-2}$.

The ability of the photovoltaic device to successfully convert the photons absorbed in the photoactive layer to extracted charge carriers is characterized by the Internal Quantum Efficiency (IQE) of the device. In this case, only the photons that are absorbed by the photoactive layer $\left(n_{p h}^{a b s}\right)$ are counted

$$
\operatorname{IQE}(\lambda)=\frac{n_{e}^{o u t}(\lambda)}{n_{p h}^{a b s}(\lambda)}
$$

This way losses to charge carrier recombination can be quantified. The IQE of optimized organic solar cells is generally higher than $80 \%$, regardless of the absorbed photon energy ${ }^{22}$. This is also the case for the optimized TQ1:PC ${ }_{71} \mathrm{BM}$ device, with a mean IQE $\approx 83 \%$. Hence, in this case, $\approx 17 \%$ of the photons absorbed in the TQ1:PC $71 \mathrm{BM}$ photoactive layer are lost to charge carrier recombination, whereas the remaining majority of the absorbed photons $(\approx 83 \%)$ are successfully extracted as charges. 


\subsection{Photo-generated Carrier Thermalization}

In an inorganic solar cell, following the absorption of a high energy photon, the photo-generated electron initially resides at an energy higher than the conduction band edge (Figure 5). The same is true for the photo-generated hole, which is initially situated at an energy higher than the valence band edge. Both the electron and the hole are thus initially situated at relatively high-energy sites in their respective Density of States (DOS).
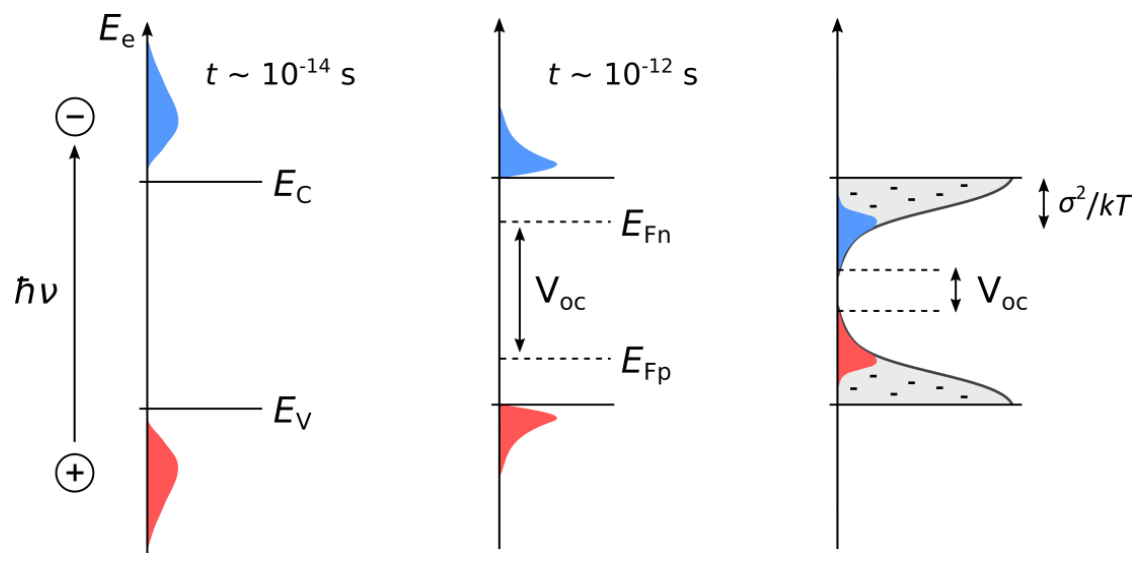

Figure 5. Photo-created electrons and holes rapidly lose energy by thermalization. The presence of localized sites may lead to further thermalization (depends on carrier trap/release rates).

Since there are many DOS sites at a lower energy, the excess energy from the photon is quickly dissipated as heat by a step-wise release of phonons. The photo-generated carriers thermalize to their respective band edge. Thermalization typically completes in less than a picosecond $d^{10,11}$. Therefore, any photovoltaic device that would make use of the energy stored in the non-thermalized carrier populations, i.e. a hot carrier solar cell, must extract the photo-generated carriers before they thermalize, e.g. within a time scale shorter than $\sim 1 \mathrm{ps.} \mathrm{A} \mathrm{hot} \mathrm{carrier} \mathrm{solar} \mathrm{cell}$ with a power conversion efficiency relevant for practical applications has not been demonstrated yet $^{23}$. Hence, in conventional solar cells, the excess photon energy is simply lost to thermalization. This is one of the main losses in single-junction photovoltaic devices. The remaining electron-hole pair energy that the photovoltaic device can convert to electrical energy is then set by the bandgap of the semiconductor.

A high bandgap semiconductor would have minimal thermalization losses at the expense of absorbing very few photons (the incident solar spectrum contains few photons at high photon energies). In contrast, a low bandgap semiconductor would absorb a large portion of the solar spectrum, but the remaining energy per electron-hole pair would be reduced significantly by large losses to thermalization. There exists an optimum bandgap, first derived by Shockley and Quisser $\left(E_{\mathrm{g}} \approx 1.0-1.5 \mathrm{eV}\right)^{4}$. Therefore, any material intended for photovoltaic applications should have a 
similar optical gap. The highest efficiency inorganic solar cell materials, e.g. crystalline silicon $\left(E_{\mathrm{g}}=1.1 \mathrm{eV}\right)$ and Galium Arsenide $(\mathrm{GaAs})\left(E_{\mathrm{g}}=1.4 \mathrm{eV}\right)$, have the desirable bandgap. Materials intended for OPV are tailored by chemical synthesis to have an optical gap that maximizes the power conversion efficiency.

Even at the close-to optimum bandgap, loses to thermalization are significant. Figure 6 illustrates the fraction of the solar spectrum irradiance that is converted by an idealized solar cell with a bandgap of crystalline silicon $\left(E_{\mathrm{g}}=1.1 \mathrm{eV}\right)$. The solar cell is assumed to convert every single photon that is absorbed to electrical power, only losses to thermalization and to the transmission of photons are accounted for. In real crystalline silicon (c-Si) solar cells, thermalization and transmission losses reduce the maximum attainable electrical power by approximately $33 \%$ (thermalization) $+26 \%$ (transmission $)=59 \%(\text { total })^{9}$. These are the main losses in optimized single-junction photovoltaic devices.

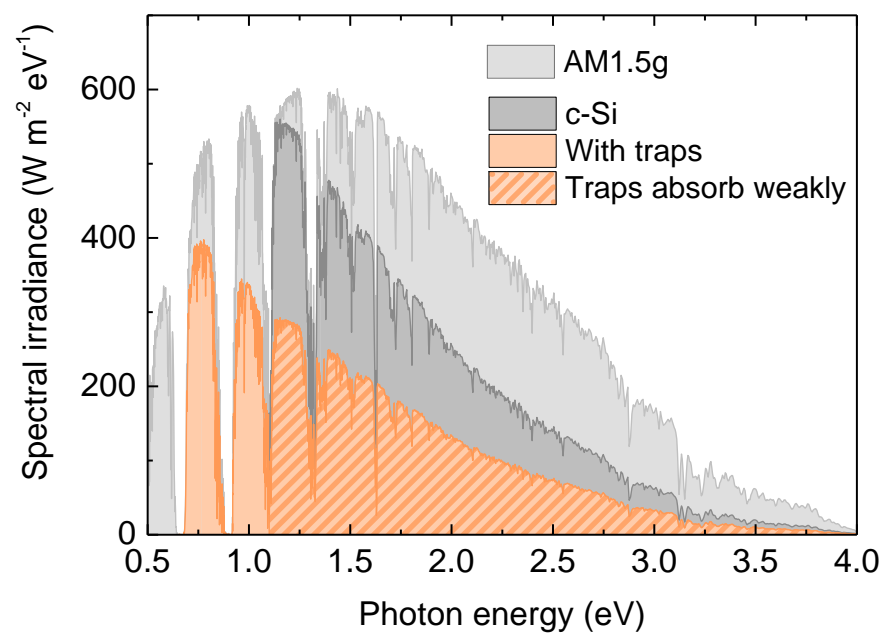

Figure 6. The fraction of the incident AM1.5g solar spectrum (light grey), converted to electrical power by an idealized solar cell with a bandgap of c-Si (dark grey), is reduced when localized sites are present in the bandgap (orange). Generally, localized sites are weakly absorbing, reducing the maximum attainable power further (orange patterned).

Amorphous semiconductors, e.g. amorphous silicon (a-Si), also contain localized trap states that extend into the bandgap ${ }^{24,25}$. The photo-generated carriers may then thermalize to these low lying $\operatorname{sites}^{26}$ (Figure 5). The exact energetic position of the photo-generated carriers in an illuminated semiconductor with an arbitrary distribution of localized sites has been derived analytically by Simmons et al. (ref. 27). Regardless of the trap distribution, this effectively lowers the bandgap of the semiconductor - thermalization to low lying sites reduces the maximum attainable electrical energy per electron-hole pair ${ }^{28}$ (Figure 6). 
For a Gaussian distribution of traps with a standard deviation of $\sigma$, the mean energy of the thermalized carrier distribution in the Boltzmann limit, i.e. in the limit of low carrier densities, is positioned at $\sigma^{2} / k T$ below the band edge (Figure 5 ). For disordered organic solar cells this typically amounts to $\sigma^{2} / k T \approx 100-400 \mathrm{meV}$, and is one of the main factors limiting the power conversion efficiency of organic solar cells ${ }^{29}$.

Generally speaking, absorption via localized sites is very weak, e.g. this is well known for a-Si ${ }^{24,25}$. In this case the semiconductor effectively has the same optical gap as without localized sites, however, the photo-generated carriers can thermalize below the optical gap. The net effect is an overall similar absorption of the solar spectrum but a reduced electron-hole pair energy. This reduces the maximum attainable electrical power even further (Figure 6 patterned area).

Therefore, any material disorder leading to localized sites below the band edges is undesirable for photovoltaic applications ${ }^{27-29}$. Unfortunately, organic solar cells are quite disordered and also display weak optical transitions below the optical gap. In organic solar cells weak optical transitions occur by the absorption of photons in the charge-transfer (CT) state manifold, as described in the chapter on organic solar cells (chapter 3 ). 


\subsection{Conversion to Electrical Energy}

The photovoltaic device converts the electron-hole pair energy, set by the energy of the hole and the electron in their respective DOS, to electrical energy according to the laws of thermodynamics ${ }^{9}$. The conversion of electron-hole pair energy to electrical energy leads to entropy production ${ }^{9,30}$. Hence, the actual electrical energy that the photovoltaic device can deliver, when a photo-generated electron-hole pair is extracted, is less than the difference in the electron and hole energies (to be precise, less than the total enthalpy of the electron-hole pair). A fraction of the photon's energy is thus lost to entropy production.

By considering a semiconductor under steady-state illumination, it can be shown that the maximum amount of electrical energy that the photovoltaic device can harvest from the photo-generated electron-hole pairs is related to the quasi-Fermi level splitting under open-circuit conditions $^{9}$

$$
E_{F n}-E_{F p}=E_{e f f}-k T \ln \left(\frac{N_{C B} N_{V B}}{n_{e} n_{h}}\right)=e V_{O C}
$$

where $e$ is the elementary charge, $E_{\mathrm{Fn}}$ and $E_{\mathrm{Fp}}$ are the quasi-Fermi levels for the photo-generated electron and hole populations, respectively, $E_{\text {eff }}$ is the effective bandgap of the semiconductor, $n_{\mathrm{e}}$ and $n_{\mathrm{h}}$ are the photo-generated electron and hole densities, respectively, $N_{\mathrm{CB}}$ and $N_{\mathrm{VB}}$ are the effective density of states in the conduction and valence bands, respectively. The quasi-Fermi level splitting at open-circuit conditions and thus the maximum electrical energy that the photovoltaic can harvest under continuous illumination is indicated in Figure 5.

The splitting of the quasi-Fermi levels is equal to the open-circuit voltage $\left(V_{o c}\right)$ of a continuously illuminated photovoltaic device. Hence, the $V_{o c}$ of an illuminated solar cell can be determined by either an IV scan or by a simple measurement using a voltmeter, which acts as an infinite load.

The amount of energy lost to entropy production is set by the effective density of states in the valence and conduction bands ( $N_{\mathrm{VB}}$ and $N_{\mathrm{CB}}$, respectively), which are complicated functions of energy ${ }^{30}$. The effective density of states are clearly defined only for semiconductors where the photo-generated carriers can be described using effective masses, which is not the case for disordered organic solar cells. Nevertheless, organic solar cells generally follow the same $V_{o c}$ dependence on temperature and carrier density. The analytical expression describing the open-circuit voltage in organic solar cells has been first derived and experimentally tested by K. Vandewal et al. (refs 31,32).

Note that $V_{o c}$ is logarithmically dependent on carrier density. Thus, materials with low charge carrier recombination losses are desirable for photovoltaic applications ${ }^{33}$. 



\section{Donor/Acceptor Organic Solar Cells}

The photoactive layer in the earliest generation of OPV devices was based on a single organic semiconductor material, sandwiched between a transparent and a reflective electrode, in analogy to inorganic solar cells (Figure 7). However, the power conversion efficiency of such devices is generally very low, mainly due to the large exciton binding energy in organic semiconductors. The high exciton binding energy originates from the strong charge carrier localization and the low dielectric constant in organic semiconductor films (typically $\varepsilon \approx 3.5$ ), and thus a poorly screened Coulomb interaction of the photo-generated electron-hole pair. This results in a strongly bound Frenkel exciton, which, at temperatures and electric field strengths present in OPV devices, cannot be efficiently dissociated into charges.

a

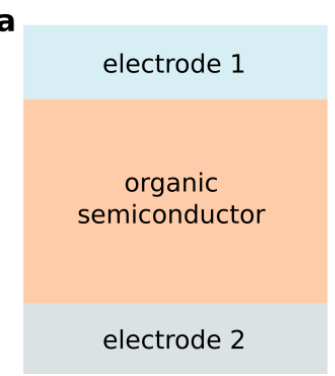

b

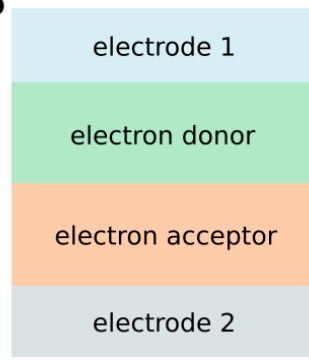

C electrode 1

donor/acceptor bulk heterojunction

electrode 2

Figure 7. (a) Single-layer, (b) bilayer and (c) bulk heterojunction organic solar cells.

The first major conceptual breakthrough came in 1986, when C. W. Tang has introduced the bilayer organic solar cell ${ }^{34}$. The top photoactive layer consisted of organic molecules with electron donating character (the donor), whereas the bottom photoactive layer was based on organic molecules with an electron accepting character (the acceptor). In this case the excitons photo-generated in either the donor or the acceptor layer diffuse to the donor/acceptor interface to be separated into charges (see Figure 8 on the next page). The difference in material electronegativity/ionization potential at the interface between the donor and the acceptor provides the necessary driving force for efficient electron/hole transfer. Following exciton dissociation, the photo-generated holes are transported to the extracting electrode via the donor, whereas the electrons are extracted via the acceptor.

The holes are transported via the Highest Occupied Molecular Orbital (HOMO) levels in the donor phase, whereas the electrons are transported via the Lowest Unoccupied Molecular Orbitals (LUMO) levels in the acceptor phase (see Figure 8 on the next page). The HOMO and LUMO levels are analogous to the valence and conductions bands in inorganic semiconductors, however, the charge transport mechanisms in organic semiconductors are in many ways different from inorganic materials. Charge transport in organic semiconductors is discussed in chapter 4. 
The introduction of the donor/acceptor interface has resulted in an orders of magnitude increase in the PCE compared to single-layer OPV devices (C. W. Tang reported PCE 1\%). However, due to the limited exciton diffusion length in organic semiconductors (typically less than $\sim 20 \mathrm{~nm}$ ) ${ }^{35}$, the total thickness of the bilayer organic solar cell is limited. Hence, an optimized bilayer absorbs little light, limiting photocurrent generation and thus the PCE of the bilayer OPV device.

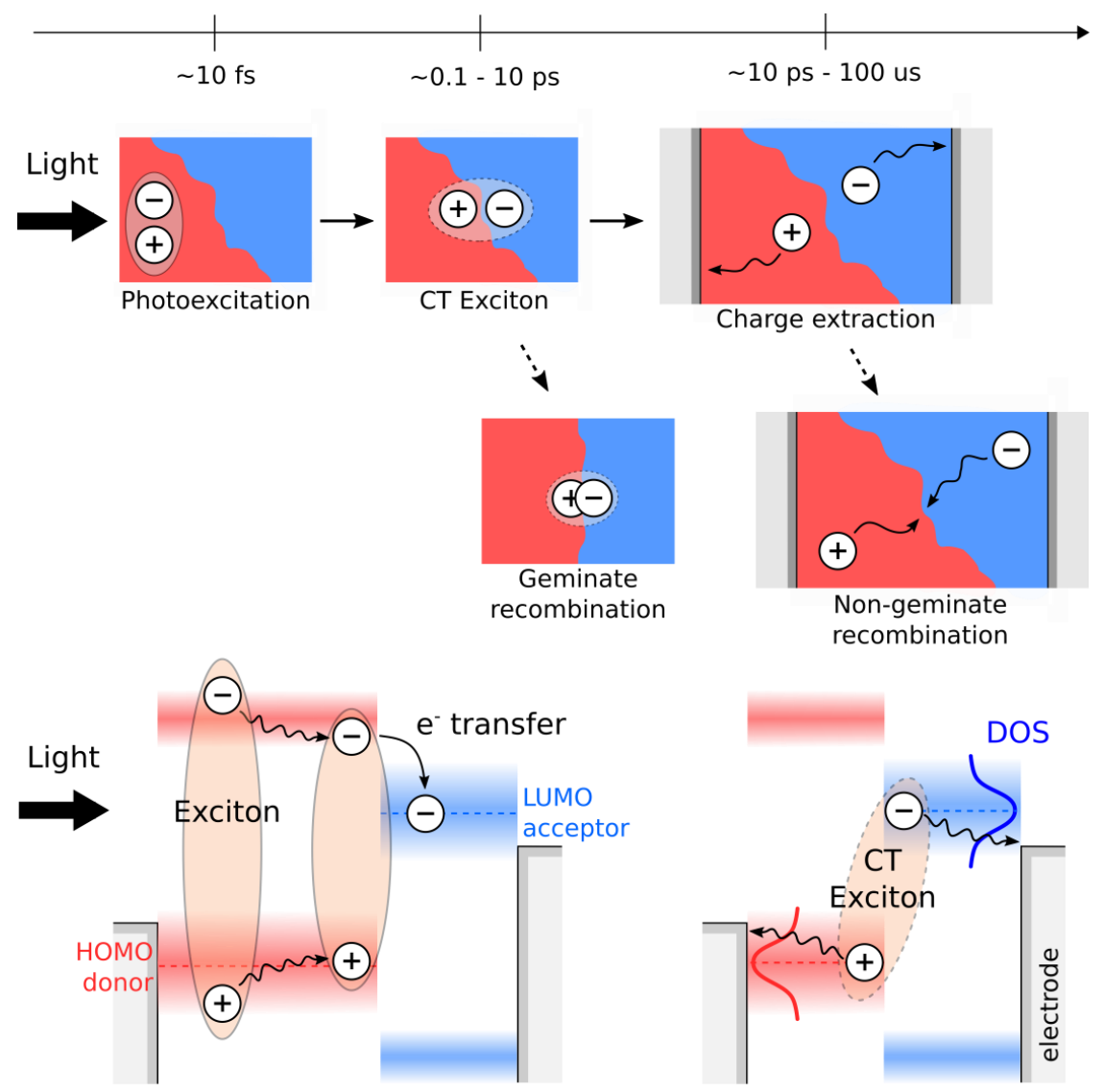

Figure 8. Schematic of the operation of a bilayer organic solar cell. The same schematic can be used to explain the operation of a bulk heterojunction organic solar cell. Although in the schematic the exciton is photo-created in the donor phase (red), similar processes take place when the exciton is photo-created in the acceptor (blue). First, exciton diffusion to the donor/acceptor interface occurs, followed by electron transfer to the acceptor. After photo-induced charge transfer, the photo-generated electron-hole pair remains Coulombically bound and temporarily forms a charge-transfer (CT) exciton. The photo-generated carriers then separate and are transported to their respective electrodes: electrons via the LUMO levels in the acceptor, holes via the HOMO levels in the donor. The HOMO and LUMO levels are broadened by energetic disorder, as indicated by the colored Gaussian Density of States (DOS). As the photo-generated carriers are transported to the electrodes, thermalization in the disorder broadened DOS occurs (black arrows in the bottom-right schematic). 


\subsection{Bulk Heterojunction}

The second major conceptual breakthrough came in 1995, when the bulk heterojunction (BHJ) organic solar cell was introduced almost simultaneously by two groups ${ }^{36,37}$. Most modern organic solar cells, including the ones studied in this thesis, are based on the $\mathrm{BHJ}$ concept. The $\mathrm{BHJ}$ consists of a disordered mixture of electron donating and accepting materials, resulting in a large number of donor/acceptor interfaces distributed throughout the bulk of the photoactive layer (Figure 7c). The donor and acceptor materials are said to form an interpenetrating donor/acceptor network.

In a $\mathrm{BHJ}$ the likelihood for an exciton to find a nearby donor/acceptor interface is generally very high. Therefore, most of the photo-created excitons reach the donor/acceptor interface and dissociate into charges. Since the entire volume of the $\mathrm{BHJ}$ contributes to exciton dissociation, the $\mathrm{BHJ}$ configuration enables the use of thicker OPV films compared to a bilayer configuration, thus absorbing more light.

However, the extraction of photo-generated charges from such a disordered $\mathrm{BHJ}$ mixture is more challenging than in a bilayer configuration. In addition, the large number of donor/acceptor interfaces promotes electron-hole recombination. The complex hierarchical arrangement of the donor and acceptor materials in the BHJ, i.e. the morphology of the photoactive layer, must be thoroughly optimized for maximum photovoltaic performance. Nevertheless, even optimized BHJs are limited to a thickness of less than a few hundred nanometers (typically $100 \mathrm{~nm}$ is used) due to charge carrier recombination and charge transport limitations. The processes taking place in a $\mathrm{BHJ}$ solar cell are summarized in Figure 8 and are discussed in more detail in the following sections.

\subsection{Exciton Dissociation}

In most OPV systems the initial photo-induced electron (or hole) transfer from the donor to the acceptor (or vice versa) occurs in less than a picosecond ${ }^{38,39}$. Although following ultrafast photo-induced charge transfer the photo-generated charges are somewhat spatially separated, they remain Coulombically bound. If the mutual Coulomb interaction is not overcome in time, electron-hole recombination commences. In this case the electron-hole pair originates from the same Frenkel exciton, the recombination of such an electron-hole pair is often termed as geminate or monomolecular (see Figure 8).

Efficient exciton dissociation occurs when the conditions at the donor/acceptor interface are favorable. This is facilitated by an appropriate choice of materials with suitable HOMO and LUMO levels. The general rule of thumb is that a driving force, i.e. a difference in the HOMO levels (for hole transfer) or the LUMO levels (for electron transfer), of $\sim 0.1-0.3 \mathrm{eV}$ is required for efficient exciton dissociation. This difference is thought to correspond to the binding energy difference between the Frenkel exciton and the charge-transfer (CT) exciton that forms following charge transfer (Figure 8). Since a fraction of the photon's energy ( 0.1-0.3 eV) must be dissipated to 
enable exciton dissociation, this reduces the amount of electrical energy that an organic solar cell can harvest per electron-hole pair. Concomitantly, the $\mathrm{V}_{\mathrm{OC}}$ of an organic solar cell is generally lower by at least $\sim 0.1-0.3 \mathrm{eV}$ compared to an inorganic solar cell with the same optical gap. New materials, enabling efficient charge separation with negligible driving force ${ }^{40,41}$, have been developed and show improved $\mathrm{V}_{\mathrm{OC}}{ }^{41}$. The future of $\mathrm{BHJ}$ organic solar cells relies on such materials.

\subsection{Charge Carrier Recombination}

In efficient OPV systems used today, geminate recombination is insignificant. Most of the initial photoexcitations rapidly generate photo-generated charges that are then transported to the electrodes ${ }^{40,42,43}$. This is also the case for the OPV systems studied in this thesis. For the time scales investigated here, photo-induced charge transfer may be considered as instantaneous. Nevertheless, the mechanism responsible for efficient charge separation on ultrafast time scales remains debated.

Following ultrafast exciton dissociation, the photo-generated charges are transported to the electrodes (Figure 8). Although electron and hole transport mostly takes place in different materials, due to the large number of donor/acceptor interfaces in the $\mathrm{BHJ}$, the likelihood for the photo-created carriers of opposite sign to meet is quite high. Upon meeting at the donor/acceptor interface, the electron and the hole form a charge-transfer (CT) exciton, which may dissociate into charges, or decay to the ground state ${ }^{44}$.

Recombination between photo-generated carriers originating from different photoexcitations is referred to as non-geminate (Figure 8). The presence of non-geminate recombination can be identified using transient techniques by following the decay in the photo-generated carrier density. For non-geminate recombination the decay is bimolecular

$$
\frac{d n(t)}{d t}=-B n_{e}(t) n_{h}(t)
$$

where $B$ is the bimolecular recombination coefficient, $n_{\mathrm{e}}(t)$ and $n_{\mathrm{h}}(t)$ are the photo-generated electron and hole densities, respectively. This is the main recombination mechanism limiting the thickness of efficient OPV devices.

Note that both the short-circuit density $\left(j_{s c}\right)$ and the open-circuit voltage $\left(V_{o c}\right)$ are limited by carrier recombination (sections 2.3 and 2.5). Therefore, carrier recombination sets the maximum attainable power conversion efficiency of the photovoltaic device. The properties of the CT state dictate both the rate of electron-hole recombination and the energetic position where recombination takes place, as discussed in the next section. 


\subsection{Charge-Transfer (CT) States}

In organic solar cells both exciton dissociation and charge carrier recombination take place at the donor/acceptor interface. In both cases the electron and the hole are situated on adjacent sites, temporarily forming a CT state. Since the properties of the CT state dictate electron-hole recombination and thus the power conversion efficiency of the organic solar cell, they are central to the overall performance of the organic solar ${ }^{31,32}$.

Due to $\mathrm{BHJ}$ disorder, differences in molecular orientations and molecular vibrations at each donor/acceptor interface, each CT state is expected to be different - the CT states are distributed in energy and are said to form a CT manifold. It turns out that CT states have distinct optical signatures and can be probed by spectroscopic tools ${ }^{45-47}$.

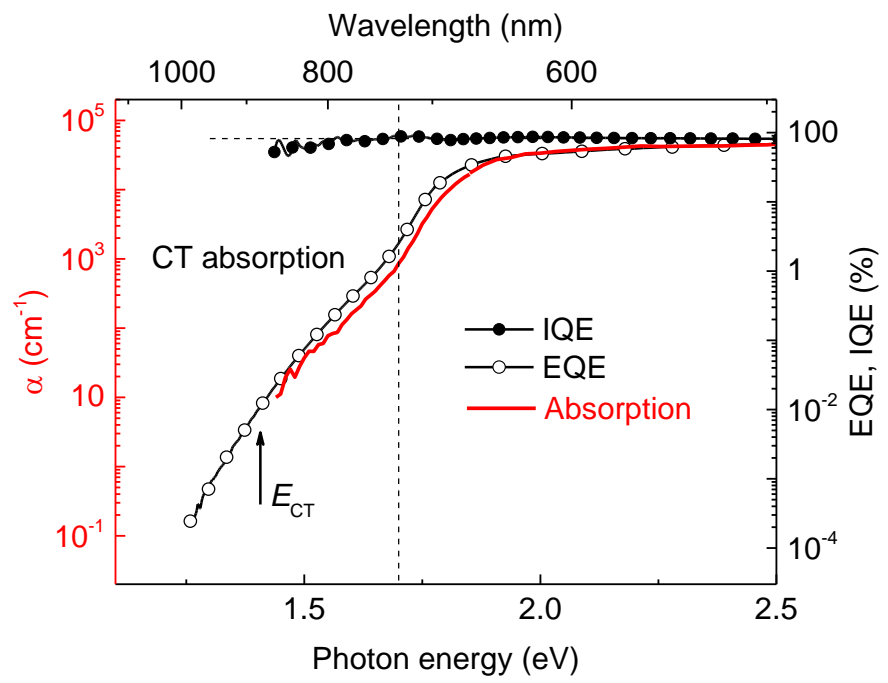

Figure 9. Sensitive EQE and absorption measurements reveal weak optical transitions in the CT manifold. Even charges photo-generated in the CT manifold are dissociated efficiently (IQE $>80 \%$ for $\left.T Q 1: P C_{71} B M\right)$. The vertical dashed line indicates the optical gap of TQ1:PC ${ }_{71} B M$.

Figure 9 shows sensitive EQE and absorption measurements on a logarithmic y-axis, where the weak optical transitions originating from absorption in the CT state manifold are visible. It turns out that efficient charge generation, i.e. CT exciton dissociation, can occur by direct excitation in the CT manifold (IQE > 80\%) even at photon energies below the optical gap of the organic solar cell (vertical dashed line). This has been shown and is generally accepted to be the case in all optimized OPV systems ${ }^{22}$. Nevertheless, absorption in the CT manifold is generally very weak and does not contribute to photocurrent generation, e.g. at $1.4 \mathrm{eV}$ only 1 photon from the incoming $10^{4}$ is converted to extracted charge. 


\subsection{Open-Circuit Voltage}

The open-circuit voltage in organic solar cells has been shown to correlate with the energetic position of the CT manifold ${ }^{31,32}$, commonly referred to as the energy of the CT state $\left(E_{C T}\right)$, marked in Figure 9. With respect to $\mathrm{V}_{\mathrm{OC}}$, the energy of the CT state plays the same role as the bandgap in inorganic solar cells ${ }^{32}$. In other words, the CT state acts as an effective bandgap ( $\left.E_{\text {eff }}\right)$ of the organic solar cell.

The difference between the optical gap and the energy of the CT state is a measure for the driving force for exciton dissociation. The optical gap of TQ1:PC ${ }_{71} \mathrm{BM}$ is $\approx 1.7 \mathrm{eV}$, whereas the energy of the $\mathrm{CT}$ state is $\approx 1.4 \mathrm{eV}$, corresponding to a driving force of $\approx 0.3 \mathrm{eV}$ in TQ1:PC $\mathrm{P}_{71} \mathrm{BM}$ (for an electron transfer from the polymer donor to the acceptor). Since the CT energy is generally lower than the optical gap, this effectively lowers the electrical energy that the organic solar cell can harvest per electron-hole pair, and hence limits $V_{\mathrm{O}}$.

The situation is quite similar to that in amorphous inorganic solar cells, e.g. amorphous silicon, where absorption by localized sites, situated below the optical gap, is similarly weak ${ }^{24,25}$. The presence of localized sites below the optical gap lowers the energy that the photovoltaic device can harvest per electron-hole pair and thus limits the open-circuit voltage ${ }^{28,29}$. The presence of weakly absorbing CT states is one of the main reasons why the open-circuit voltage in many of the organic solar cells is much lower than in inorganic solar cells with the same optical gap ${ }^{33}$.

Due to the above, the presence of a spectrally broad CT manifold is generally undesirable. EQE spectra resembling that of crystalline silicon, with a sharp onset at the optical gap of the semiconductor, would be best. The energy of the CT state would then correspond to the optical gap of the organic solar cell. Current research efforts are thus intensely focused on tailoring the properties of the CT state at the donor/acceptor interface for further improvements in open-circuit voltage ${ }^{48}$. In fact, recently reported high efficiency OPV materials do show CT energies that are almost identical to the optical gap ${ }^{41}$, significantly improving the open-circuit voltage. The next generation of high efficiency OPV materials is thus expected to have efficient exciton dissociation with a negligible driving force and a CT energy equal to the optical gap, leading to improved $V_{\text {oc. }}$ 


\section{Non-Equilibrium Charge Motion}

Following photoexcitation, the processes taking place in an organic solar cell occur at different time scales, as indicated in Figure 8 describing the $\mathrm{BHJ}$. The extraction of photo-generated charges is a dynamic process spanning a temporal range of roughly $0.1 \mathrm{ps}-10 \mu \mathrm{s}$.

In analogy to inorganic solar cells, it is generally assumed that, once exciton dissociation takes place, photo-generated carrier thermalization completes before any significant charge transport has occurred. Following ultrafast thermalization, the photo-generated carriers are then transported to the electrodes at quasi-equilibrium energies in their respective DOS. In inorganic semiconductors (without localized traps extending into the bandgap) this assumption is experimentally justified, e.g. in c-Si photo-generated carrier thermalization occurs within a time scale of less than $\sim 1$ ps (ref. 10,11). Despite lack of proof, photo-generated carrier thermalization in organic solar cells is often assumed to also occur very rapidly.

Due to the large disorder typical for organic semiconductors, this should not be the case. This is in fact well known in the literature, describing the motion of photo-created charges in disordered inorganic semiconductors, e.g. in amorphous inorganic solar cells, containing a distribution of localized trap sites extending into the semiconductor bandgap ${ }^{26,49,50}$. Photo-generated carrier thermalization to low lying localized sites occurs over significantly longer time scales. Furthermore, the gradual trapping of the photo-generated carriers to lower lying localized sites leads to a time-dependent carrier mobility, which decreases with time following photoexcitation. Due the weak intermolecular coupling and large disorder, charge transport in organic solar cells also takes places via a broad distribution of localized sites, and should therefore also occur gradually, over time scales longer than $\sim 1 \mathrm{ps}$.

Carrier thermalization in disordered organic solids has been quite extensively studied in the past, mostly by time-of-flight (TOF) mobility measurements ${ }^{12}$. Arguably the most successful theoretical model, rationalizing those findings, was developed by $\mathrm{H}$. Bässler ${ }^{12}$. The model describes charge motion in a Gaussian distribution of localized sites (as explained in the next section) and can predict the dynamics of carrier thermalization (Figure 10). However, due to the limited temporal range of TOF measurements, the model predictions are difficult to confirm over the full range of relevant time scales in OPV devices.

The development of new experimental techniques has extended the accessible temporal range quite significantly. Figure 11 shows typical carrier mobility values found in various OPV systems ${ }^{12-20,51-57}$, as estimated by the indicated experimental technique (each technique is described in the following chapters). The trend in the mobility data strongly resembles the gradual thermalization of photo-created charges, shown in Figure 10. 
The results presented in the following chapters show that charge extraction in disordered organic solar cells is completely dominated by non-equilibrium processes, governed by the gradual thermalization of the photo-generated charges. Generally speaking, the notion that carrier thermalization in OPV devices is incredibly fast, is incorrect. In fact, since thermalization can take as long as $10 \mu$ s to complete, the photo-generated charges can be extracted before reaching quasi-equilibrium.

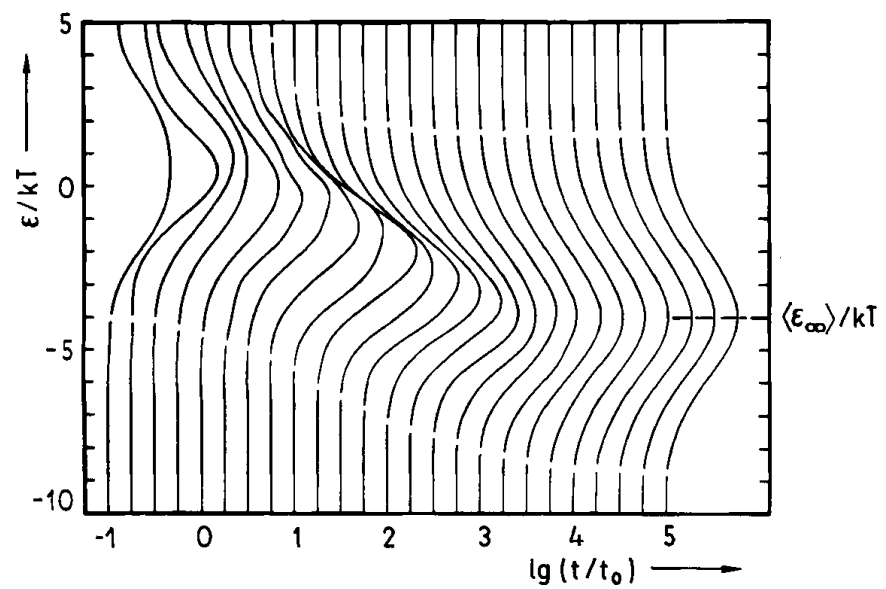

Figure 10. Photo-generated carrier thermalization in a disordered organic semiconductor, according to the Gaussian Disorder Model (GDM) developed by H. Bässler. Reproduced with permission (ref. 12).

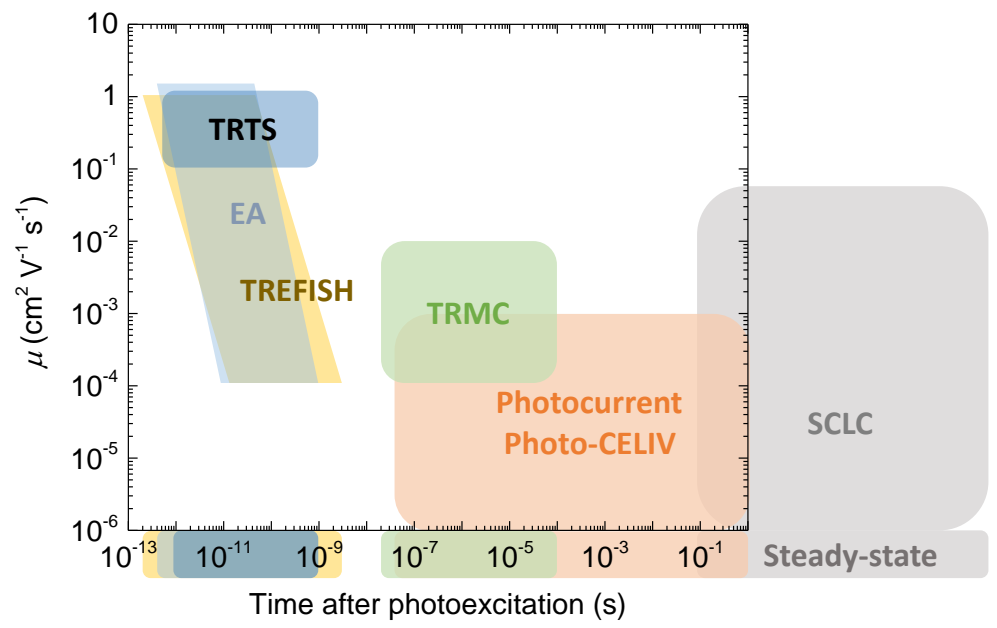

Figure 11. Schematic of the typical charge carrier mobility values in OPV, obtained by the indicated experimental technique. The indicated values not a fully exact representation of the literature data are only intended as a guide to the eye. The observed trend closely resembles the gradual thermalization of photo-generated carriers as suggested by the Gaussian Disorder Model (GDM) in Figure 10. EA stands for Electro-Absorption ${ }^{16,51}$. EA was not used in this thesis and is only shown for completion. 


\subsection{Gaussian Disorder Model (GDM)}

All of the experimental data presented in this thesis is interpreted in the framework of the Gaussian Disorder Model (GDM) ${ }^{12,58}$, which has been successfully utilized to explain charge transport in a large variety of organic semiconductors ${ }^{59-62}$. This chapter is intended as a brief introduction to the model, highlighting its main features and assumptions. Although the concepts are illustrated for a polymer film, the same reasoning can be applied to small-molecule-based films or mixtures of polymers and/or small molecules.

The model describes charge motion in a disordered energy landscape, e.g. hole transport in the polymer donor phase (Figure 12a). Chemical and physical defects, chain kinks and chain ends break the polymer chain into subunits or 'conjugated units'. These conjugated units serve as electronic sites for charge transport (Figure 12b). Due to disorder, the wavefunctions at each site are strongly localized, as first elucidated by Anderson ${ }^{63}$. This is the origin of the relatively low conductivity in organic semiconductors.

Nevertheless, charge transport between localized sites can occur by thermally activated tunneling, commonly referred to as carrier hopping (Figure 12c). The hopping rate from an initial state $i$ with energy $E_{\mathrm{i}}$ to a final state $f$ with energy $E_{\mathrm{f}}$ can be described by the Miller-Abrahams expression ${ }^{64}$, which is based on a phonon-assisted tunneling mechanism

$$
v_{i f}= \begin{cases}v_{0} \exp \left(-2 \alpha r_{i f}\right) \exp \left(-\frac{E_{f}-E_{i}}{k T}\right) & \text { if } \Delta E>0 \\ v_{0} \exp \left(-2 \alpha r_{i f}\right) & \text { if } \Delta E \leq 0\end{cases}
$$

where $v_{0}$ is the carrier attempt-to-hop frequency, generally understood to be related to some phonon frequency in the material, $r_{\text {if }}$ is the distance between the localized sites, $k$ is the Boltzmann constant, $T$ is the temperature, $\alpha$ describes the decay length of the localized wavefunctions. Typically $\alpha^{-1} \sim 0.1 \mathrm{~nm}$ is used, meaning that the charge carriers are strongly localized on a single site and that only nearest-neighbor hopping is possible. The justification of this are experimentally determined values for TNF:PVK $\left(\alpha^{-1} \approx 0.11 \mathrm{~nm}\right)^{65}$ and for P3HT and $\mathrm{OC}_{1} \mathrm{C}_{10}$-PPV $\left(\alpha^{-1} \approx 0.15 \mathrm{~nm}\right)^{59}$. In paper $V$ we show that $\alpha^{-1}=1 \mathrm{~nm}$ is also possible, enabling long-range hopping. In case of an externally applied electric field, the exponent with the sites energies $E$ also includes the electrostatic energy term.

To describe the transient experiments with the least number of unknown parameters, the inter-site distance was not explicitly accounted for in this thesis, i.e. the exponential term describing the distance dependence was implicitly included in $v_{0}$.

Generally speaking, placing a charge on a molecular site will deform the molecule and its environment. A charge in combination with the distortion of the charge's environment is called a polaron. In this thesis polaronic effects were not accounted for - carrier hopping was described by the Miller-Abrahams rate. 
The energy distribution of the localized sites through which hopping takes place is assumed to be Gaussian

$$
f\left(E_{i}\right)=\frac{1}{\sqrt{2 \pi \sigma^{2}}} \exp \left(-\frac{\left(E_{i}-E_{0}\right)^{2}}{2 \sigma^{2}}\right)
$$

Where $E_{\mathrm{i}}$ is the single particle energy on site $i, E_{0}$ is the mean energy and $\sigma$ is the standard deviation of the Gaussian DOS or simply the energetic disorder. An exponential DOS may be used instead, however, for the OPV materials studied in this thesis the Gaussian DOS was found to fit the experimental data best. In this thesis materials with energetic disorder values spanning the range $\sigma=60-140 \mathrm{meV}$, which is typical for organic semiconductors, have been investigated.

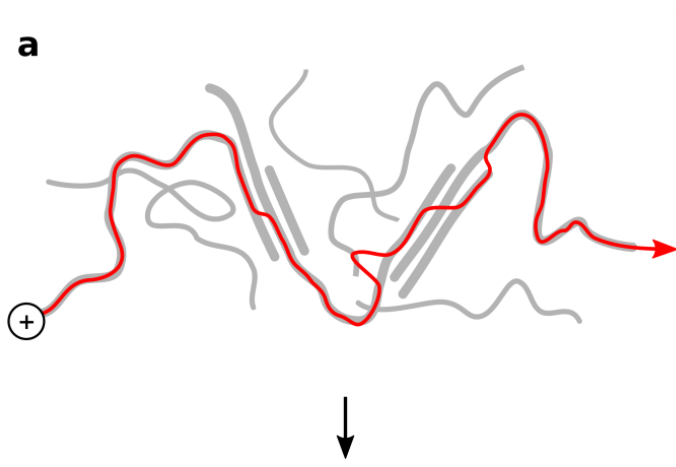

b
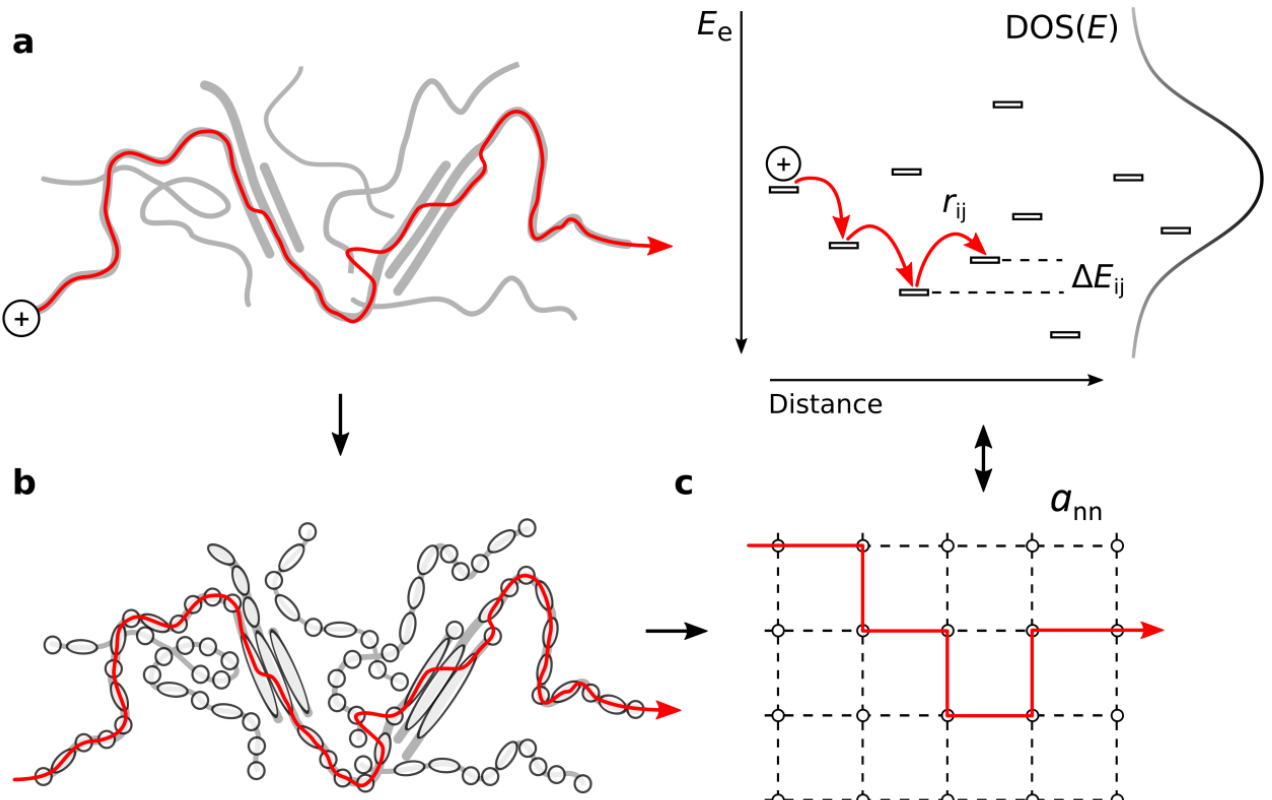

C
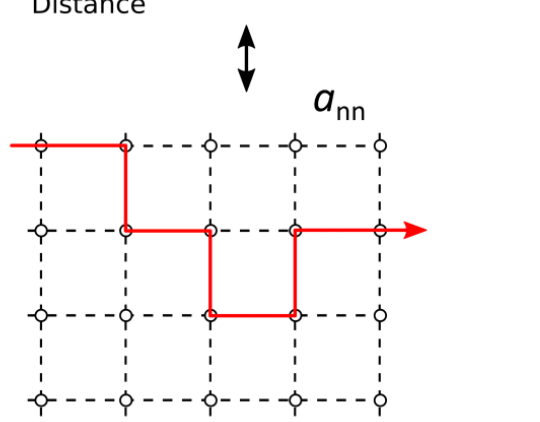

Figure 12. Schematic description of the Gaussian Disorder Model (GDM). The polymer chains in (a) can be subdivided into conjugated units or hopping sites, shown in (b), via which charge transport (red arrow) takes place. (c) The GDM model represents the electronic sites as a grid, where the site energies are drawn from a Gaussian distribution according to Equation 10. Note that the energy axis in the top-right Figure corresponds to electron energy $\left(E_{\mathrm{e}}\right)$, hole hopping in this case occurs from top to bottom.

The spatial arrangement of the polymer chains, i.e. the film morphology, determines the spatial distribution of the electronic sites. Unfortunately, such spatial distributions are not directly accessible by experiments. Hence, the GDM model relies on a further simplification. The electronic sites are instead represented as a grid with an effective inter-site distance $a_{\mathrm{nn}}$, typically in the 
range of $a_{\mathrm{nn}}=0.3-2 \mathrm{~nm}$ (ref. 60). This approach has worked remarkably well for a large number of organic semiconductors. In this thesis $a_{\mathrm{nn}}=1.8 \mathrm{~nm}$ was used, on basis of earlier work where experiments were fitted by the GDM model ${ }^{66,67}$.

Nevertheless, by mapping the multi-length scale morphology of the polymer film to a grid, morphology specific information is to a large extent lost - differences in the physical properties between disordered and ordered regions are not explicitly accounted for. Hence, the GDM parameters, such as the inter-site distance $a_{n n}$, the attempt-to-hop frequency $v_{0}$ and the energetic disorder $\sigma$, represent the average or effective values over the entire film. This approximation is most suitable for amorphous polymer films, i.e. without large semi-crystalline domains. Therefore, the main system studied in this thesis was chosen to be TQ1:PC ${ }_{71} \mathrm{BM}$ - a well inter-mixed and amorphous donor-acceptor blend ${ }^{68}$.

The GDM model is a crude description of ultrafast, and in literature suggested to be coherent ${ }^{39,69}$, phenomena occurring on a femtosecond to picosecond time scale. In addition, charge transport at the shortest time scales is expected to take place along the polymer backbone ${ }^{18}$, which is not explicitly accounted for by the GDM model. Models incorporating the spatial arrangement of the polymer chains have been proposed ${ }^{70}$. Although GDM is a rough approximation of the phenomena occurring at the earliest time scales, the main purpose of the model is not to fit a limited temporal range. The main purpose of the model is to use a single framework that describes a large number of transient experiments, spanning a wide range of times, and to accomplish this by the least number of parameters, most of which can be determined from experiments. 


\subsection{Thermalization According to the GDM framework}

Within the framework of the GDM model, photo-generated carrier thermalization takes place by carrier hopping between localized sites. This implies that carrier thermalization can only take place by hopping motion. Since on-site carrier thermalization is expected to be very fast, i.e. occur in less than a picosecond ${ }^{71,72}$, for the time scales investigated here, it can be considered as instantaneous. Only inter-site thermalization is considered in this thesis.

According to the Miller-Abrahams expression (page 23), hops that are downward in energy $(\Delta E<0)$, such as during the initial stages of carrier thermalization, occur at a considerably higher rate than upward hops. This is because downward hops are not subject to the 'Boltzmann prefactor', which reduces the hopping rate for upward hops $(\Delta E>0)$. Therefore a charge carrier initially positioned at an energy $E_{0}$ will rapidly thermalize to lower energy sites and eventually to its quasi-equilibrium energy, which is $\sigma^{2} / k T$ away from the Gaussian DOS center (in the Boltzmann limit). Paper III shows that for sufficiently thin OPV films $(100 \mathrm{~nm})$ the photo-generated carriers can be extracted from the OPV device before full thermalization takes place.

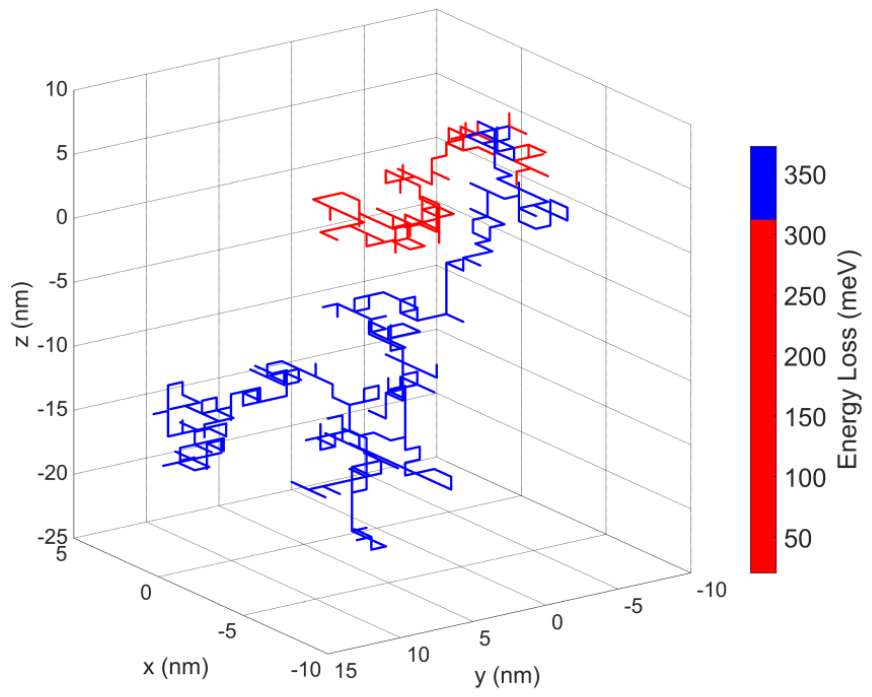

Figure 13. Simulated motion of a photo-created electron in $\mathrm{TQ1}: \mathrm{PC} \mathrm{C}_{71} \mathrm{BM}$, initially positioned at $\left[\begin{array}{ll}0 & 0\end{array}\right]$. The electric field $(0.2 \mathrm{~V} / 100 \mathrm{~nm}$, corresponding to MPP conditions) is driving the electron downwards (to negative $z$ values), however, it has little effect at early time scales ( $<1 \mathrm{~ns}$ in this case). Most of the excess photon energy is lost by fast diffusion-dominated motion (red), followed by a slower and smaller loss during drift-dominated extraction (blue). The color bar only shows two colors for clarity. 


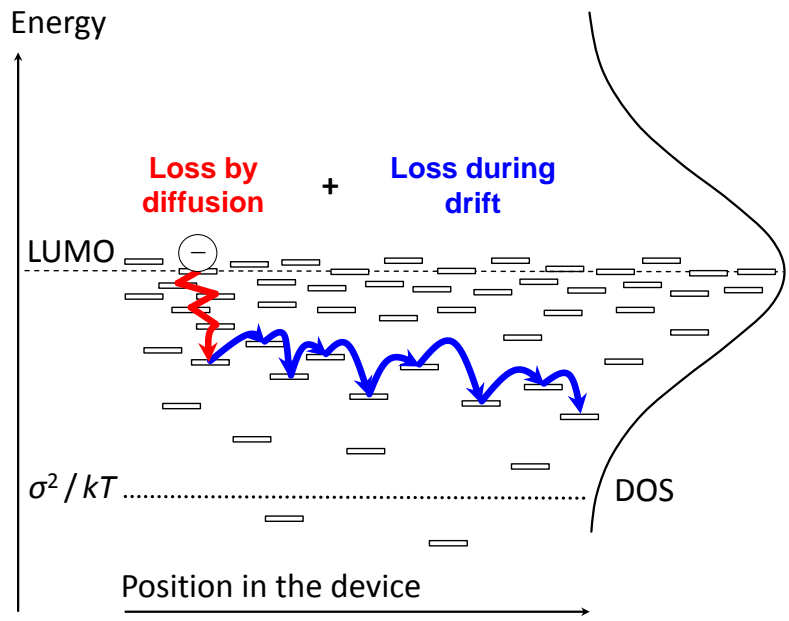

Figure 14. Schematic description of photo-generated carrier thermalization in an OPV device. First, most of the excess energy (1-2 $\sigma)$ is lost by fast diffusive motion, as indicated by the red hopping arrow going back and forth. At later time scales, the drift component of motion gradually becomes more important and directed transport to the extracting electrode begins (blue arrows). During transport to the electrode, the remainder of the excess energy is continuously, but not entirely, lost to further thermalization. The photo-generated carriers are typically extracted before reaching quasi-equilibrium at $\sigma^{2} / k T$. Adapted with permission from ref. 57.

Paper III also shows that photo-generated carrier thermalization in OPVs is a two-step process (Figures 13 and 14). At early time scales (1-100 ns) a substantial amount of energy (1-2 $\sigma)$ is dissipated by fast diffusive motion (red in Figures 13 and 14), during which most of the hops occur downward in energy ${ }^{57}$. TREFISH measurements performed by other groups have shown that the initial charge separation process on a picosecond to nanosecond time scale is indeed driven by diffusion in an energetically disordered medium ${ }^{73}$. In addition, measurements performed on neat polymer films have revealed that initial thermalization is temperature-independent ${ }^{74}$. This was rationalized by the GDM model, where carrier hopping, following the earliest time scales after photoexcitation, occurs downward in energy and does not require thermal activation. The diffusion part of the energy loss to thermalization can be minimized only by reducing the energetic disorder of the material.

In contrast, at later time scales, an increasing number of upward hops occurs and thermalization slows down considerably (blue in Figures 13 and 14). This observation is supported by earlier TREFISH measurements on neat polymer films, showing that at longer time scales following photoexcitation charge carrier motion becomes temperature-dependent ${ }^{74}$. At longer time scales an increasing number of (thermally activated) upwards hops is required for transport. In this case also, reducing the energetic disorder would minimize the losses to thermalization. 



\section{General Considerations for Time-resolved Measurements}

Non-equilibrium charge motion in OPV devices can be probed by a variety of techniques. All transient techniques share common features. Some of those features are particularly instructive and are discussed in this chapter.

Experiments probing the dynamics of photoexcited species in organic solar cell materials are typically performed either on films coated on transparent insulating substrates, or on complete photovoltaic devices, i.e. 'films with electrodes' (Figure 15a). Each approach has its own merits and drawbacks, as will be discussed below. In most cases the basic measurement scheme is: an optical pump beam is absorbed by the OPV film and creates photo-excited species, the dynamics of these species and the altered properties of the OPV film are subsequently monitored by the use of a time-delayed optical probe beam. The measurement range depends on the signal-to-noise ratio in the detected optical probe.

Transient measurements on complete photovoltaic devices, e.g. films with electrodes, are preferable. This is mostly because transient data can then be directly compared to steady-state OPV device characteristics on the same device, enabling one to check whether the transient data represents the conditions relevant for steady-state OPV device operation. In addition, the presence of the electrodes may (or may not) alter the dynamics of the photoexcited species in the film. By measuring complete OPV devices, effects due to the presence of the electrodes are included in the monitored dynamics. Electrodes also allow for bias-dependent measurements and for the detection of time-resolved electrical signals, such as transient photocurrent, providing complementary information to optical probing.

OPV devices generally have only one semi-transparent electrode that allows for the light to enter. After traversing the OPV film the probe beam is reflected at the metallic contact and can be collected by a detector in 'reflection mode'. Although this is a common measurement geometry, it is fairly limited in the sense that the OPV device can only be photoexcited and probed from the same side. In this thesis semi-transparent OPV devices ${ }^{42}$ were often employed instead, where both electrodes are semi-transparent, enabling one to change the side at which the photoexcitation or the probe beam are incident on the OPV device. An example, illustrating the advantages of using semi-transparent OPV devices, is given in chapter 6 .

Nevertheless, measurements on complete OPV devices are not always possible. For example, Time-Resolved Terahertz Spectroscopy (TRTS) or Time-Resolved Microwave Conductance (TRMC) measurements require insulating substrates, and typically quartz is used. In this case the optical probe is detected in 'transmission mode' after traversing the OPV film (Figure 15b). The advantage of not having electrodes is ease of sample preparation - the OPV film is simply coated on a suitable pre-cleaned substrate. 
However, coating the film on quartz (or glass), does not guarantee the same OPV film morphology as in the corresponding OPV device. This is because the surface energy of the quartz (or glass) substrate is likely different compared to that of the substrate used for the OPV device, possibly resulting in a different OPV film morphology. Care has to be taken.

Here, this issue was resolved by coating a monolayer of an interface material PFPA-1 (ref. 75) prior to OPV film deposition, both during OPV device fabrication and whenever OPV films were deposited on quartz or glass substrates. In almost all cases the substrate was Glass/TiOx/PFPA-1 or Glass/PFPA-1. This enabled reliable comparison of the transient data collected on films with and without electrodes.
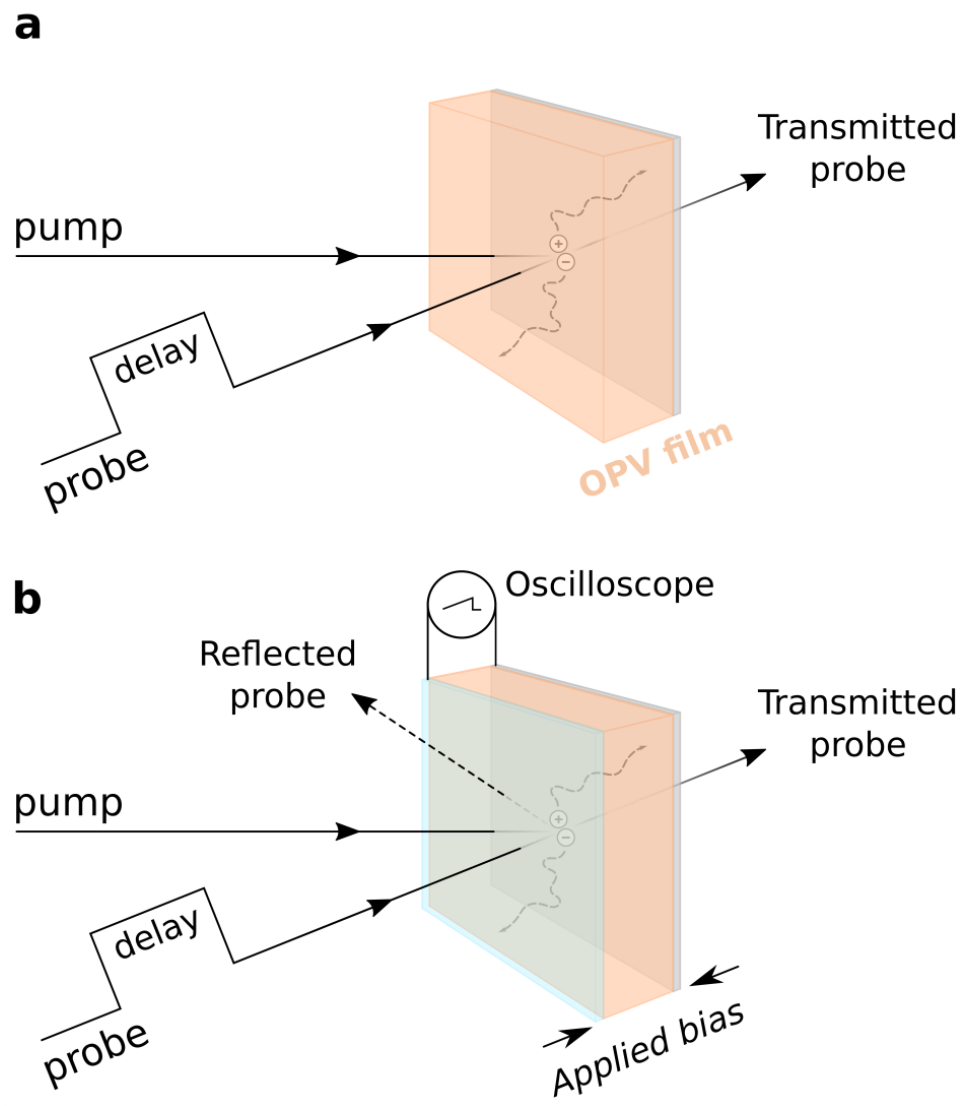

Figure 15. Schematic representation of time-resolved measurements, typically performed either on thin films (a) or on complete solar cell devices with electrodes (b). If a metallic back electrode is used, the probe is reflected (dashed arrow), whereas in the case of semi-transparent devices, the probe is transmitted (solid arrow). Electrodes enable bias-dependent measurements and the detection of time-resolved electrical signals by an oscilloscope. 


\section{Charge Extraction}

\subsection{TREFISH Combined with Transient Photocurrent Measurements}

$100 \mathrm{fs}-10 \mu \mathrm{s}$

To fully understand charge transport in operating OPV devices, the motion of photo-generated charges must be characterized at all relevant time scales - from the first hopping events to full extraction (100 fs - $10 \mu \mathrm{s}$ ). Although photo-generated charge extraction can be investigated by means of transient photocurrent measurements, RC limitations limit their use to time scales longer than nanoseconds ${ }^{76,77}$. In contrast, the first hopping events occur on a sub-picosecond to picosecond time scale. RC limitations can be avoided if the early time scales of charge motion are instead monitored by an optical probe. This can be accomplished if some optical property of the OPV film is affected by the motion of the photo-generated charges.

The OPV film sandwiched between electrodes forms a capacitor-like sample (Figure 16). Applied bias charges the plates of this capacitor, thus creating an electric field across the OPV film. If the film is then photo-excited, the motion of the photo-generated charges partially screens the electric field induced by the applied bias $E_{0}$, resulting in an electric field change $\Delta E(t)$. In this thesis $\Delta E(t)$ was measured optically by means of optical second harmonic generation.
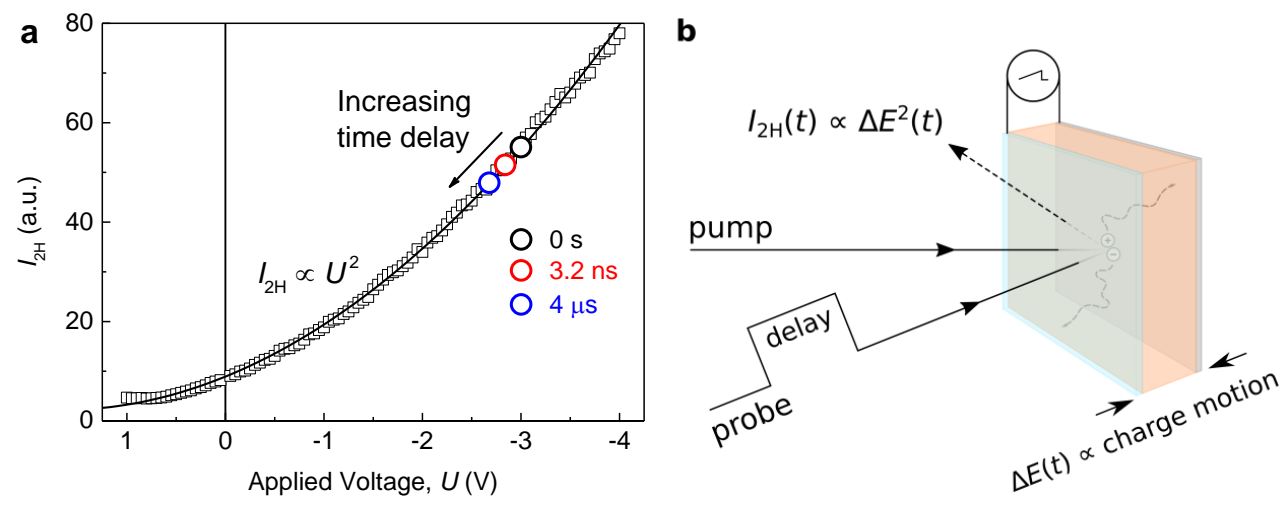

Figure 16. (a) Second harmonic intensity versus applied voltage and pump-probe time delay. (b) Scheme of the combined TREFISH and transient photocurrent measurement.

This method relies on the well-known effect of Electric-Field-Induced Second Harmonic (EFISH) generation. The second harmonic cannot be generated in amorphous or centrosymmetric materials, however, in this case the centrosymmetry is broken by the electric field. The second harmonic generation efficiency is then proportional to the second-order susceptibility of the material (or the hyperpolarizability of the molecules) and to the square of the electric field $I_{2 H} \propto E^{2}$. 
If the electric field is homogeneously distributed across the thickness of the film $d$, the applied bias $U=E d$ is an equivalent quantity, hence $I_{2 H} \propto U^{2}$ (Figure 16a). The applied bias at which the second harmonic intensity reaches its minimum value, also allows to estimate the built-in voltage of the OPV device $\left(U_{\mathrm{bi}}=1 \mathrm{~V}\right.$ in Figure $\left.16 \mathrm{a}\right)$. As such, by recording the second harmonic intensity $\left(I_{2 H}\right)$, the electric field inside the OPV film can be monitored.

The time-resolved variant of the EFISH measurement (TREFISH) is carried out in a pump-probe scheme $^{15,55}$ (Figure 16b). The pump beam creates photo-generated charges and the probe subsequently monitors the decreasing electric field strength in the OPV film. For small variations in the electric field ${ }^{15}$

$$
\Delta E(t)=\left[\left(I_{2 H}(t) / I_{2 H, 0}\right)^{1 / 2}-1\right] E_{0}
$$

where $I_{2 \mathrm{H}}(t)$ and $I_{2 \mathrm{H}, 0}$ are the second harmonic intensities with and without the pump, respectively, $E_{0}$ is the applied electric field. Small changes in the electric field are ensured by measuring at sufficiently low pump fluences, inducing electric field changes no larger than $10 \%$ of $E_{0}$ (Figure $16 a)$. The field change $\Delta E(t)$ relates to the amount of extracted charge per area as

$$
\Delta Q(t)=C d \Delta E(t)
$$

where $C$ is the sample capacitance per area, estimated by measuring the RC constant of the OPV device without photoexcitation, $d$ is the sample thickness. The amount of extracted charge per area $\Delta Q(t)$ can then be related to the extracted carrier density $\Delta n(t)$ as

$$
\Delta n(t)=\Delta Q(t) / e d
$$

where $e$ is the elementary charge. The temporal resolution of the TREFISH measurement is set by the duration of the laser pulses ( $\approx 120 \mathrm{fs}$ in this thesis), enabling sub-picosecond resolution. The temporal range of TREFISH measurements is limited by the optical delay line ( $3.2 \mathrm{~ns}$ max in this thesis). Charge extraction at time scales longer than $3.2 \mathrm{~ns}$ is monitored by a simultaneous (using the same pump pulse) recording of the photocurrent transient using an oscilloscope. As such, all relevant time scales for charge transport, from sub-picoseconds to tens of microseconds, can be probed in a single measurement (Figure 17a). A fixed pump-probe time delay also allows for field-resolved measurements (Figure 17b).

Papers II, IV and V show that the early part of these transients corresponds to electron extraction, whereas the latter part of the transients corresponds to hole extraction. Since the separate contributions of each population are difficult to disentangle using analytical methods, in this thesis kinetic Monte Carlo simulations (described in the next section), combined with complementary measurements, were employed instead.

In paper II the electron and hole extraction dynamics on an ultrafast time scale were measured separately by the use of semi-transparent OPV devices with a thick active layer ( $440 \mathrm{~nm}$ ). The idea of the measurement was inspired by conventional time-of-flight (TOF) measurements and is sketched in Figure 18. Here, the limited penetration depth of the pump and the probe beams was 32 
used to separate the signals due to the electron and hole motion. The experiment is described in the Figure caption, more details can be found in the Supporting Information of paper II.
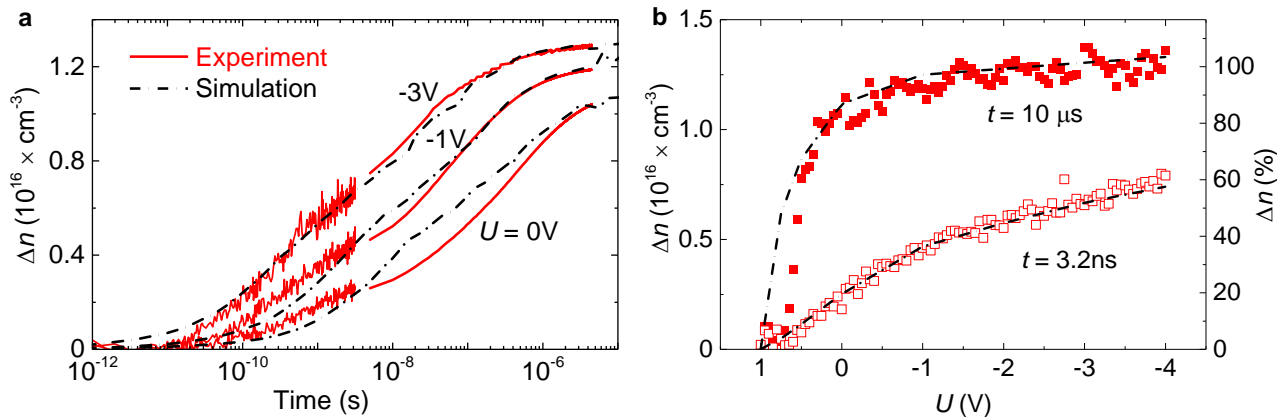

Figure 17. (a) Time-resolved and (b) field-resolved photo-generated charge extraction from a TQ1:PC ${ }_{71} \mathrm{BM}$ photovoltaic device. Red traces and symbols correspond to experiments, black traces correspond to simulations. Adapted with permission from ref. 55.

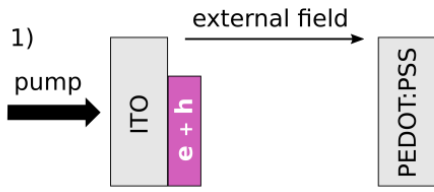

2)
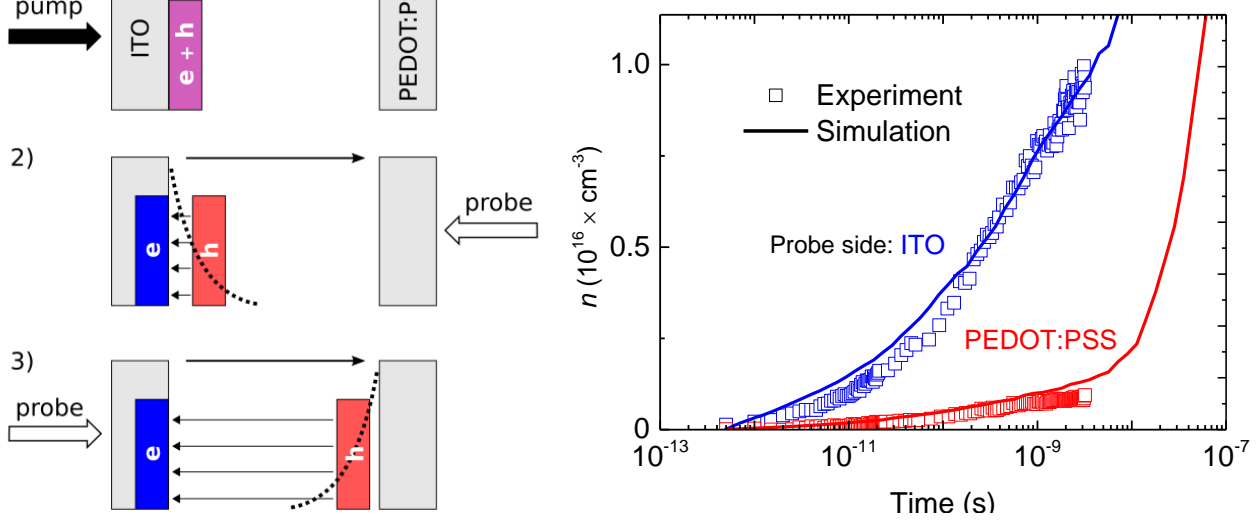

Figure 18. Left-side show a sketch of the measurement. (1) The pump creates a relatively narrow distribution of photo-generated charges (purple rectangle). (2) Only when the region where the field is present, i.e. between the sheets of electrons (blue) an holes (red), extends to the area 'seen' by the probe (dotted lines) a TREFISH signal is measured. The area 'seen' by the probe is on the opposite side of the sample with respect to the incident probe. This is because the second harmonic is reabsorbed by the active layer and only the indicated fraction (dotted lines) can exit the device for detection. Since holes drift much slower than electrons and need to traverse a larger distance, the TREFISH signal at the ITO electrode is seen at short times (2), whereas the signal at the PEDOT:PSS electrode is delayed (3). Right-side shows that the measurement results of the sketched experiment are in good agreement with kinetic Monte Carlo simulations. Adapted with permission from ref. 55. 


\subsection{Simulations using the Gaussian Disorder Model}

The combined TREFISH and photocurrent measurement fully spans the relevant time scales for charge motion, i.e. from the first hopping events, occurring on a sub-picosecond time scale, to full extraction at tens of microseconds. Such experimental data is thus ideally suited for testing charge transport models. Furthermore, any model that attempts to fit the data must also reproduce the field-dependence observed in experiments, severely constraining the model. In this thesis all of the experimental data were successfully fitted by kinetic Monte Carlo simulations based on the GDM model. The implementation of the model is described in detail in the Supplementary Information of paper III.

The kinetic Monte Carlo algorithm does not contain implicit assumptions of quasi-equilibrium. Instead, the photo-generated charge carrier populations are allowed to thermalize as set by the carrier hopping parameters. By fitting the model to the experimental TREFISH and photocurrent data, statements on whether the photo-generated carriers have (or have not) reached quasi-equilibrium during their presence in the OPV device can be made.

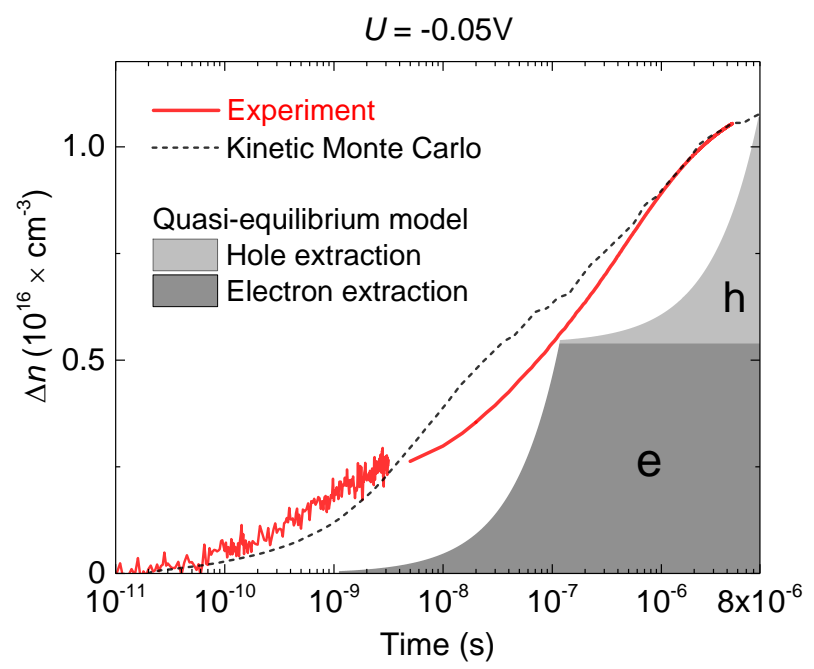

Figure 19. (a) Quasi-equilibrium model based on a single mobility value (shaded areas) cannot explain the combined TREFISH and photocurrent experiment (red trace), whereas kinetic Monte Carlo simulations allow for a reasonably good fit (black dashed trace). Data for TQ1:PC $C_{71} B M$ at close to short-circuit conditions $(U=-0.05 \mathrm{~V})$. Adapted with permission from ref. 55.

Figure 19 shows reasonably good agreement between the experiment and kinetic Monte Carlo simulations. In contrast, the shaded areas indicate the outcome if a simplified model was used. The simplified model is based on experimentally measured quasi-equilibrium mobility values using a single technique (Photo-CELIV, page 51), which probes the mobility of fully thermalized charge carriers. The main assumptions of the simplified model are: 1) all of the photo-generated charge 
carriers have an identical mobility. 2) The mobility of the photo-generated charge carriers is time-independent as they are in quasi-equilibrium. Since the simplified model captures neither the rapid extraction of the photo-generated charges nor the shape of the experimental extraction kinetics, the notions of the simplified model are false.

Instead, paper II shows that due to the stochastic nature of carrier hopping in disordered media, the photo-generated charge population is instead characterized by a wide distribution of extraction times, i.e. charge transport is dispersive. Furthermore, the mobility of the photo-generated charges turns out to be time-dependent due to their gradual thermalization in the disorder broadened DOS. Both of these aspects are discussed in more detail in the following sections.

\subsection{Dispersion in Extraction Times}

Since carrier hopping between localized sites is stochastic, i.e. random process, even charges traversing the same distance will be extracted at different times following photoexcitation. As the experimental trace represents the integral of the charge extraction distributions, by taking a log-derivative of the experimental trace, the distribution of extraction times can be obtained (Figure 20).

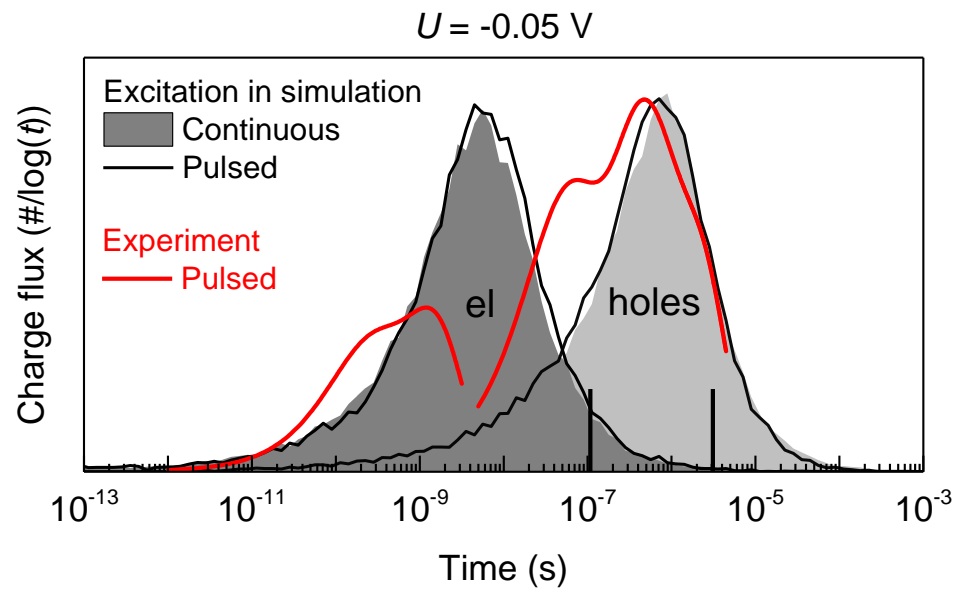

Figure 20. Dispersion in extraction times at close to short-circuit conditions $(U=-0.05 \mathrm{~V})$ in a TQ1:PC $C_{71} \mathrm{BM}$ device. According to our simulations (black traces and shaded areas), the distribution in charge extraction times in OPV devices operating under continuous illumination conditions (shaded areas) can be estimated by pulsed laser measurements (black traces). The red trace corresponds to the extraction time histogram determined from smoothed experimental data in Figure 19. Adapted with permission from ref. 55 .

Since charges in an OPV device are photo-generated at a different distance from the extracting electrode, the broadening in Figure 20 is at least in part due to a different transport distance. 
However, this broadens the extraction time distribution only by $\sim 2$ orders of magnitude in time, as can be inspected from the quasi-equilibrium model in Figure 19, where the first electrons are extracted at $1 \mathrm{~ns}$, whereas the last electron is extracted at $100 \mathrm{~ns}$. In contrast, the broadening in Figure 20 spans 5-6 orders of magnitude in time and is due to the dispersive nature of charge transport in disordered media.

The wide distribution in extraction times reveals several key features regarding photocurrent in OPV devices. First of all, kinetic Monte Carlo simulations suggest that the charge extraction distributions obtained by pulsed laser excitation are identical to those obtained under continuous illumination (Figure 20). Therefore, the distribution of extraction times in OPV devices operating under continuous 1 sun illumination conditions can be determined by pulsed laser measurements (using sufficiently low pump-fluences), as described in paper II.

Due to dispersion, mean extraction times are a particularly bad measure of the actual charge extraction in OPV devices. The vertical black bars in Figure 20 indicate the mean extraction time for the holes and for the electrons. At the mean extraction time about $80-90 \%$ of all the holes/electrons have been extracted already. In addition, by inspecting the charge extraction histograms it is clear that $50 \%$ of the holes/electrons (the early time part of the histogram, to the left of the peak maximum) are extracted orders of magnitude faster than the remaining $50 \%$. This illustrates that even a well-defined mean mobility, would be limited in view of the large dispersion.

Most of the experimental techniques that estimate the charge carrier mobility are largely insensitive to dispersion and provide an estimate only for the mean mobility of the corresponding charge carrier population. In view of the strong focus in this thesis to confirm simulations by experiments, only the mean mobility is discussed in the rest of the text. However, the associated spread around this mean must be considered when analyzing the data. 


\subsection{Time-dependent Carrier Mobility}

In addition to dispersion in extraction times, the quasi-equilibrium model also underestimates the rapid extraction of the photo-generated charges (Figure 19). Kinetic Monte Carlo simulations suggest that on the time scales of the TREFISH and photocurrent measurement the photo-generated charge carriers undergo a gradual transition from non-equilibrium to quasi-equilibrium charge motion. As such, the experimental extraction kinetics are governed by the gradual thermalization of the photo-generated carriers in the disorder broadened DOS (Figure 21). Paper III shows that most of the photo-generated charges are extracted from the OPV device before reaching quasi-equilibrium.

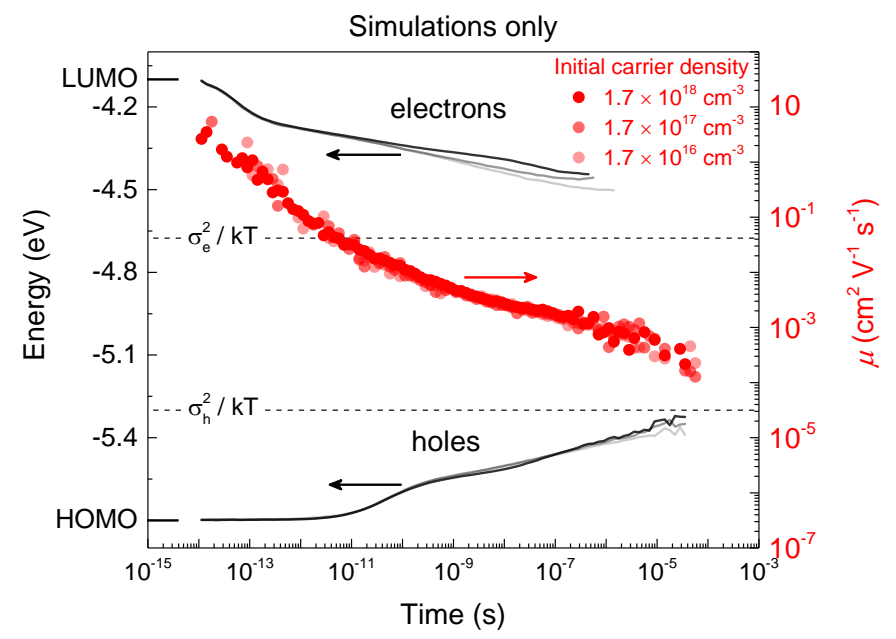

Figure 21. Simulations fitting the transient data in Figure 19 reveal that the photo-generated carriers undergo gradual thermalization in their respective DOS (black traces), resulting in a time-dependent carrier mobility (red symbols). The simulated mobility corresponds to the average mobility of electrons and holes $\mu(t)=\left[\mu_{\mathrm{e}}(t)+\mu_{\mathrm{h}}(t)\right] / 2$. Adapted with permission from ref. 55 .

The gradual thermalization of photo-created charges via localized sites leads to a time-dependent carrier mobility (Figure 21). This can be rationalized by the GDM model - the mobility of the photo-generated charges decreases with time as an increasing number of upward hops is required for charge transport at later time scales following photoexcitation. Simulated thermalization dynamics are independent of the initial density of photo-created charges as, when out-of-equilibrium, concentration effects related to DOS state-filling are considerably less important. This observation is explained in more detail in section 7.3.

Although the combined TREFISH and photocurrent measurements discussed so far are indicative of the gradual thermalization of photo-created charges, the confirmation that these processes take place has thus far been largely based on simulations. The remainder of this thesis provides complementary experimental evidence, confirming that charge extraction in disordered OPVs is indeed dominated by non-equilibrium effects. 



\section{Thermalization}

\subsection{Overview of Time-Resolved Measurement Results}

In this chapter experimental evidence supporting the notion that photo-generated carriers undergo gradual thermalization in energetically disordered OPV systems is presented. To support this conclusion all of the relevant time scales for charge motion in OPVs, from sub-picoseconds to tens of microseconds, must be investigated. As most time-resolved techniques probe the dynamics of photo-excited species only over a limited temporal range (providing only a snapshot of the full dynamics), to get a consistent physical picture, several techniques must be employed.

An overview of the collected results is shown in Figure 22, confirming that charge extraction in disordered OPVs, as revealed by TREFISH and photocurrent experiments in chapter 6 (paper II), is indeed governed by the gradual thermalization of photo-created charges.
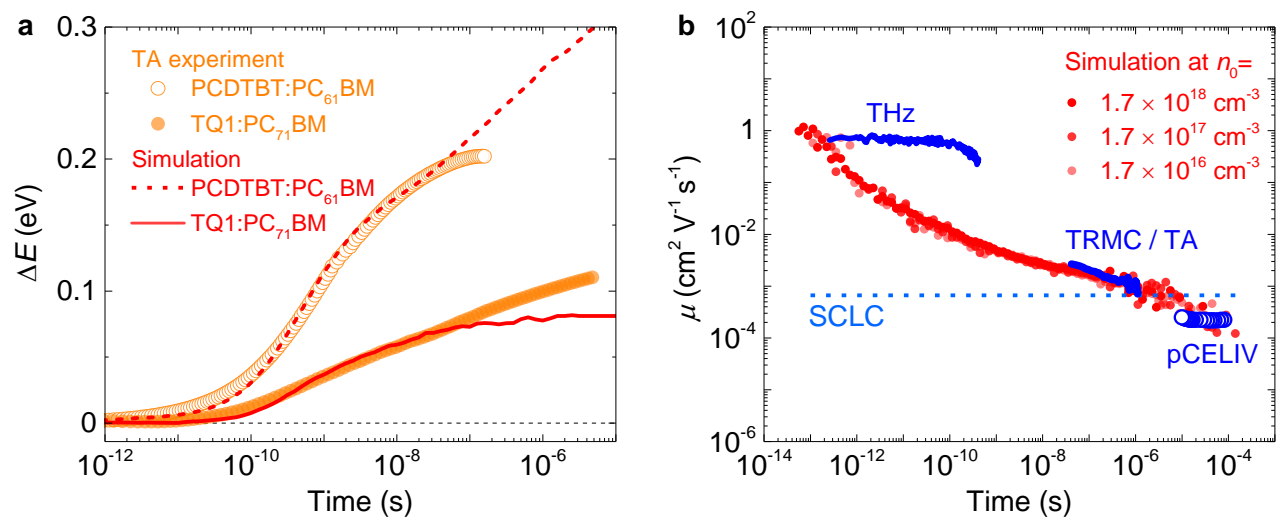

Figure 22. (a) Hole thermalization dynamics measured by transient absorption (TA) spectroscopy (orange symbols) and simulated by kinetic Monte Carlo (red traces). (b) Time-dependent carrier mobility as measured by the indicated opto-electrical technique (blue), steady-state electron mobility as measured by Space-Charge Limited Currents (SCLC) (dashed blue line) and the carrier mobility obtained by kinetic Monte Carlo (red symbols). $n_{0}$ is the initial carrier density. Adapted from ref. 57 .

Paper III confirms that although the different transient techniques probe different time scales, they are mutually consistent, as they monitor the same physical mechanism governing charge motion - charge carrier thermalization in a disorder broadened DOS. In paper IV the results have been extended to polymer/polymer blends and steady-state measurements not involving photons (SCLC), whereas in paper $V$ the results have been generalized by investigating small-molecule-based OPV blends. This thesis demonstrates that a unification of the commonly employed opto-electric techniques that probe charge motion in disordered organic semiconductors is possible. Each technique is presented in the sections that follow. 


\subsection{Transient Absorption (TA) Spectroscopy}

$100 \mathrm{fs}-10 \mu \mathrm{s}$

Pump-probe Transient Absorption (TA) spectroscopy allows to monitor the dynamics of photo-excited species over an exceptionally wide temporal range (100 fs $-10 \mu \mathrm{s})$. TA provides information regarding the dynamic processes of excited-state energy migration, photo-induced charge transfer and charge carrier recombination. In addition, measurements can be performed either on thin films or complete OPV devices. This technique is widely used for the investigation of non-equilibrium processes in OPVs ${ }^{39,78-80}$. In this thesis TA measurements were performed to measure the photo-generated carrier density $n(t)$ and hole thermalization $\Delta E(t)$ dynamics.

In TA measurements a pump beam creates photo-excited species. After a time delay $t$, a low intensity probe beam (that does not significantly affect the excited-state population) traverses the sample and is recorded by a detector. Using broadband (white light supercontinuum) probe pulses, the transmission spectra over a range of wavelengths can be recorded simultaneously, i.e. for every single shot of the probe pulse. A difference in the transmission spectra is then calculated, i.e. the transmission spectrum of the photo-excited sample minus the transmission spectrum of the sample in the ground state. By varying the time delay between the pump and the probe a time-resolved difference spectrum $\Delta T(t, \lambda)$ is recorded (Figure 23).

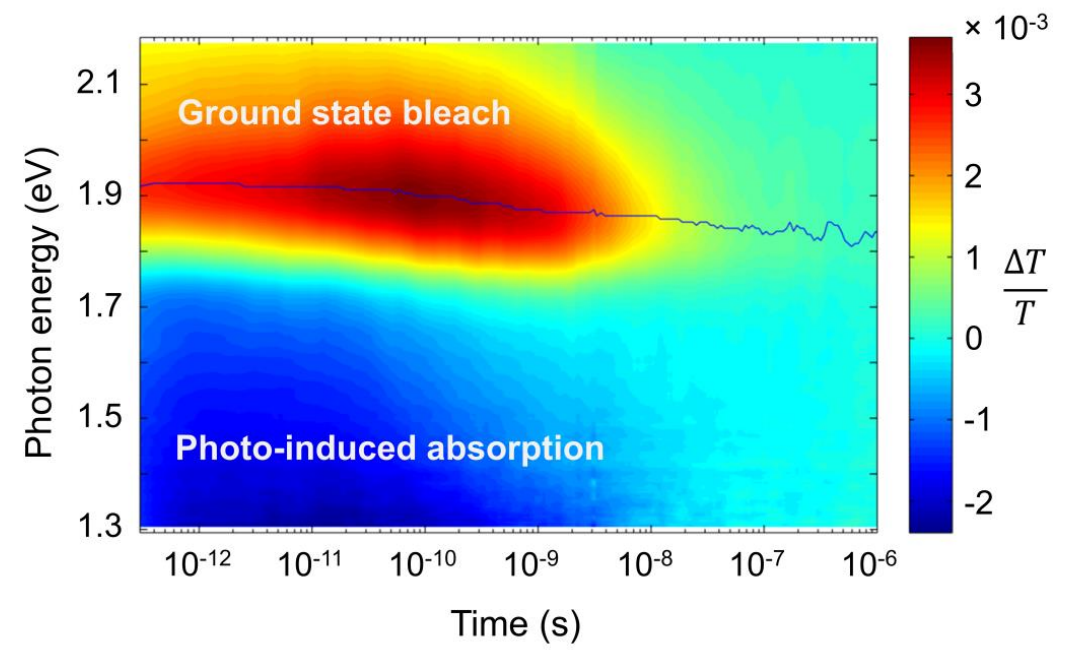

Figure 23. TA dynamics of a TQ1:PC $C_{71} \mathrm{BM}$ film photo-excited by a $2.33 \mathrm{eV}(532 \mathrm{~nm})$ pump at a pump-fluence of $3.5 \times 10^{13}$ photons $\mathrm{cm}^{-2}$ per pulse and probed by a supercontinuum (white light) probe. The redshift of the Ground State Bleach (GSB) peak maximum is indicated by the dark blue trace. Adapted from ref. 57. 
Generally speaking, the interpretation of TA data is quite complicated as multiple processes and photo-excited species contribute to the $\Delta T$ spectrum

$$
\frac{\Delta T(t, \lambda)}{T(\lambda)}=-\sum_{i, f} \sigma_{i f}(\lambda) \Delta n_{i}(t) d
$$

where $\sigma_{\text {if }}$ is the absorption cross-section for an optical transition from an initial state $i$ to a final state $f, \Delta n_{i}(t)$ is the population density change induced by the pump, $d$ is the sample thickness and $T(\lambda)$ is the sample transmission without the pump excitation. The absorption cross-section is in this case defined as negative for upward transitions and positive for downward transitions.

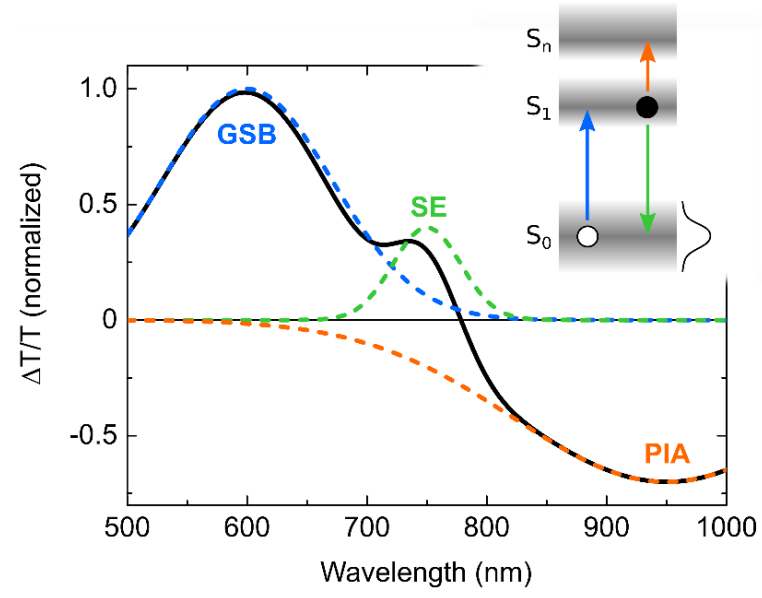

Figure 24. Schematic illustration of the different processes contributing to the TA spectrum. The inset shows the corresponding optical transitions, which are broadened due to energetic disorder.

The contributions from various processes to the TA spectrum (Figure 24) are:

- Ground State Bleach (GSB). As a fraction of the molecules (or chromophores in the polymer) have been promoted to an excited state by the pump pulse, the fraction of the molecules remaining in the ground state has decreased. Therefore, the ground state absorption in the photo-excited sample is less than in the non-excited sample, i.e. the sample has been temporarily 'bleached'. This results in a positive contribution to the $\Delta T / T$ spectra in the spectral range of ground state absorption.

- Stimulated Emission (SE). Since a fraction of the molecules have been promoted to an excited state, a probe beam at a resonant wavelength may induce emission, usually referred to as stimulated emission. As the photons originating from stimulated emission are emitted in the same direction as the probe beam, this will result in a positive contribution to the $\Delta T / T$ spectrum. The SE spectrum resembles the fluorescence spectrum of the excited molecule and is redshifted, i.e. Stokes shifted, with respect to the GSB. 
- Photo-Induced Absorption (PIA). The photo-excited species may also contribute to the $\Delta T / T$ spectrum by allowed optical transitions to higher excited states. PIA features thus indicate the presence of photo-excited species. Generally, in OPV materials, contributions from PIA span a wide spectral range, typically extending far into the near-infrared (NIR) range. If several photo-excited species coexist simultaneously, multiple, often overlapping, spectral features may be observed. Advanced (global analysis) techniques are then required to disentangle their individual contributions to the full $\Delta T / T$ spectrum.

\section{TA data interpretation}

In fullerene acceptor based OPV blends, the photo-induced optical signatures originating from the fullerenes are generally significantly weaker (due to their significantly lower absorption cross-sections) compared to those originating from the donor polymer. As such, the $\Delta T / T$ spectra are typically dominated by optical signatures originating from the donor polymer. This simplifies the interpretation of TA data.

The broad PIA feature $(<1.75 \mathrm{eV})$ visible in Figure 23 was assigned to the hole polaron ${ }^{81}$. The hundreds of femtoseconds (or less) rise in the PIA feature (Figure 23, blue feature) indicates that in TQ1:PC $C_{71} B M$ the formation of charge-transfer states and photo-generated carriers occurs on a time scale of hundreds of femtoseconds or less, i.e. almost instantaneously, as expected in efficient OPV blends. By measuring the subsequent decay of the PIA feature over a range of pump-fluences, the dominant charge carrier recombination mechanisms can be determined. For TQ1:PC ${ }_{71} B M$ the transient PIA decay on a nanosecond to tens of microseconds time scale is bimolecular (Figure 25, left y-axis) - photo-generated charges recombine non-geminately.

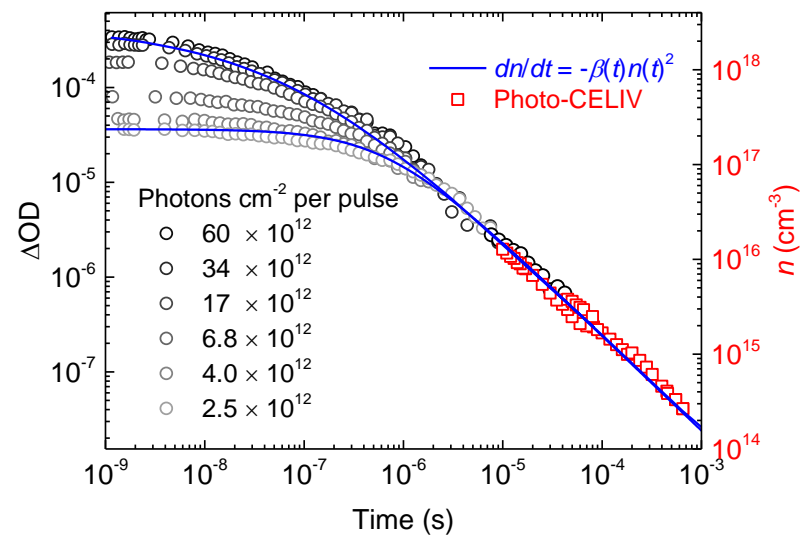

Figure 25. Combined transient absorption (black symbols, left $y$-axis) and electrical extraction (red symbols, right $\mathrm{y}$-axis) measurements on a TQ1: $\mathrm{PC}_{71} \mathrm{BM}$ device biased at 'flat-band' conditions. Carrier recombination dynamics fit best to a bimolecular decay with a time-dependent rate $\beta(t)$ (blue trace). The unit on the left $y$-axis corresponds to a change in optical density, commonly used instead of $\Delta T / T$. Adapted with permission from ref. 81. 
Figure 25 data were collected on an OPV device connected to a function generator and an oscilloscope. The OPV device was positively biased throughout the measurement to facilitate close to 'flat-band' conditions. As such, the measured carrier density decay dynamics in Figure 25 are also indicative of what occurs in an OPV film without electrodes.

To calibrate the PIA signal intensity to the more useful quantity of photo-generated carrier density $n(t)$, TA measurements were complemented by a simultaneous (using the same pump pulse) electrical recording of the amount of extracted charge using an oscilloscope (Figure 25, right $y$-axis). Electrical extraction data is available only for time scales longer than $10 \mu$ s due to experimental limitations. The data overlap in the tens of microseconds time scale enables the conversion of PIA signal intensity to photo-generated carrier density (paper I). These kinetics were later used to convert the time-resolved conductivity $\Delta \sigma(t)$, as probed by TRMC, to charge carrier mobility $\mu(t)=\Delta \sigma(t) / e n(t)$ on the 20 ns to $1 \mu$ s time scale (section 7.3).

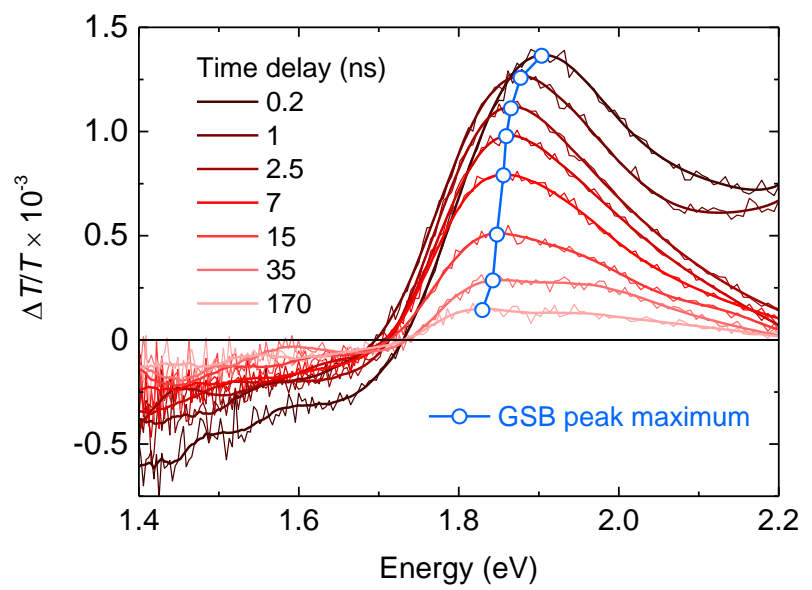

Figure 26. Slices of the time-resolved dataset, shown in Figure 23. The spectral redshift of the GSB peak maximum is highlighted by the blue trace, see Figure 22a for a plot of GSB redshift vs. time.

The spectral broadening of the GSB and PIA absorption features in Figure 23 is at least in part due to the disorder broadened DOS of the donor polymer, as schematically illustrated in Figure 24 . Since the GSB feature is due to the presence of a hole polaron on the polymer, the spectral position of the GSB peak maximum is indicative of the hole energy in the DOS. Hence, temporal redshifts of the GSB peak maximum can be used as a measure for hole thermalization. This was used to measure the hole thermalization dynamics in paper III.

The observed gradual redshift of the GSB peak maximum in TQ1:PC ${ }_{71} \mathrm{BM}$ is highlighted by the dark blue trace in Figure 23 and is also shown in Figure 26. In paper III the thermalization dynamics of photo-induced holes were estimated for two material systems, showing a total GSB redshift of $\approx 0.1 \mathrm{eV}$ in TQ1:PC ${ }_{71} \mathrm{BM}$ and $\approx 0.2 \mathrm{eV}$ in $\mathrm{PCDTBT}: \mathrm{PC}_{61} \mathrm{BM}$. The larger redshift in $\mathrm{PCDTBT:PC_{61 } B M}$ was shown to be due to the larger energetic disorder of PCDTBT ${ }^{82}$. 


\subsection{Time-Resolved Terahertz Spectroscopy (TRTS)}

$1-100 \mathrm{ps}$

The use of $\mathrm{THz}$ radiation as a probe allows to monitor the charge carrier mobility dynamics at the earliest time scales following photo-generation ${ }^{83}$. For OPV materials the measurement range typically spans $1-1000 \mathrm{ps}$, with the long time limit set by the signal-to-noise ratio. This temporal window is particularly important for the investigation of photo-induced charge separation at the donor-acceptor interface ${ }^{72}$, followed by charge transport away from the interface. In this thesis TRTS measurements were used to estimate the carrier mobility at picosecond time scales.

The duration of a THz probe pulse incident on the sample is of the order of $1 \mathrm{ps}$, corresponding to a frequency of $1 \mathrm{THz}$ (photon energy $=4.1 \mathrm{meV}$ ). Mobile charged species absorb at such low photon energies, which makes $\mathrm{THz}$ pulses probes of conduction. $\mathrm{THz}$ pulses interact strongly with conductive layers, e.g. electrodes. As a result, measurements on OPV devices are not possible. Spin-coated or drop-casted OPV films on quartz substrates are used instead. An example of a $\mathrm{THz}$ pulse, which was recorded after traversing a typical OPV film (regioregular-P3HT:PC 61 BM) coated on a quartz substrate is shown in Figure 27 (grey line, no photoexcitation).

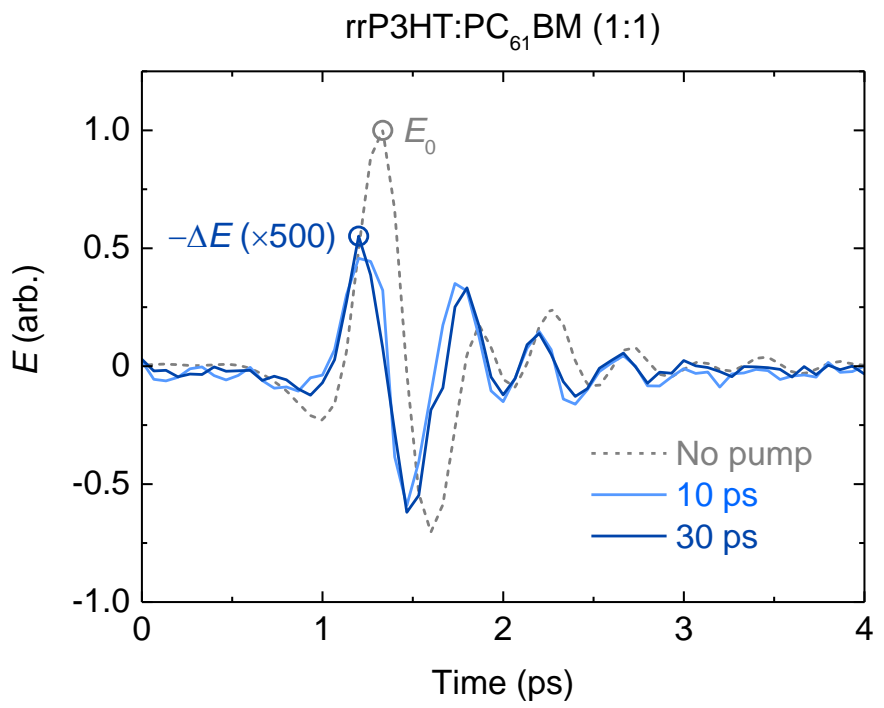

Figure 27. $\mathrm{THz}$ waveform recorded after traversing a regioregular- $\mathrm{P} 3 \mathrm{HT}: \mathrm{PC}_{61} \mathrm{BM}$ ( $\mathrm{rrP} 3 \mathrm{HT}: \mathrm{PC}_{61} \mathrm{BM}$ ) film without photoexcitation (dashed grey trace) and the relative change in the $\mathrm{THz}$ waveform at the indicated pump-probe time delay (blue traces).

In TRTS measurements an optical pump pulse is used to photo-generate charge carriers. The photo-conductance of the OPV film is subsequently monitored by a time-delayed THz probe pulse. The difference in the transmitted $\mathrm{THz}$ waveforms with and without the pump (Figure 27 blue traces) is a measure of the photo-induced conductivity in the time-domain. 
For the investigation of transient photo-conductivity in OPV blends, typically only the peak amplitude of this difference, labelled as $\Delta E$ in Fig. 26 (blue round symbol), is monitored. This assumes that the temporal evolution of $\Delta E(t)$ is mainly due to amplitude modulation of the peak maximum of the $\mathrm{THz}$ pulse and not due any temporal shifts of the waveform mimicking amplitude modulation. Although the most accurate way is to record the entire $\Delta E(t)$ waveform at each pump-probe time delay, this would demand very long measurements. Therefore, generally only the peak of the $\mathrm{THz}$ waveform is monitored, enabling a simpler and faster mode of data collection.

The measured relative change in the transmitted $\mathrm{THz}$ pulse amplitude $\Delta E(t) / E_{0}$, where $E_{0}$ corresponds to a reference measurement without the pump (see Figure 27), can be related to photo-induced conductivity using thin-film optics, commonly referred to as the Tinkham formula ${ }^{84}$

$$
\Delta \sigma(t)=\frac{1+n}{Z_{0} d}\left(\frac{-\Delta E(t) / E_{0}}{1+\Delta E(t) / E_{0}}\right)=e \mu(t) n(t)
$$

where $Z_{0}$ is the impedance of free space $(377 \Omega), n$ is the refractive index of the quartz substrate $(n=2), e$ is the elementary charge and $d$ is the sample thickness. The transient mobility of the species $\mu(t)$ giving rise to the observed photo-conductivity signal can be estimated, if their number density $n(t)$ is known. An estimate for $n(t)$ can be obtained by a complementary TA measurement performed on the same film at identical pump excitation conditions.

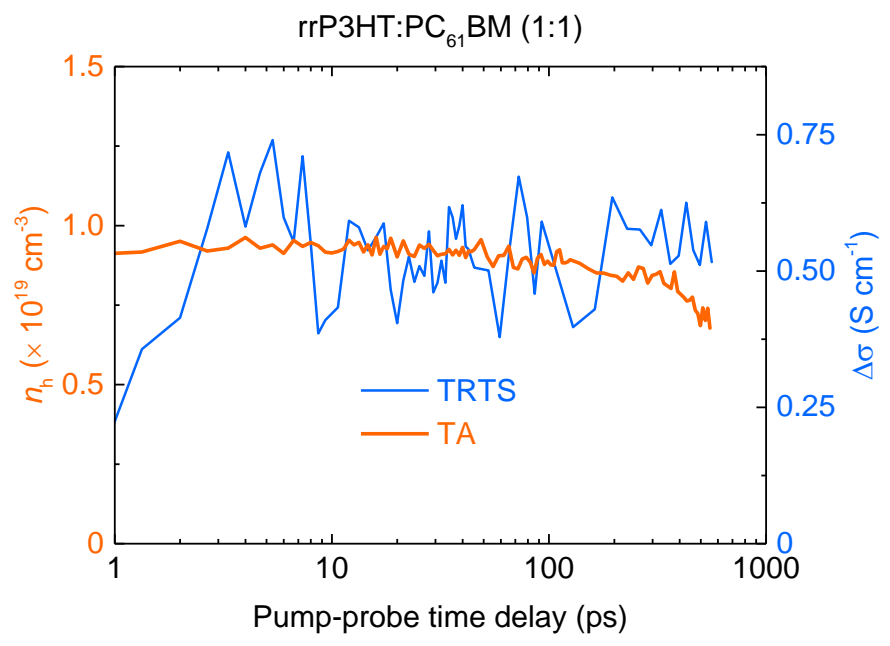

Figure 28. Comparison of the hole polaron density, as determined by TA (orange trace), and TRTS photo-conductivity dynamics (blue trace) recorded on the same $\operatorname{rP} 3 \mathrm{HT}: \mathrm{PC}_{61} \mathrm{BM}$ film at an identical excitation fluence $\left(120 \mu \mathrm{J} \mathrm{cm}^{-2}\right.$ per pulse using $400 \mathrm{~nm}$ photons).

Figure 28 shows a comparison between the time-resolved photo-conductivity $\Delta \sigma(t)$ in regioregular-P3HT:PC 61 BM ( $r$ P $3 \mathrm{HT}: \mathrm{PC}_{71} \mathrm{BM}$ ), as determined by TRTS, and the time-resolved hole polaron density $n_{\mathrm{h}}(t)$, as determined by TA, using the known absorption cross-section of the hole 
polaron $^{85}$. As photo-induced carriers are generated in pairs, the hole polaron density, multiplied by a factor of 2, provides an estimate for the total number density $n(t)$ of the species giving rise to the TRTS photo-conductivity signal. Note that this implies that TRTS and TA monitor the same charged species, which may not be the case in general, and must be considered when analyzing the data. The combined TRTS and TA dataset may then be used to estimate the time-resolved TRTS carrier mobility $\mu(t)$, shown in Figure 29.

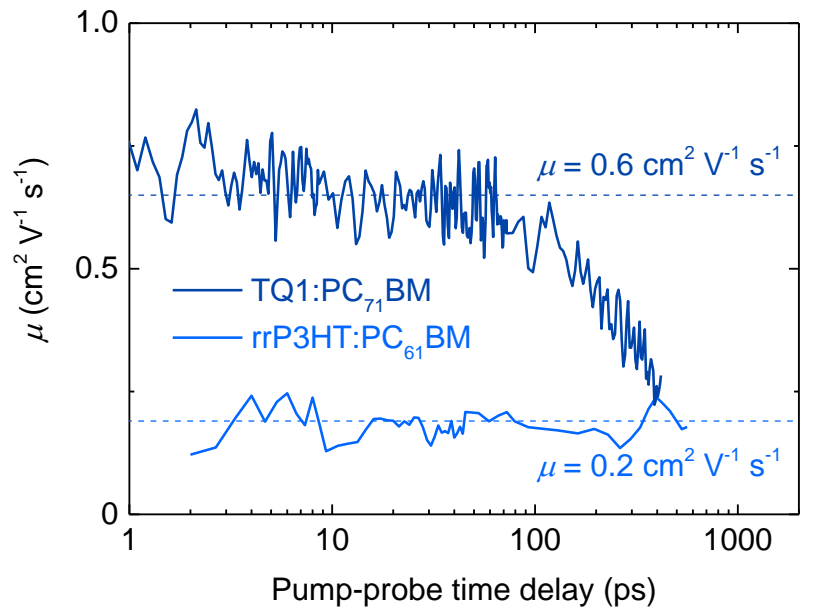

Figure 29. Transient mobility $\mu(t)$ estimated using TRTS and TA data for TQ1:PC ${ }_{71} \mathrm{BM}$ (darker blue) and rrP3HT:PC ${ }_{61} \mathrm{BM}$ (lighter blue). TQ1:PC 71 BM data are taken from ref. 17.

\section{TRTS Data Interpretation}

The mean TRTS mobility $\mu(t)$ is generally considered to be dominated by the significantly larger hole mobility in the polymer donor phase, and thus corresponds to the hole mobility. This assignment is based on the considerably lower electron mobility in neat $\mathrm{PC}_{61} \mathrm{BM}$ on a time scale of several picoseconds, as determined using Stark-shift spectroscopy $\left(\mu_{\mathrm{e}}=0.5 \times 10^{-2} \mathrm{~cm}^{2} \mathrm{~V}^{-1} \mathrm{~s}^{-1}\right.$ in the zero-field limit) ${ }^{16,51}$ and TRTS measurements on APFO3:PC 61 BM blends $\left(\mu_{\mathrm{e}}=0.3 \times 10^{-2} \mathrm{~cm}^{2} \mathrm{~V}^{-1} \mathrm{~s}^{-1}\right)^{52}$. Measurements on APFO3:PC ${ }_{61} \mathrm{BM}$ blends show increasing TRTS mobility with increasing polymer chain length ${ }^{52}$, whereas TRTS measurements on $\mathrm{P} 3 \mathrm{HT}: \mathrm{PC}_{61} \mathrm{BM}$ blends show only a minor effect of side chain regioregularity on TRTS mobility ${ }^{53}$. Both of these observations suggest that TRTS probes the hole mobility on the polymer backbone. As such, the $\operatorname{rrP} 3 \mathrm{HT}: \mathrm{PC}_{61} \mathrm{BM}$ trace in Figure 29, corresponds to the hole mobility. The remainder of this text solely describes what is observed for TQ1:PC ${ }_{71} B M$.

Although similar TRTS data, as for P3HT and APFO3, is not available for the main system studied in this thesis (TQ1:PC ${ }_{71} B M$ ), its TRTS mobility is also expected to be dominated by the hole mobility in the TQ1 polymer phase. This assignment is corroborated by TA measurements, where the 
redshift of the GSB, corresponding to hole thermalization, begins at a pump-probe delay of $\sim 100$ ps (see Figure 22a). The TRTS mobility in TQ1:PC ${ }_{71} B M$ also begins to decrease at 100 ps following photoexcitation. As such, the TRTS mobility data in Figure 29 is indicative of the onset of hole thermalization, whereas the subsequent mobility decay may be assigned to inter-site hole hopping. This assignment is merely a hypothesis and further studies are necessary to explain the decrease in the TRTS mobility in TQ1:PC ${ }_{71} \mathrm{BM}$.

There is also a complementary way to interpret the TRTS data, by considering the THz pulse as an electromagnetic wave. The photo-generated carriers are then under the influence of a time-varying $(\mathrm{AC})$ electric field of the $\mathrm{THz}$ pulse, which is attenuated by the photo-generated carriers. This is important to consider, since carrier mobility is frequency-dependent ${ }^{86}$. When probed by a high-frequency electric field, the charge carriers drift only a short distance during the period of the wave, predominantly hopping between sites of similar energy. In contrast, at low frequency the charge carriers are transported over a larger distance and are more prone to encounter high energy barriers (difficult hops), significantly reducing the charge carrier mobility. As such, the measured TRTS mobility describes charge motion over length scales of the order of $\sim 1 \mathrm{~nm}$ (ref. 87). Kinetic Monte Carlo modeling in paper III shows that the high probing frequency makes the TRTS mobility appear less time-dependent, as supported by earlier theoretical work on pristine materials ${ }^{88}$. The apparently time-independent TRTS mobility at the earliest time scales (1-100 ps), observed in typical OPV systems, may thus at least in part be due to the high frequency of the THz probe and not due to the complete absence of carrier thermalization. Further studies, disentangling the effect of the AC field on the observed kinetics, would elucidate the underlying physics.

To summarize, the combined TRTS and TA experiment provides an estimate for the mean of the hole and electron mobilities at $\mathrm{THz}$ frequencies on a 1-1000 ps time scale following photo-generation. There is growing experimental evidence in literature, suggesting that in polymer/fullerene BHJs the TRTS transients are mainly dominated by hole motion in the polymer phase. Due to the high frequency of the $\mathrm{THz}$ probe and the low carrier mobility in organic semiconductors, TRTS probes charge motion over relatively short length scales, i.e. of the order of $\sim 1 \mathrm{~nm}$, thus providing an estimate for the 'local' carrier mobility in an OPV film ${ }^{87}$. 


\subsection{Time-Resolved Microwave Conductance (TRMC)}

$20 \mathrm{~ns}-1 \mu \mathrm{s}$

Following the earliest time scales of charge separation and motion away from the donor-acceptor interface, as probed by the TRTS technique, subsequent charge transport and recombination can be monitored by the use of a microwave probe ${ }^{89}$. Time-Resolved Microwave Conductance (TRMC) measurements typically span the range of $20 \mathrm{~ns}-1 \mu \mathrm{s}$ following photo-generation. This temporal range is particularly suited for the investigation of charge separation and transport at later time scales and over larger distances, as compared to those probed by TRTS. In this thesis TRMC measurements were performed in order to evaluate the photo-generated carrier mobility in the temporal range of $20 \mathrm{~ns}-1 \mu \mathrm{s}$.

TRMC measurements were performed by the use of a microwave cavity and a continuous microwave source. The OPV film coated on a quartz substrate was placed inside the cavity at the position of the maximum absorption of microwave radiation. The interaction of the incident microwaves with the OPV film results in a decrease in the power of the incident microwave $P$, these changes are recorded and can be related to the photo-conductance $\Delta G(t)$ of the OPV film ${ }^{89}$

$$
\frac{\Delta P(t)}{P}=-K \Delta G(t)
$$

where $\Delta P(t) / P$ is the normalized change in microwave power reflected by the cavity and $K$ is the sensitivity factor of the cavity. The $K$ factor describes the optical cavity: it depends on the parameters of the cavity with the sample inside and is modelled by numerically solving the Maxwell equations. Following this procedure, the time-resolved photo-conductance of the OPV film $\Delta G(t)$ can be related to its time-resolved photo-conductivity ${ }^{89}$

$$
\Delta G(t)=\beta d \Delta \sigma(t)
$$

where $\beta$ is the ratio of the inner dimensions of the cavity in the sample plane ( $\beta=2.08$ for the cavity used in this thesis) and $d$ is the sample thickness.

Figure 30 shows a comparison between the time-resolved photo-conductivity $\Delta \sigma(t)$, as determined by TRMC, and the time-resolved hole polaron density $n_{\mathrm{h}}(t)$, as determined by TA. Paper I shows that the hole polaron decay $n_{\mathrm{h}}(t)$ is bimolecular (Figure 25). Therefore, the total number density $n(t)$ of the charged species giving rise to the TRMC photo-conductivity signal can be estimated as $n_{\mathrm{h}}(t) \times 2=n(t)$. Note that, as in the case of TRTS transients, this implies that TRMC and TA monitor the same charged species. The combined TRMC and TA datasets can then be used to estimate the average of the hole and electron mobilities, shown in Figure 31. 


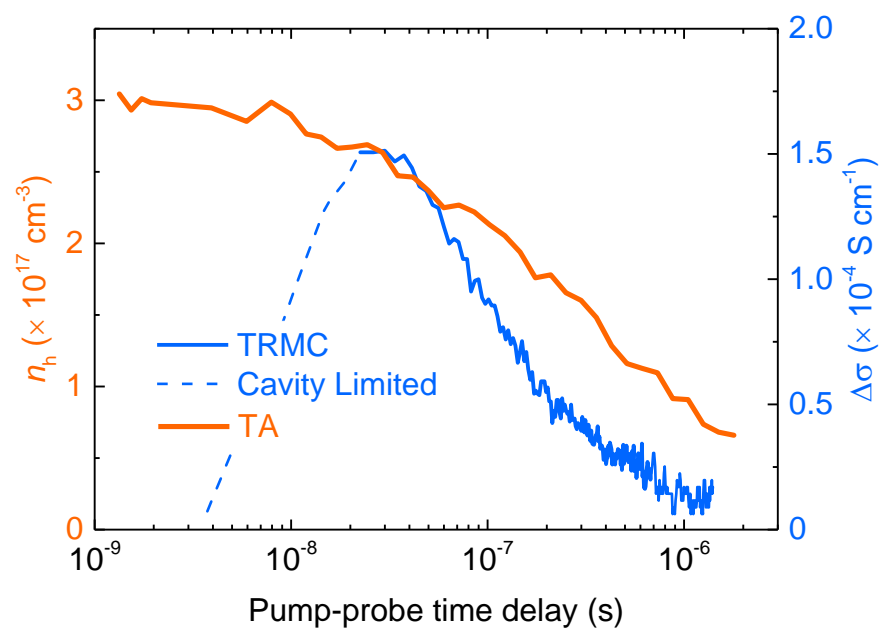

Figure 30. Comparison of the hole polaron density, as determined by TA (orange trace), and TRMC photo-conductivity (blue trace) transients recorded at a very similar excitation fluence $\left(5 \times 10^{12}\right.$ photons $\mathrm{cm}^{-2}$ per pulse).

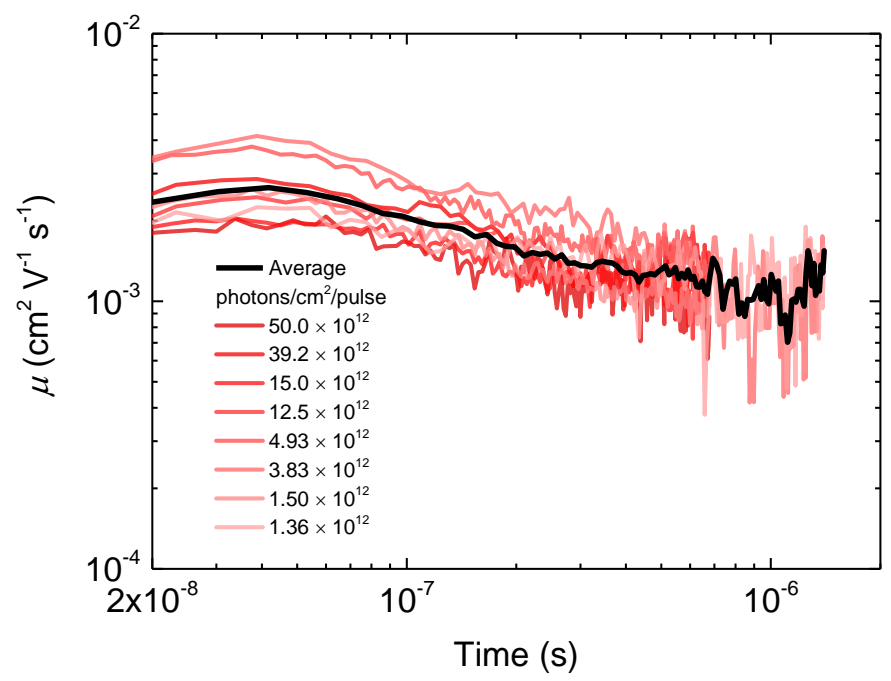

Figure 31. Estimated transient TRMC mobility is independent of pump-fluence within a factor of $\sim 2$. Adapted from ref. 57. 


\section{TRMC Data Interpretation}

Due to the considerably lower frequency of the electric field in a microwave probe $(\mathrm{GHz})$, compared to that in a Terahertz probe ( $\mathrm{THz}), \mathrm{AC}$ effects, due to the time-varying electric field of the microwave, are expected to be significantly less important in TRMC transients. Earlier work ${ }^{86,88}$ and simulations in paper III corroborate this observation. Therefore, the measured TRMC mobilities in OPV films can be more directly compared to those obtained by other DC (constant applied electric field) mobility techniques.

However, it has to be kept in mind that since TRMS probes charge motion only during a fairly limited temporal range, the photo-created charges will traverse only a limited distance during this time, i.e. the TRMC technique probes the carrier mobility over some 'local' spatial scale, similar to TRTS measurements. In that sense, the TRMC mobility may exceed that recorded by DC mobility techniques.

For the TQ1:PC 71 BM system studied in this thesis a time-dependent mobility on the $20 \mathrm{~ns}-1 \mu \mathrm{s}$ time scale is observed (Figure 31). At these time scales most of the thermalization has already taken place - the TRMC mobility decays only by a factor of $\sim 2.5$ over the measured range, as confirmed by Kinetic Manto Carlo simulations (Figures 21 and 22b). Paper II shows that the hole mobility in TQ1:PC $\mathrm{C}_{71} \mathrm{BM}$ is considerably lower than the electron mobility. This is generally the case when fullerene acceptors are employed. Therefore, the TRMC mobility is expected to be dominated by the considerably larger electron mobility.

In contrast, Savenije et al. have suggested that for regioregular-P3HT:PC ${ }_{61} \mathrm{BM}$ ( $\mathrm{rrP} 3 \mathrm{HT}: \mathrm{PC}_{61} \mathrm{BM}$ ) blends the TRMC mobility is time-independent at these time scales ${ }^{19}$. In general, the lower the energetic disorder of the material, the less important are non-equilibrium effects due to carrier thermalization $^{56}$. It may be expected that the TRMC mobility in $\operatorname{rrP3HT:PC}{ }_{61} B M$ is time-independent at least in part due to the lower energetic disorder of $\mathrm{rP}^{\mathrm{PHT}} \mathrm{PC} \mathrm{C}_{61} \mathrm{BM}$, compared to that in TQ1:PC ${ }_{71} \mathrm{BM}$. A comparison between the two systems was not done in this thesis but warrants further study.

TRMC also allows for additional means of detecting whether the photo-created charges have reached quasi-equilibrium at the $20 \mathrm{~ns}-1 \mu \mathrm{s}$ time scale. This can be accomplished by pump-fluence dependent TRMC mobility measurements. Carrier mobility in disordered organic semiconductors is known to be strongly concentration-dependent at quasi-equilibrium ${ }^{59,60}$, whereas this is not necessarily the case when out-of-equilibrium. Non-thermalized photo-generated carriers reside at higher energies in the DOS where considerably more sites are accessible for transport, significantly diminishing concentration effects. This is why mobility simulations in Figures 21 and $22 \mathrm{~b}$ are independent of the initial carrier density. TRMC measurements performed in paper III confirm that the photo-generated carrier mobility is concentration-independent (within experimental error) up to carrier densities of approximately $\sim 1 \times 10^{18} \mathrm{~cm}^{-3}$ (Figure 31). Therefore, at the $20 \mathrm{~ns}-1 \mu \mathrm{s}$ time scale the photo-generated carriers in $\mathrm{TQ1}: \mathrm{PC} \mathrm{C}_{71} \mathrm{BM}$ have not yet reached quasi-equilibrium. 
To summarize, the combined TRMC and TA measurement allows to estimate the mean carrier mobility on the $20 \mathrm{~ns}-1 \mu \mathrm{s}$ time scale following photoexcitation. In OPVs employing fullerene acceptors, TRMC mostly monitors electron transport, since the hole mobility is typically much lower. The presence or the lack of photo-generated carrier thermalization can be identified.

\subsection{Photo-CELIV}

$10 \mu \mathrm{s}-100 \mu \mathrm{s}$

In this thesis the carrier mobility and recombination dynamics at the latest time scales following photoexcitation (10 $\mu \mathrm{s}-100 \mu \mathrm{s}$ ) were monitored by the Photo-induced Charge Extraction by Linearly Increasing Voltage (Photo-CELIV) technique ${ }^{14,90}$. Photo-CELIV measurements allow to determine the time scale at which the photo-generated carriers have reached quasi-equilibrium. The Photo-CELIV technique is quite convenient as measurements are performed on complete OPV devices. The results can then be compared to other measurements performed on the same device, e.g. TA spectroscopy or TREFISH measurements. In this thesis the combined TREFISH and photocurrent measurements were performed on the same device as Photo-CELIV measurements.

In Photo-CELIV the photo-generated charges are created by a laser pump pulse and, after a variable time delay are extracted by a linearly increasing voltage pulse (Figure 32). It is important that the laser-induced photocurrent signal (sharp grey peak in Figure 32) would not overlap with the device response to the linearly increasing voltage, otherwise the signal is distorted. This limits the minimum achievable time delay, which in this thesis was $10 \mu \mathrm{s}$.
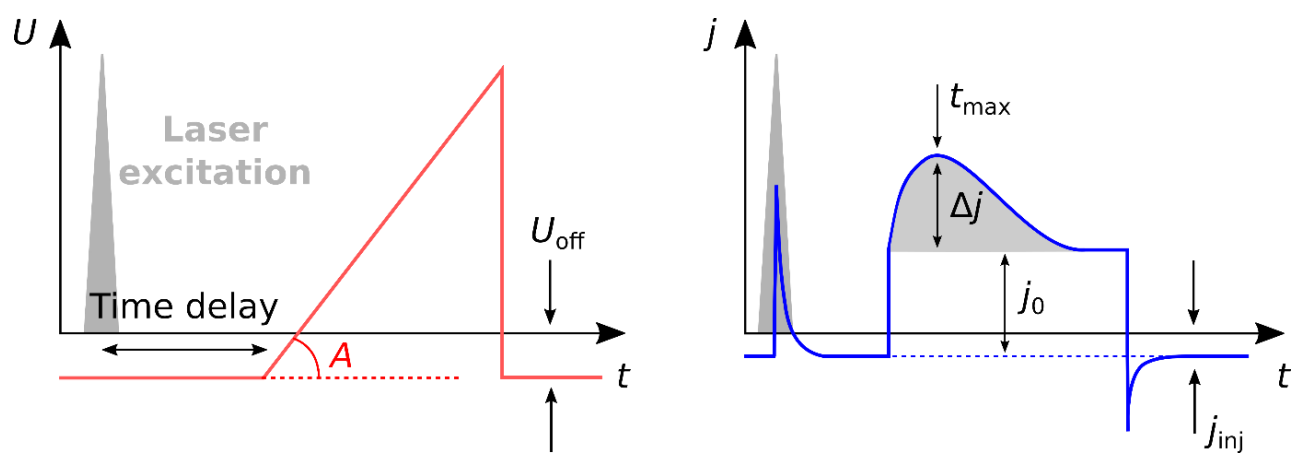

Figure 32. Photo-CELIV measurement schematic.

To avoid photo-generated charge leakage out of the device during the time delay a constant positive offset voltage $\left(U_{\text {off }}\right)$ is applied, typically similar to the open-circuit voltage $V_{\text {oc }}$ of the OPV device. However, the applied offset voltage may induce charge injection from the contacts $\left(j_{\text {inj }}\right)$. Therefore, $U_{\text {off }}$ must be chosen such that photo-generated charge leakage and injection currents are minimized. Generally speaking, this is quite challenging, but can be accomplished ${ }^{20,81,91}$. 
The mean mobility of the photo-generated charges can be estimated from the temporal position of the extraction current maximum $\left(t_{\max }\right)$. In this thesis the Photo-CELIV mobility was estimated as suggested by Juška et al. ${ }^{14}$

$$
\mu=\left(\frac{2}{3} \frac{d^{2}}{A t_{\max }^{2}}\right) /\left(1+0.36 \frac{\Delta j}{j_{0}}\right)
$$

where $A$ is the voltage ramp speed, $d$ the sample thickness, $\Delta j$ the maximum extraction current, $j_{0}$ the dark current, see Figure 32. As this technique is insensitive to whether the photo-generated holes or electrons are probed, the estimated mobility reflects the mean carrier mobility.
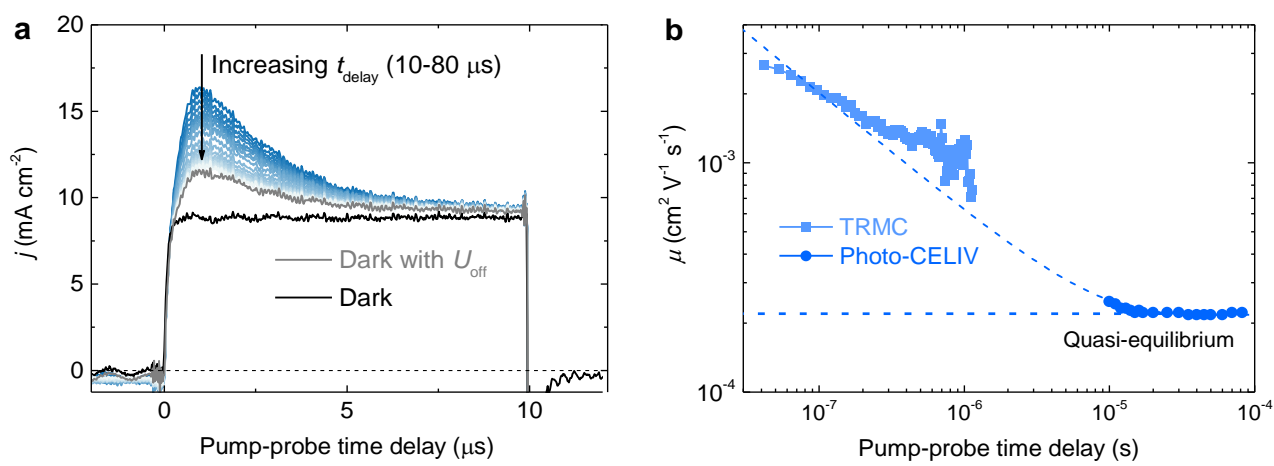

Figure 33. (a) Photo-CELIV transients of a TQ1:PC $\mathrm{P}_{71} \mathrm{BM}$ device. (b) Comparison of time-resolved TRMC and Photo-CELIV mobilities. Dashed lines are a guide to the eye. Panel $b$ adapted from ref. 57.

In this thesis the Photo-CELIV measurements were mainly used to determine the time scale at which the photo-generated charge carriers reach quasi-equilibrium. Figure 33a shows typical Photo-CELIV transients recorded on a TQ1:PC 71 BM device, whereas Figure 33b shows a comparison of the transient Photo-CELIV mobility to that determined by TRMC. The dashed lines are a guide to the eye, indicative of a transition from non-equilibrium charge motion driven by thermalization to quasi-equilibrium where thermalization is complete, resulting in a time-independent carrier mobility. Figure 33b shows that photo-generated carrier thermalization in OPV devices can take as long as $\sim 10 \mu$ s to complete.

Besides monitoring the mobility of the photo-generated charges, Photo-CELIV measurements can also be used to monitor the charge carrier recombination dynamics. By integrating the extraction current $(\Delta j)$ at a variable time delay, the charge carrier concentration decay with time $n(t)$ is obtained, see section 7.1 (Figure 25).

To summarize, Photo-CELIV measurements allow to determine both charge carrier mobility and recombination dynamics on microsecond time scales. At these time scales the photo-generated carriers are expected to be almost fully thermalized in their respective DOS. As such, Photo-CELIV transients can be used as a measure for the time scale at which thermalization completes. 


\section{Mobility Measurements without Photoexcitation}

Previously discussed experimental techniques monitor the transient mobility of photo-created charges following an excitation by a laser pulse. Alternatively, mobility measurements can also be performed under steady-state conditions in the dark, i.e. without photoexcitation. In this case the charge carriers are injected from the electrodes and, after traversing the OPV film, extracted at the second electrode. The most widely adopted mobility measurement technique used to describe operating OPVs (discussed below) relies on the assumption that the mobility of photo-created charges is the same, or least comparable to that of charges injected from the electrodes the dark. This chapter summarizes the main findings of paper IV, which shows that in most cases this notion is erroneous.

\subsection{Space-Charge Limited Currents (SCLC)}

Steady-state

Space-Charge-Limited Currents (SCLC) is arguably the most widely used technique to determine the steady-state charge carrier mobility in OPVs. The SCLC method relies on the use of electrodes that are Ohmic for only one type of carrier (hole- or electron-only SCLC). This facilities the injection of the selected carrier from the electrode into the OPV film. The injected charge carriers, following charge transport through the film, are extracted at the second electrode. By a suitable choice of electrodes the hole or the electron mobility can be determined. This is advantageous, as it is generally quite challenging to distinguish the electron and hole contributions in time-resolved experiments - SCLC provides useful complementary information.

The theory of space-charge-limited currents for an insulator sandwiched between parallel electrodes was first given by Mott and Gurney and is commonly referred to as the Mott-Gurney law $^{92}$. In its simplest and most widely used form the SCLC carrier mobility $\left(\mu_{\mathrm{scLC}}\right)$ can be estimated from

$$
j=\frac{9}{8} \varepsilon \varepsilon_{0} \mu_{S C L C} \frac{\left(U-U_{b i}\right)^{2}}{d^{3}}
$$

where $\varepsilon_{0}$ is the vacuum permittivity, $\varepsilon$ the dielectric constant of the active layer, $d$ the active layer thickness, $\left(U-U_{b i}\right)$ the applied voltage corrected by the built-in voltage of the device.

The presence of the SCLC regime can be determined by inspecting the slope of the current-voltage characteristic of the single-carrier diode in a log-log plot. Since $j \propto U^{2}$, a slope of 2 is indicative of the SCLC regime, in which case the above formula can be used to estimate the charge carrier mobility. For other cases, a more sophisticated formalism, taking account the presence of traps ${ }^{93}$ and/or an electric-field-dependent mobility ${ }^{94}$, must be employed. Figure 34 a (see the next page) shows the single-carrier device IV characteristics for the blends studied in paper IV. 
a
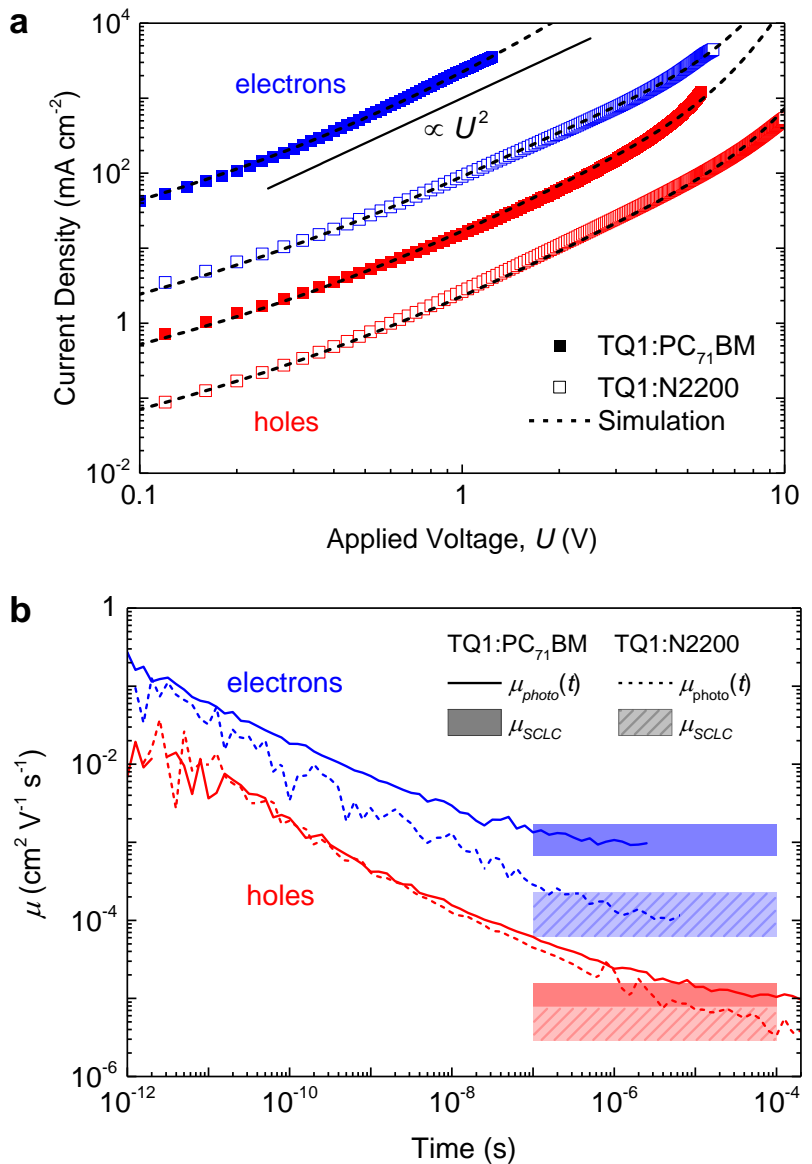

Figure 34. (a) IV characteristics of hole- and electron-only diodes based on TQ1:PC 71 BM and TQ1:N2200 measured in the dark. (b) Comparison of the time-dependent photo-generated carrier mobility to that of carriers injected from the electrodes in the dark, as determined by SCLC (shaded areas). Adapted with permission from ref. 56 .

In paper IV we have directly compared the mobility of photo-created charges, as determined by a combination of TREFISH and photocurrent measurements, to the mobility of charges injected from the electrodes in the dark, as determined by SCLC (Figure 34b). The photo-generated carrier mobility exceeds the SCLC mobility by several orders of magnitude, especially at early times following photoexcitation. Given the significant difference, it is questionable whether photo-generated charge transport can be described by the SCLC method.

The error in using the SCLC mobility to describe photo-generated charge transport can be quantified by comparing the carrier drift distance with and without thermalization 


$$
\begin{gathered}
d_{\text {photo }}(t)=\frac{U-U_{\mathrm{bi}}}{d} \int_{0}^{t} \mu_{\text {photo }}(t) d t \\
d_{S C L C}(t)=\frac{U-U_{\mathrm{bi}}}{d} \mu_{S C L C} t
\end{gathered}
$$

Equation 20 corresponds to the carrier drift distance when using the time-dependent mobility of photo-created charges $\mu_{\text {photo }}(t)$, whereas Equation 21 corresponds to the use of the SCLC mobility (or $\mu_{\text {photo }}(t)$ at long time delays).
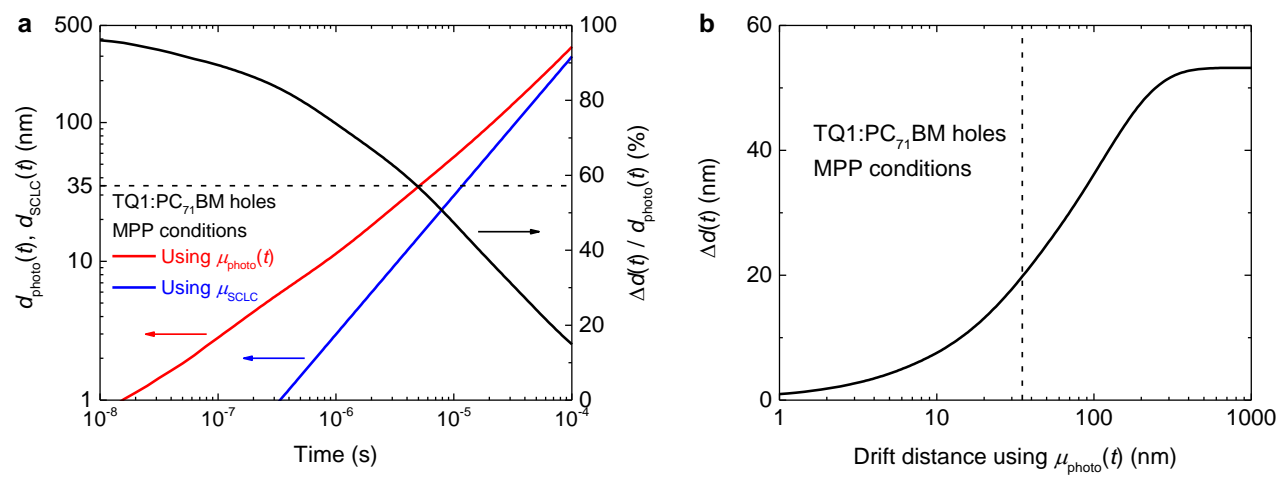

Figure 35. (a) Comparison of the estimated hole drift distance when either using the photo-generated $\mu_{\text {photo }}(t)$ or steady-state $\mu_{\text {SCLC }}$ mobility in a TQ1:PC ${ }_{71} \mathrm{BM}$ photovoltaic device at maximum-power point conditions. The black line shows the relative error. (b) The absolute error in using the steady-state SCLC mobility. Black dashed lines in both panels mark the drift distance to the extracting electrode $(=35 \mathrm{~nm})$ for an average photo-generated carrier in a $70 \mathrm{~nm}$ thick OPV device. Adapted with permission from ref. 56.

Figure $35 \mathrm{a}$ shows the resulting hole drift distance in $\mathrm{TQ1}: \mathrm{PC}_{71} \mathrm{BM}$ at maximum-power point conditions $(0.2 \mathrm{~V} / 70 \mathrm{~nm})$ using both methods. Since $\mu_{\text {photo }}(t)$ is significantly larger than $\mu_{\mathrm{sCLC}}$ at early times, photo-created charges drift a larger distance than quasi-equilibrated charges within the same period of time. Evidently, the use of $\mu_{\text {ScLC }}$ significantly underestimates the drift distance of the photo-generated carriers. The resulting error can be quantified as

$$
\begin{gathered}
\Delta d(t)=d_{\text {photo }}(t)-d_{S C L C}(t) \\
\frac{\Delta d(t)}{d_{\text {photo }}(t)}=\frac{d_{\text {photo }}(t)-d_{S C L C}(t)}{d_{\text {photo }}(t)}
\end{gathered}
$$

where $\Delta d(t)$ corresponds to the drift distance by which a quasi-equilibrated carrier is lagging behind a photo-created carrier, whereas Equation 23 quantifies the relative error. Figure 35b shows the resulting error versus the photo-generated carrier drift distance. The error is substantial. More concretely, the average photo-generated hole is extracted from a $70 \mathrm{~nm}$ thick 
OPV device at a drift distance of $d_{\text {photo }}(t)=35 \mathrm{~nm}$ (black dashed lines in Figure 35$)$, however, the use of a SCLC mobility would predict a drift distance of only $15 \mathrm{~nm}$ within the same period of time - a quasi-equilibrated hole is lagging behind the photo-generated hole by as much as $20 \mathrm{~nm}$ (Figure 35b), a significant number for a thin-film photovoltaic device.

In general, the smaller the energetic disorder of the material, the smaller the error in using a quasi-equilibrium carrier mobility will be (Figure 36). Since the GDM formalism employed here is applicable to any disordered organic semiconductor, kinetic Monte Carlo simulations allow us to predict the error for materials with different disorder values than those found in TQ1:PC ${ }_{71} B M$ and TQ1:N2200.

Figure 37 shows the predicted error for a hypothetical material with the indicated disorder at short-circuit $(1 \mathrm{~V} / 100 \mathrm{~nm})$ and at maximum-power point $(0.2 \mathrm{~V} / 100 \mathrm{~nm})$ conditions at a photo-generated carrier drift distance of $d_{\text {photo }}(t)=50 \mathrm{~nm}$. The error is larger at short-circuit conditions, as the gain in drift distance with increasing electric field strength is larger for the photo-generated charges (Equations 20 and 21). The error is decreasing with decreasing disorder due to the narrowing of the DOS distribution, making $\mu(t)$ become less time-dependent (inset Figure 37). For low disorder values the effect of thermalization on carrier transport becomes less important.

Although what error in $d_{\text {photo }}(t)$ is acceptable is not entirely clear, in paper IV we have, for illustrative purposes, chosen $15 \%$ of the average carrier drift distance in a typical $100 \mathrm{~nm}$ thick active layer, i.e. $15 \% \times 100 \mathrm{~nm} / 2=7.5 \mathrm{~nm}$ (green shaded area in Figure 37). This shows that at MPP conditions $(0.2 \mathrm{~V} / 100 \mathrm{~nm})$ the use of SCLC, or any other slow time-resolved or quasi-equilibrium mobility data, is acceptable only for materials with a sufficiently low energetic disorder $(\sigma<71 \mathrm{meV})$.

Such low energetic disorder values have been reported for only a few material combinations: PBDTTT-C:PC 71 BM $\left(\sigma_{\mathrm{h}}=70 \mathrm{meV}, \sigma_{\mathrm{e}}=75 \mathrm{meV}\right)^{95}$ and $\mathrm{rrP} 3 \mathrm{TH}: \mathrm{PC}{ }_{61} \mathrm{BM}\left(\sigma_{\mathrm{h}}=33 \mathrm{meV}, \sigma_{\mathrm{e}}=43 \mathrm{meV}\right)^{96}$. The blends studied in this thesis and previous work TQ1:PC ${ }_{71} B M$, TQ1:N2200, MDMO-PPV:PC 61 BM, and PCDTBT:PC 61 BM fall outside this range ${ }^{57,66,67}$. Furthermore, at higher electric field strengths, such as short-circuit conditions, even lower disorder values $(\sigma<56 \mathrm{meV})$ are required for a reliable description. Since most OPVs typically have an energetic disorder similar to or larger than $\sigma=56-71 \mathrm{meV}$, the use of SCLC mobility data to describe disordered OPVs is questionable even at relatively weak electric fields. Quasi-equilibrium mobility data are only reliable for materials with a sufficient low energetic disorder and are invalid for highly disordered materials. Figure 36 schematically summarizes the main findings of paper IV. 


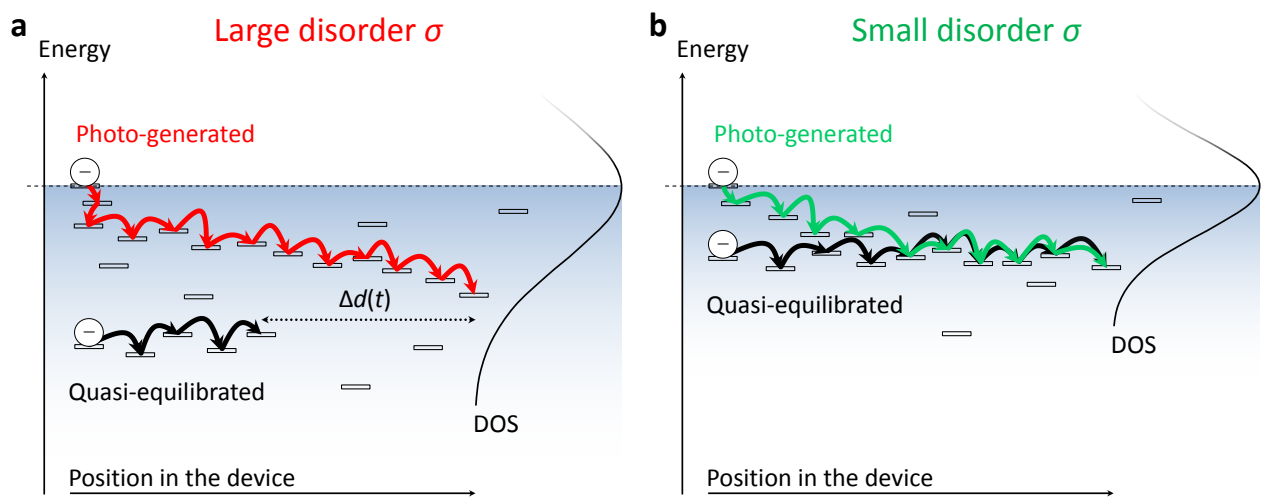

Figure 36 (a) In materials with a large energetic disorder, the photo-generated carriers drift a significantly larger distance than quasi-equilibrated carriers within the same period of time - the quasi-equilibrated carriers are lagging behind by $\Delta d(t)$. (b) In materials with a sufficiently low energetic disorder the transport of quasi-equilibrated carriers becomes comparable to that of the photo-generated carriers. Adapted with permission from ref. 56.

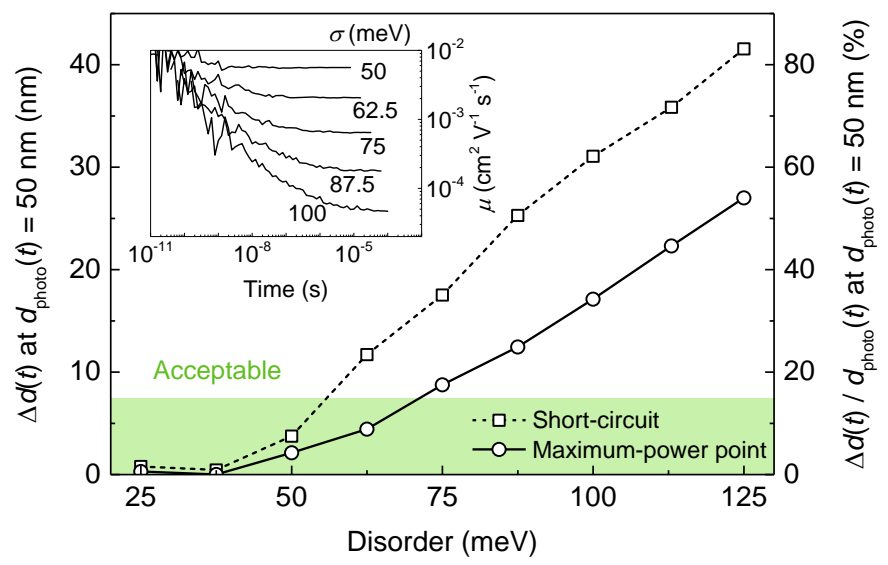

Figure 37. Predicted error for a hypothetical material with the indicated disorder. The error is calculated at short-circuit $(1 \mathrm{~V} / 100 \mathrm{~nm})$ and maximum-power point $(0.2 \mathrm{~V} / 100 \mathrm{~nm})$ conditions at a photo-generated carrier drift distance of $d_{\text {photo }}(t)=50 \mathrm{~nm}$. The inset shows that $\mu_{\text {photo }}(t)$ becomes less time-dependent with decreasing disorder, reducing the error. The green shaded area marks the suggested range of acceptable error. Adapted with permission from ref. 56. 



\section{Charge Transport in Pure and Mixed Phases}

Organic bulk heterojunction solar cells are known to have complicated multi-length scale morphologies ${ }^{97-100}$. However, the experimental data discussed so far (papers I to IV) were analyzed while assuming that the photoactive film can be treated as an effective medium, i.e. the nanoscale morphology of the BHJ film was not explicitly accounted for.

This approximation was justified for two reasons. First of all, relatively amorphous, i.e. rather well inter-mixed, donor/acceptor systems were chosen. Although such amorphous films may have nanoscale morphological variations, e.g. fullerene domains that are too small to resolve using microstructural characterization tools, the effective medium approximation remains valid. In any morphology, thermalization begins in the phase were the charge carrier is initially photo-created and transported, e.g., in an amorphous region, followed by charge transport and thermalization in an ordered region, since ordered regions are lower in energy. The extent of thermalization in each phase is set by its energetic disorder and the fraction of time the photo-generated carrier resides in each phase. Even in the case of complex morphologies, the use of an effective medium approximation can describe some average of the full thermalization kinetic, however, in this case morphology specific information is not explicitly captured by the model.

In order to understand how the morphology of the $\mathrm{BHJ}$ affects non-equilibrium charge motion, it would be desirable to investigate a set of samples where a rather well-defined $\mathrm{BHJ}$ morphology is varied in a controlled manner. This was, at least to some extent, accomplished in paper $\mathrm{V}$, where carefully co-sublimed BHJs, based on small-molecule donor/acceptor mixtures, were investigated. Although similar data, investigating the effect of donor/acceptor mixing on charge transport is available in literature ${ }^{5,97,98,101-103}$, previously reported studies are mainly semi-quantitative, in the sense that they do not answer the question of how pure do the neat domains have to be to facilitate efficient charge transport. The well-defined sample morphology in paper $V$ enabled quantitative analysis. Three important insights have been revealed, each is discussed in the sections that follow.

\subsection{Relation between Phase Purity and Charge Transport}

In paper $\mathrm{V}$ we have varied the molar fraction of $\alpha$-sexithiophene ( $\alpha-6 \mathrm{~T})$ in a buckminsterfullerene $\left(\mathrm{C}_{60}\right)$ matrix from homogeneously diluted ( $<10 \%$ molar), to a point where $\alpha$ - $6 \mathrm{~T}$ begins to form isolated aggregates $(>10-25 \%)$ or is strongly aggregated $(50 \%)$. We have thus varied the distance between isolated $\alpha-6 \mathrm{~T}$ sites and the level of disruption of the $\mathrm{C}_{60}$ phase in a controlled manner. The $\alpha-6 \mathrm{~T}: \mathrm{C}_{60}$ system can be viewed as a model for the mixed donor-acceptor phase in OPV. By a combination of TREFISH and photocurrent measurements, complemented with simulations based 
on the GDM model, we have quantified how donor/acceptor mixing affects the charge extraction kinetics (Figure 38).

The transient extraction data in Figure 38a indicate two extraction plateaus, most clearly visible for the $10 \%$ donor device. Given the approximate temporal position of these extraction plateaus (black dashed lines in Figure 38a), charge carrier mobilities of $\mu=0.5 \mathrm{~cm}^{2} \mathrm{~V}^{-1} \mathrm{~s}^{-1}$ and $\mu=6 \times 10^{-5} \mathrm{~cm}^{2} \mathrm{~V}^{-1} \mathrm{~s}^{-1}$, respectively, are expected. We have attributed the latter part of the transients to hole motion via isolated $\alpha-6 \mathrm{~T}$ sites (by a mechanism outlined in section 9.2), as confirmed by complementary hole-only mobility measurements using space-charge limited currents (Figure $38 \mathrm{c}$ inset), giving $\mu_{\mathrm{h}}=6 \times 10^{-5} \mathrm{~cm}^{2} \mathrm{~V}^{-1} \mathrm{~s}^{-1}$ at $10 \%$ molar donor. On basis of the high electron mobility reported in neat $C_{60}$ crystals $\left(\mu_{\mathrm{e}}=0.5 \mathrm{~cm}^{2} \mathrm{~V}^{-1} \mathrm{~s}^{-1}\right)$ (ref. 104), we have attributed the early part of the transients mainly due to the extraction of electrons. However, as will be explained in section 9.3 , a small fraction of holes ( $0-20 \%$, depending on bias) is also extracted very rapidly via $\mathrm{C}_{60}$.

The assignment of electron/hole extraction to the first/second plateaus in the transient extraction kinetics is similar to that used for polymer/fullerene and polymer/polymer blends in the previous chapters. This highlights that hole extraction in OPV devices, based on either polymer/fullerene, polymer/polymer or small molecule blends, is generally significantly slower than electron extraction.

The increasingly convex shape of the electron extraction plateaus with increasing $\alpha-6 \mathrm{~T}$ content indicates that the addition of $\alpha-6 \mathrm{~T}$ disrupts the $\mathrm{C}_{60}$ phase, increasing its energetic disorder (Figure $38 b)$. Figure 38c shows the corresponding effect on the photo-generated electron mobility (blue traces). With increasing $\alpha-6 \mathrm{~T}$ content electron transport undergoes a transition from non-dispersive (time-independent $\mu_{\mathrm{e}}$ ) to dispersive charge transport (time-dependent $\mu_{\mathrm{e}}$ ). This shows that, depending on the sample morphology, both charge transport regimes are possible in the same material.

More concretely, the addition of the donor below $10 \%$ molar does not hinder electron transport in the $C_{60}$ phase significantly - a quasi-equilibrium (long time) electron mobility of the order of $\mu_{\mathrm{e}}=1-2 \mathrm{~cm}^{2} \mathrm{~V}^{-1} \mathrm{~s}^{-1}$ is retained (Figure $38 \mathrm{c}$ ), similar to that reported in neat $\mathrm{C}_{60}$ crystals $^{104}$. In contrast, at donor fractions higher than $10 \%$, the quasi-equilibrium electron mobility is roughly an order of magnitude lower $\mu_{\mathrm{e}}=0.08 \mathrm{~cm}^{2} \mathrm{~V}^{-1} \mathrm{~s}^{-1}$ (at $25 \%$ molar) than at low donor content. Therefore, at a donor content below $10 \%$ molar the $C_{60}$ phase may be considered as effectively 'pure' for electron transport.

The electron disorder in the $\mathrm{C}_{60}$ phase at low donor content is considerably smaller than the disorder values determined for polymer/fullerene (TQ1:PC ${ }_{71} \mathrm{BM}$ ) and polymer/polymer (TQ1:N2200) systems discussed in the previous chapters. Due to the low disorder ( $\sigma \approx 60 \mathrm{meV}$ at $<10 \%$ donor) and on basis of Figure 38 , photo-generated electron transport in the $\mathrm{C}_{60}$ phase with low donor concentration can be reliably described by SCLC measurements in the dark. 
Surprisingly, increasing the donor content has a rather weak effect on hole extraction, with very similar hole mobilities in the range of 5.7-50\% donor (Figure 38c). Hole extraction is poor only at a donor content of $1.5 \%$. The physical mechanism, governing hole transport in these blends is discussed in the following sections.
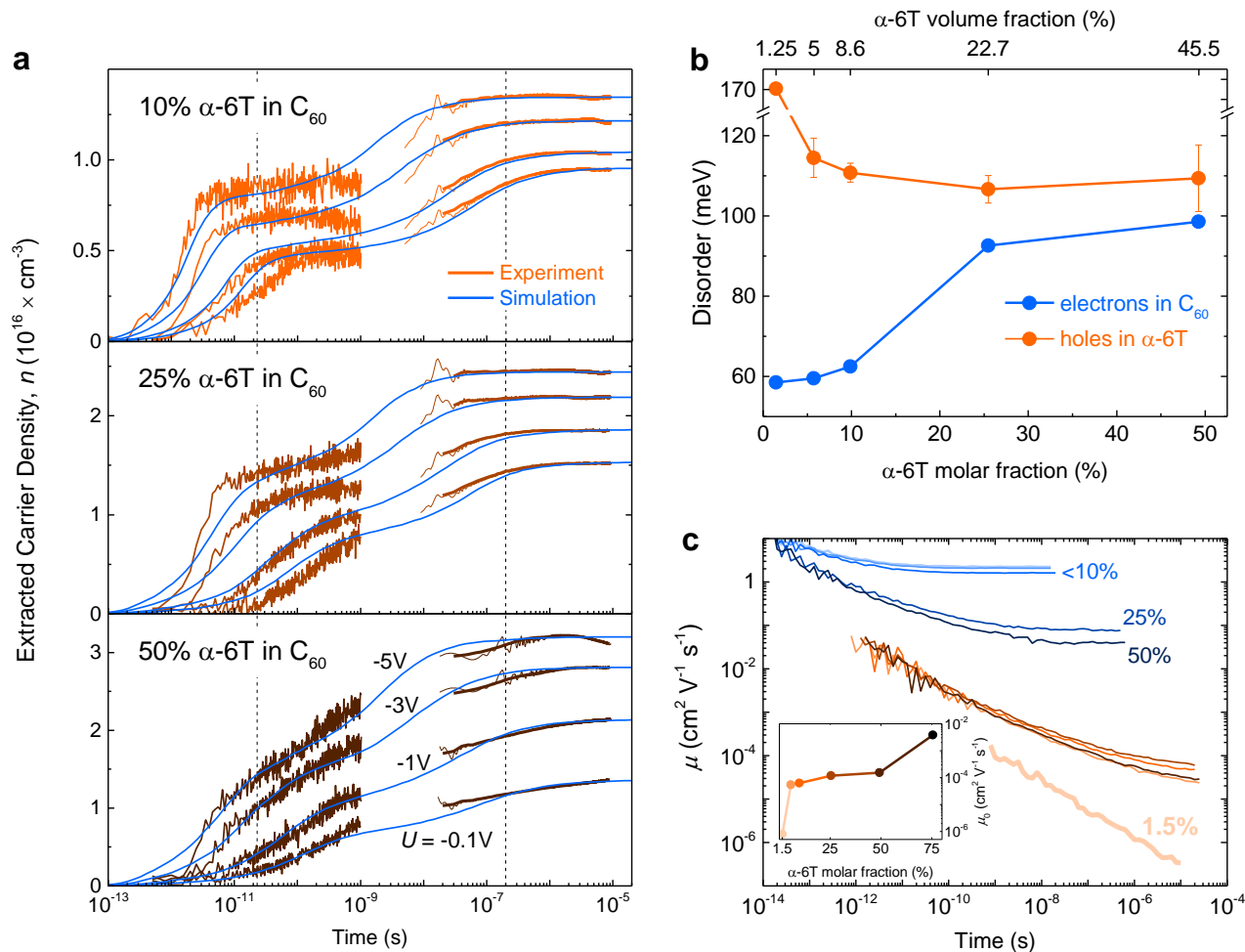

Figure 38. (a) Time-resolved charge extraction experiments at the indicated applied bias (orange traces), fitted by the Gaussian Disorder Model (blue traces) involving three charge populations: electrons in $\mathrm{C}_{60}$, holes in $\mathrm{C}_{60}$ and holes tunneling through $\mathrm{C}_{60}$ between isolated $\alpha$ - $6 \mathrm{~T}$ sites (see section 9.2). (b) Electron (blue) and hole (orange) energetic disorder versus $\alpha-6 \mathrm{~T}$ content. (c) Transient mobility of photo-generated electrons (blue) and holes (orange). The inset shows complementary SCLC measurements, showing the same trend as in the transient measurements. The increase in the SCLC mobility at $1.5 \%$ donor marks the onset for efficient long-range hole tunneling (see section 9.2). 


\subsection{Long-Range Hole Tunneling}

In paper $\mathrm{V}$ the transient measurement conditions were such that the hole was initially situated on an isolated $\alpha-6 \mathrm{~T}$ site. Generally speaking, given the strong localization in organic semiconductors, hole motion between isolated donor sites that are several nanometers apart $(\approx 3.4 \mathrm{~nm}$ at $5.7 \%$ molar dilution) should be quite unlikely. On the contrary, transient measurements reveal that when the mean center-to-center distance between isolated $\alpha$ - $6 \mathrm{~T}$ decreases from roughly $\approx 5.3 \mathrm{~nm}$ to $\approx 3.4 \mathrm{~nm}$ (corresponding to $1.5 \%$ and $5.7 \%$ molar dilution), efficient long-range hole tunneling through several $\mathrm{C}_{60}$ molecules occurs over distances as large as $\sim 4 \mathrm{~nm}$. This is clearly visible in the transient extraction kinetics, with a strong increase of the hole extraction peak at long time delays following photoexcitation (Figure 39).
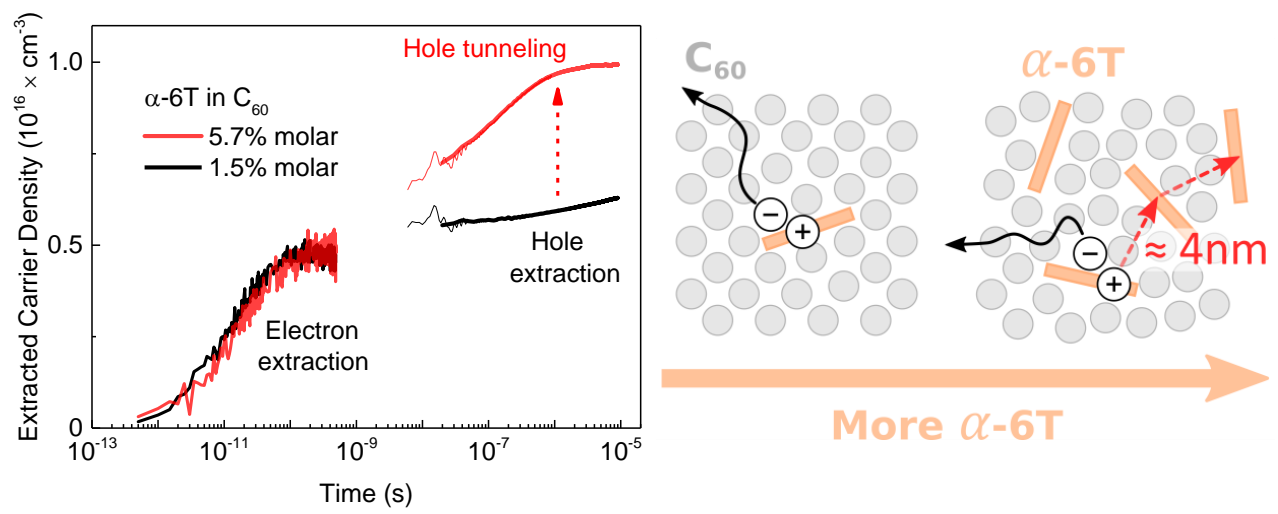

Figure 39. Combined TREFISH and photocurrent measurements reveal an onset for efficient hole tunneling (red dashed arrows in the schematic) at an inter-donor site distance of $\sim 4 \mathrm{~nm}$.

The combined transient and SCLC dataset in Figure 38c shows that hole transport between isolated donor sites occurs with a reasonably high hole mobility $\left(\mu_{\mathrm{h}}=5-15 \times 10^{-5} \mathrm{~cm}^{2} \mathrm{~V}^{-1} \mathrm{~s}^{-1}\right.$ for $5.7-50 \%$ donor). Since at low donor amounts ( $<10 \%$ molar) electron transport in the $\mathrm{C}_{60}$ phase remains unperturbed $\left(\mu_{\mathrm{e}}=2 \mathrm{~cm}^{2} \mathrm{~V}^{-1} \mathrm{~s}^{-1}\right)$, this data shows that $\mathrm{C}_{60}$ domains containing only a trace amount of material with a donating character enable ambipolar transport.

These results have several implications. First of all, the general notion that a continuous donor network is strictly necessary for hole transport in OPV devices is incorrect. Secondly, the commonly used terms of 'pristine', 'neat' or 'pure' phase require reevaluation. This is because, although $\mathrm{C}_{60}$ domains with $10 \%$ donor can be considered as effectively pure for electron transport, from the hole perspective, the same material, containing $90 \%$ of $\mathrm{C}_{60}$ would be typically regarded as 'not pure at all'. However, our data shows that even at such low amounts of isolated donor, the hole mobility is similarly high as at $50 \%$ molar donor $\left(\mu_{\mathrm{h}}=6-11 \times 10^{-5} \mathrm{~cm}^{2} \mathrm{~V}^{-1} \mathrm{~s}^{-1}\right.$ for $10-50 \%$ donor). Therefore, these results question the relevance of 'pristine' phases and whether a continuous interpenetrating donor/acceptor network is the ideal morphology for charge transport. 


\subsection{Hole Extraction via the Fullerene $\mathrm{C}_{60}$ Phase}

In paper $V$ we show that while the majority of the photo-generated holes in $\alpha-6 \mathrm{~T}: \mathrm{C}_{60}$ is transported between isolated donor sites by long-range hole tunneling through $\mathrm{C}_{60}$, at high reverse bias a small fraction $(0-20 \%)$ of the photo-generated holes is transferred to and extracted via the $\mathrm{C}_{60}$ phase.

The fraction of holes extracted via $\mathrm{C}_{60}$ can be estimated from the ratio in the extraction plateaus in the transient kinetics (Figure 40a). Since the photo-generated carriers are generated in pairs, a ratio of 0.5 means that the early time plateau is entirely dominated by the extraction of electrons. However, Figure 40a also shows that with increasing reverse bias the fraction of charges extracted at early times is increasing above 50\% (thick black horizontal line in Figure 40a). Given the high hole mobility reported in neat $\mathrm{C}_{60}$ crystals $\left(\mu_{\mathrm{h}}=2 \mathrm{~cm}^{2} \mathrm{~V}^{-1} \mathrm{~s}^{-1}\right)^{104}$, the bias-dependent increase of the early time extraction plateau was attributed to the rapid extraction of holes via $\mathrm{C}_{60}$.

a

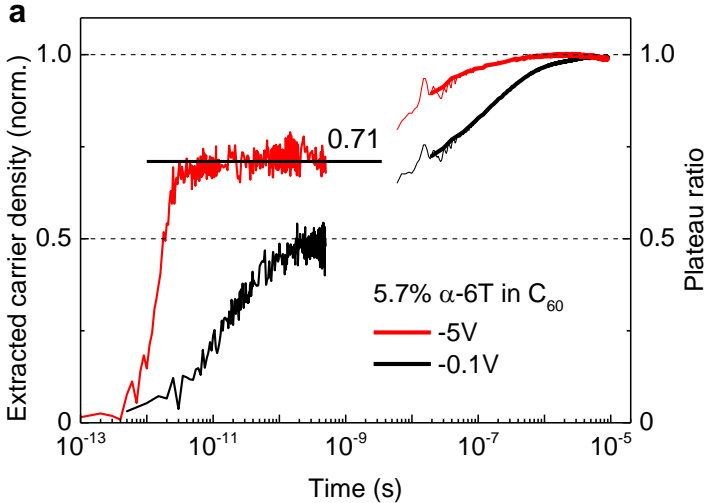

b Low $F$

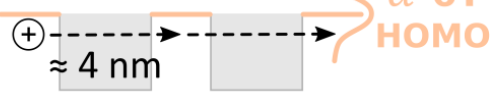

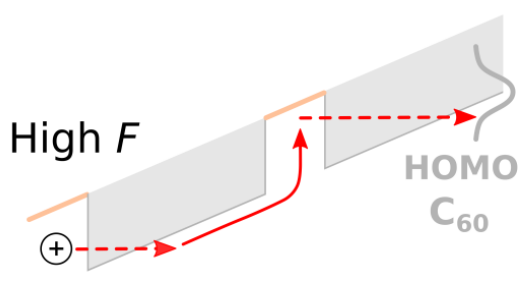

Figure 40. (a) Normalized transient extraction kinetics show the increasing contribution of the first extraction plateau with increasing reverse bias. (b) Schematic representation of the two mechanisms for hole transport in $\alpha-6 \mathrm{~T}: \mathrm{C}_{60}$. Black dashed arrows indicate long-range hole tunneling through $\mathrm{C}_{60}$ to nearby $\alpha-6 \mathrm{~T}$ sites, whereas red dashed arrows indicate hole de-trapping from $\alpha-6 \mathrm{~T}$ to $\mathrm{C}_{60}$ by Fowler-Nordheim-type tunneling, possible only at high electric fields $F$.

Based on the difference between the HOMO levels of $\alpha-6 \mathrm{~T}(-5.3 \mathrm{eV})$ and $\mathrm{C}_{60}(-6.2 \mathrm{eV})^{105}$, the energetic barrier for hole transfer from $\alpha-6 \mathrm{~T}$ to $\mathrm{C}_{60}$ is expected to be roughly $0.9 \mathrm{eV}=35 \mathrm{kT}$, meaning that, once the hole resides on $\alpha-6 \mathrm{~T}$, thermal de-trapping to $\mathrm{C}_{60}$ is incredibly unlikely. Instead, in paper $V$ we have suggested that hole de-trapping at high reverse bias occurs by Fowler-Nordheim-type tunneling ${ }^{106}$ through a triangular energy barrier (Figure 40b). Nevertheless, this process is only relevant at high reverse bias and not so important for operating OPV devices, see the next page. 


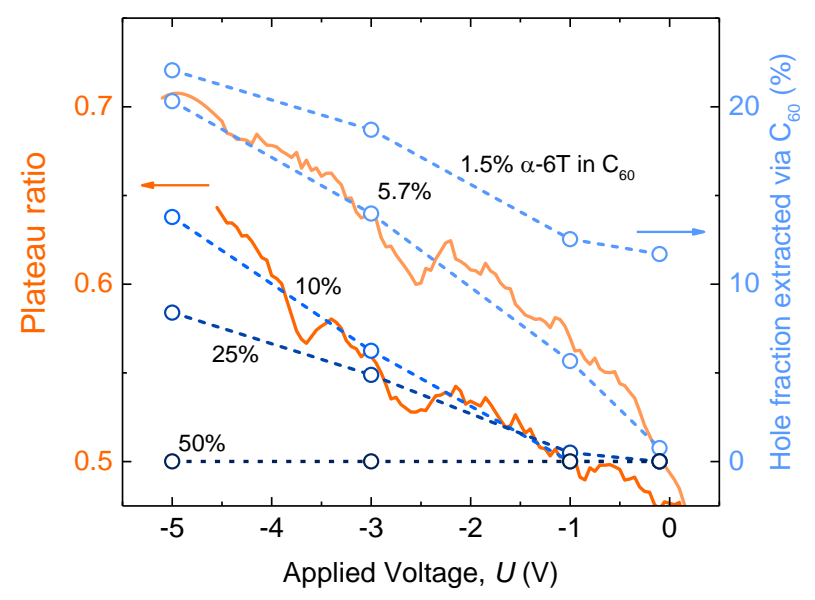

Figure 41. Extraction plateau ratio determined from experiments (orange, left $y$-axis) and the hole fraction extracted via $\mathrm{C}_{60}$ (blue, right $\mathrm{y}$-axis), as determined by GDM model fits to the transient kinetics in Figure 38.

A bias-dependent measurement in Figure 41 shows that, at field strengths relevant to OPV devices ( $U=0 \mathrm{~V}$ corresponds to short-circuit conditions), facile hole capture by isolated donor sites rapidly reduces the fraction of holes transported in $\mathrm{C}_{60}$ to zero. Upon trapping on isolated $\alpha-6 \mathrm{~T}$, low electric fields are insufficient to overcome the large energy barrier for hole de-trapping transfer to $C_{60}$, forcing all holes to move by long-range tunneling between isolated donor sites (section 9.2).

The possible importance of ambipolar transport in the fullerene phase of organic BHJ solar cells has been previously highlighted by several groups ${ }^{103,107,108}$, challenging the general notion that hole transport is strictly facilitated only by the donor, and electron transport only by the acceptor. Figure 41 shows that although $\mathrm{C}_{60}$ enables efficient hole transport at high reverse bias, the fraction of holes extracted via $C_{60}$ at relevant fields for OPV devices $(U=0 \mathrm{~V}$ corresponds to short-circuit conditions) is effectively zero. Therefore, at least for the case of small-molecule-donor and neat fullerene $\left(C_{60}\right.$ or $C_{70}$ ) OPV devices, hole extraction only via the fullerene phase can be ignored. However, long-range hole tunneling via isolated sites of a material with a donating character, e.g. donor molecules or impurities, must be considered. 


\section{Limitations of the Present Treatment}

Most of the experimental data presented in this thesis were fitted by the GDM model using kinetic Monte Carlo simulations. Nevertheless, the model has certain limitations, limiting the amount of insight that can be extracted from the experimental data. This chapter is intended to highlight some of those limitations and unpublished attempts to address those limitations.

\subsection{Bulk Heterojunction Morphology}

The simulations performed in this thesis assume that the $\mathrm{BHJ}$ film can be treated as an effective medium, i.e. the BHJ morphology is not explicitly accounted for. The effective medium approximation has turned out to be successful in describing most of the experiments presented in this thesis. However, we have found that, in particular for measurements monitoring the carrier recombination dynamics at conditions where the electric field is quite weak, e.g. in thin films or at 'flat-band' conditions in OPV devices, the effective medium approximation fails to describe the experiments.

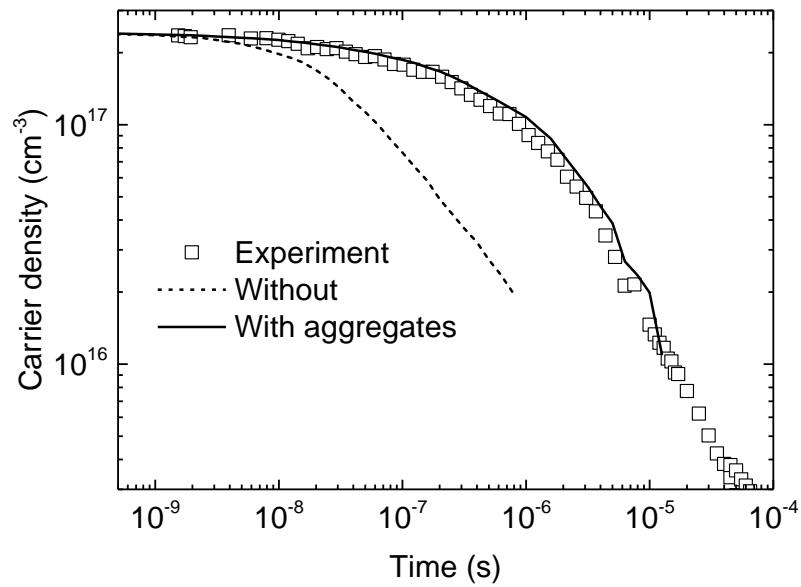

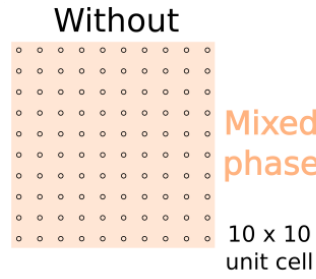

With aggregates

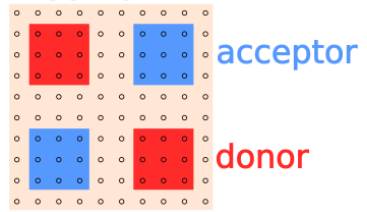

Figure 42. Experimental carrier recombination dynamics (square symbols) in a TQ1:PC ${ }_{71} B M$ device at 'flat-band' conditions can be explained only by a model incorporating small aggregated inclusions of donor and acceptor materials in a mixed donor/acceptor matrix (see schematic). Experimental data are shown at the lowest measured pump-fluence $\left(2.5 \times 10^{12}\right.$ photons $\mathrm{cm}^{-2}$ per pulse) in Figure 25 .

Figure 42 shows the previously described carrier recombination kinetics as measured by TA and Photo-CELIV in a TQ1:PC ${ }_{71} \mathrm{BM}$ device at 'flat-band' conditions, i.e. at a low electric field (Figure 25). The black dashed trace corresponds to a model prediction using the experimentally determined 
simulation parameters that have successfully described the charge extraction and thermalization data in TQ1:PC ${ }_{71} B M$ discussed in the previous chapters. However, here, the model fails to describe the experiment.

It is well documented in literature that aggregated donor and acceptor domains may suppress carrier recombination ${ }^{44,109-111}$. Therefore, we have modified our model to incorporate inclusions of aggregated donor and acceptor materials in an otherwise mixed $\mathrm{BHJ}$ matrix (Figure 42).

A total of four $3 \times 3$ inclusions, two for the donor, two for the acceptor in a $10 \times 10$ unit lattice describing the mixed donor/acceptor phase, were included. Figure 42 shows a top view of the lattice, i.e. these inclusions are pillars extending throughout the entire thickness of the BHJ film. The energy levels of the aggregated regions were set such that the photo-generated carriers are driven from the mixed phase into the aggregated regions, which were described by lower energetic disorder values than in the mixed phase. The reasonably good fit to experimental data shows that to simulate the carrier recombination dynamics at low electric fields, the $\mathrm{BHJ}$ morphology has to be taken into account, at least crudely, as shown here.

Note that the inclusion of the BHJ morphology was not so important when modelling the TREFISH and photocurrent measurements, as they are typically performed at short-circuit conditions ( 1V per $100 \mathrm{~nm}$ ) or high reverse bias, where charge carrier recombination is not severe (IQE in TQ1:PC ${ }_{71} \mathrm{BM}$ is $\approx 83 \%$ at short-circuit). In contrast, the BHJ morphology is important to consider at low electric fields, i.e. lower than at short-circuit conditions, where an increasing fraction of the photo-generated carriers undergo recombination. Taking into account the BHJ morphology in the model will be particularly important when describing the open-circuit voltage ( $\left.V_{o c}\right)$ of the operating device, since at open-circuit conditions all of the photo-generated carriers recombine.

\subsection{Curve under Continuous Illumination}

The most important question to answer is how the gradual thermalization of photo-generated carriers affects the IV curve and the open-circuit voltage ( $V_{0 c}$ ) of the OPV device under continuous illumination. In principle, this can be answered by simulating the IV curve by kinetic Monte Carlo, using simulation parameters obtained from fitting the transient data described in the previous chapters. This requires an appropriate description of both the photoactive layer morphology (see the previous section) and the properties of the extracting electrodes.

When simulating the transient extraction measurements, we have previously assumed that the electrodes act as sinks for the photo-generated carriers. In other words, only charge extraction was considered, whereas charge injection from the electrodes into the OPV film was not accounted for. This is justified on basis that charge extraction measurements were performed under short-circuit conditions or high reverse bias. However, in order to simulate the IV curve of an operating OPV device, the electrodes must allow for charge injection into the OPV film. Generally speaking, such IV simulations using kinetic Monte Carlo are very time consuming. This 
makes it particularly challenging to verify whether the properties at the contacts are described correctly.

Figure 43 shows some preliminary IV simulations, using kinetic Monte Carlo, for the TQ1:PC ${ }_{71} B M$ photovoltaic device under continuous illumination. Most of the simulation parameters, except those describing the contacts, were set to values obtained from fitting the transient data described in the previous chapters. Evidently, by taking into account the $\mathrm{BHJ}$ morphology, the FF of the simulated IV curve improves, however, it remains too low to describe the experimental IV curve.

Note that the agreement between the experimental and the simulated $V_{O C}$ is merely due to the fact that in the simulations the energetic position of the donor HOMO and acceptor LUMO levels, i.e. the effective bandgap of the organic solar cell, can be adjusted to fit the experiment. The mismatch between the experimental and the simulated $F F$ is considerably more important and must be accounted for.

We have, at the time of writing, tested various conditions to correct for the mismatch between the experiment and simulations, such as varying the carrier hopping parameters, reducing the carrier recombination rate, increasing the dielectric constant, adjusting the injection barrier heights and/or using the Marcus carrier hopping rate ${ }^{112}$. In all cases, the FF of the simulated IV curve does not exceed the one shown in Figure 43. We are now convinced that the properties at the injecting contacts are responsible for the mismatch in our current simulations. Further studies are necessary to relate the non-equilibrium phenomena described in this thesis to the properties of the IV curve.

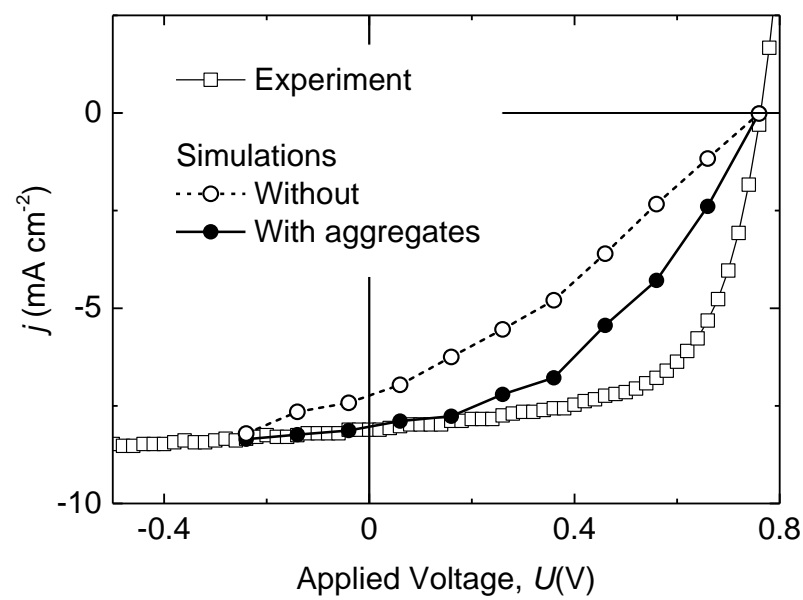

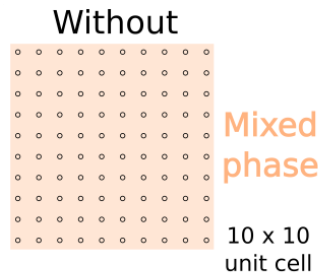

With aggregates

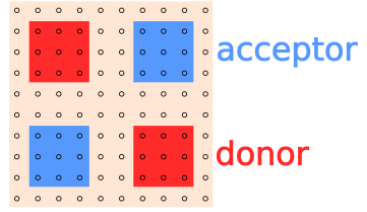

Figure 43. The IV curve of the TQ1:PC $C_{71} B M$ photovoltaic device under continuous $A M 1.5 \mathrm{~g}$ illumination in experiment (open squares) and simulated by kinetic Monte Carlo (circles). Simulations were performed when considering only the mixed donor/acceptor phase without aggregates (empty round symbols), and with small aggregated inclusions of donor and acceptor materials in an otherwise mixed donor/acceptor matrix (filled round symbols), shown in the schematic. 



\section{Conclusion}

The combined dataset presented in this thesis shows that charge transport in disordered organic solar cells is governed by the gradual thermalization of the photo-generated carriers. Charge extraction from an OPV device can be successfully described by a time-dependent carrier mobility. This was shown to be the case for polymer/fullerene, polymer/polymer and small-molecule-based $\mathrm{BHJ}$ organic solar cells.

In contrast, the use of quasi-equilibrium mobility values, such as those obtained by measurements without photoexcitation, is highly questionable in view of the typically large energetic disorder in OPV materials. Due to the dispersive nature of charge transport, even a properly defined mean carrier mobility is only an approximation of the underlying physics.

Although, according to our estimates, the photo-created carriers can be extracted from the OPV device before reaching quasi-equilibrium, this is of fairly limited use, as most of the excess photon energy is dissipated on ultrafast time scales by fast diffusive motion. Thermalization in the disorder broadened DOS should be avoided altogether. The solution, although it is not entirely evident how to accomplish this, is to reduce the energetic disorder. This would increase the maximum attainable electrical energy per electron-hole pair, and also increase both the transient and the quasi-equilibrium charge carrier mobility, leading to improved photovoltaic performance. Hopefully, the framework laid out in this thesis serves as motivation to continue this pursuit.

\section{Outlook}

Most of the experimental data presented in this thesis were collected on just a few material systems. The relevance of the non-equilibrium processes described here to other OPV systems was assigned largely on basis of related observations in literature and the generality of the GDM framework. Further experimental studies, using different materials, may not only reveal exceptions to this rule, but also provide further material design motifs for reduced energetic disorder ${ }^{113,114}$.

The results presented here also raise a number of questions that may serve as motivation for further studies. Each idea is discussed separately in the sections that follow. 


\section{Hot Carrier Organic Solar Cell}

One might think, given the results presented here, that the operation of the organic solar cell resembles that of a hot carrier solar cell, where the photo-created charges are extracted before their full thermalization takes place. This is correct, however, note that without appropriate electrodes, it is impossible to convert the extra energy stored in the non-thermalized carrier populations to electrical energy, i.e. it is impossible to gain in Voc.

This is largely due to the fact that regular Ohmic electrodes are 'cold' for the hot photo-generated carriers. Regular Ohmic electrodes allow for the exchange of photo-generated carriers between the absorber and the electrodes over energies in the absorber. The thermalization of hot photo-generated carriers in the electrodes then results in a significant loss of the photo-generated carrier energy to heat. In addition, the unimpeded exchange of cold electrons from the electrodes with the photo-generated carriers in the absorber further cools down the photo-generated carrier population.

To make use of the energy stored in non-thermalized carrier populations, requires energy-selective contacts, where the photo-generated carriers are extracted through a narrow energy range $\mathrm{e}^{115}$. Resonant tunneling through quantum dots or heterostructures is generally considered as the most viable solution in inorganic photovoltaics ${ }^{23}$. However, the actual implementation, i.e. a demonstration of a hot carrier solar cell working at practically relevant power conversion efficiencies, remains very challenging.

Note that, since most of the excess photon energy $(1-2 \sigma)$ in an organic solar cell is lost by ultrafast diffusive charge motion, to prevent this loss, the photoactive layer in an organic hot carrier cell would have to be extremely thin (roughly $<10 \mathrm{~nm}$ ) and operate mainly based on diffusion. The limited absorption of such a thin photoactive layer would pose constrains on the maximum attainable electrical power. The maximum attainable $\mathrm{V}_{\mathrm{OC}}$ of a hot carrier $\mathrm{BHJ}$ organic solar cell would be set by the charge-transfer state manifold. Organic solar cells where efficient charge separation does not take place via the $\mathrm{CT}$ state, e.g. those based on a single material, would loosen the $V_{\text {oc }}$ constraint.

\section{Relating the CT Manifold to the Density of States}

The energy of the CT state is known to set the $\mathrm{V}_{\mathrm{Oc}}$ of the OPV device ${ }^{31,32}$. In principle, the gradual thermalization of the photo-generated carriers, taking place in the DOS, also limits the $V_{0 C}$ (ref. 29). The two pictures should be somehow mutually consistent. Thus, it would be particularly helpful to elucidate how the energy of the CT manifold is related to the energies of the hopping sites in the carrier DOS.

Generally speaking, the DOS describes all of the hopping sites, whereas the CT manifold only corresponds to those sites that form a donor/acceptor interface. As such, the CT manifold 
corresponds to only a subset of sites in the collective DOS, i.e. the combined DOS of the donor HOMO and the acceptor LUMO. Although the properties of the donor/acceptor interface can be probed by spectroscopic tools, the rest of the DOS is typically invisible in such measurements and must be probed by alternative means. Describing the CT manifold and the DOS by a consistent formalism would elucidate the physics of organic solar cells further. The added benefit of such a study would be that it may also answer the question as to why, despite the gradual thermalization of photo-created charges, do the assumptions of detailed balance and quasi-equilibrium apply in describing the $\mathrm{V}_{\mathrm{OC}}{ }^{22,31}$.

\section{Temperature-dependent Charge Extraction Measurements}

Since photo-generated carrier thermalization takes place downward in energy, the initial thermalization dynamics should be insensitive to temperature. In contrast, the latest time scales of thermalization, where an increasing number of thermally activated (upward) hops occurs, the thermalization dynamics would be temperature-dependent. This hypothesis has already been confirmed by TREFISH and photocurrent measurements on neat polymer films ${ }^{74}$, nevertheless, it would be interesting to also perform such measurements on OPV devices.

\section{Charge Extraction Measurements using Different Photoexcitation Conditions}

The relevance of the combined TREFISH and photocurrent extraction data to OPV devices operating under continuous illumination conditions was assumed on basis of kinetic Monte Carlo simulations and the fact that the transient experiments were performed in the linear pump-fluence regime. Instead, a more direct measurement would be more convincing. For example, the combined TREFISH and photocurrent experiment could be performed on a photovoltaic device that is, in addition to the pump and the probe pulses, continuously illuminated, e.g. by a light-emitting diode (LED), corresponding to 1 sun illumination conditions.

Furthermore, it would be interesting to perform the combined TREFISH and photocurrent measurement under different pump wavelengths. Regardless of the photon energy, following exciton dissociation the electron is most likely to be transferred to the DOS center of the acceptor LUMO (or the donor HOMO for photo-induced hole transfer). Hence, the electron thermalization dynamics that follow photo-induced charge transfer, are expected to be insensitive to photon energy. The same argument holds for hole transfer. This is in a way already visible in the data presented in this thesis, where the transient measurements were performed at different excitation wavelengths. However, the measurements were performed on different OPV systems. An additional study, using only a single material system would verify the above hypothesis. 


\section{Improving Long-Range Hole Tunneling}

The limits of long-range hole tunneling remain to be explored. This development is particularly exciting as long-range hole tunneling loosens the requirements on the $\mathrm{BHJ}$ morphology.

In principle, the tunneling probability between isolated sites depends on the difference in the HOMO levels between the donor molecule and the host matrix ( $\mathrm{C}_{60}$ in paper $\mathrm{V}$ ). Therefore, hole transport via isolated donor sites could be enhanced by a suitable choice donor and host materials. It is worthwhile to investigate whether such long-range tunneling phenomena also occur in other OPV systems, including those not employing fullerene acceptors, and also whether long-range electron tunneling takes place between isolated acceptor sites. 


\section{Acknowledgements}

This thesis was conducted in 2012-2017 at the group of Biomolecular and Organic Electronics (BiOrgEl) led by Olle Inganäs, my thesis supervisor, to whom I would like to express my deepest gratitude. Olle, I thank you for the opportunity to join the group as a graduate student, I am proud to have been your student. Your critical thinking, innovative ideas and positive attitude are inspiring and have in many ways shaped my views on science and life. The continuous support and the personal freedom that you have given me have been invaluable to my personal and professional development.

This thesis would not have been possible without the help and support from my co-supervisor Martijn Kemerink, whom I consider to be an exemplary scientist. Martijn, I have thoroughly enjoyed working with you and have learnt a great deal from our discussions. Your creativity and sharp eye for detail have helped my projects run well. Your guidance has laid the foundation for the scientist that I am today.

I would like to thank the BiOrgEl crowd for the enjoyable time and all the help that I have received. In particular I would like to express my gratitude to: Wolfgang Tress, for my never ending questions regarding solar cell operation and your patience in diligently answering those questions (and other questions about life, particularly in times of despair). Zheng Tang, for your help and mentorship when I took my first steps in the lab. It seems that I have learned all there is about sample preparation from you and it has served me well throughout the rest of the thesis. L. Mattias Andersson, I cannot thank you enough for all the help regarding the time-resolved measurements, especially those that involve fixing broken lasers in Lund. Jonas Bergqvist, for asking those seemingly simple questions that turn out to be not that simple at all (including those on a 24-hour train ride to Austria). Fátima Ajjan, for your positive attitude, encouragement and in helping me through all the paperwork to finalize this thesis. Niclas Solin, for answering my chemistry-related questions (and the games of chess, I think I get to keep the positive score).

I thank the rest of the BiOrgEl crowd for the positive working environment and their invaluable help in the lab, especially during those late evenings when some crucial lab item seems to be missing. In no particular order and mixed with the CoMaDe group, I would like to thank: Hassan Abdalla, Nikolaos Felekidis, Olof Andersson, Guangzheng Zuo, Zaifei Ma, Liangqi Ouyang, Yuxin Xia, Thomas Österberg, Chiara Musumeci, Erica Zeglio, Anders Elfwing, Wanzhu Cai, Luis Ever Aguirre, Bian Qingzhen, Deping Qian, Feng Gao, Fengling Zhang.

The TREFISH measurements performed in Vidmantas Gulbinas lab in Vilnius were instrumental for the completion of this thesis. I would like to thank Vytenis Pranculis for his patience to my nagging requests to perform the measurements in a certain way. Andrius Devižis for mentoring me on how to perform the TREFISH measurements and Vidmantas Gulbinas for the fruitful collaboration and insightful discussions. 
I would like to thank our collaborators at Lund University for access to their ultrafast laser lab, the TA and TRTS measurements. In particular, I thank Carlito S. Ponseca Jr., Arkady Yartsev and Villy Sundström. Carlito, your cheerful attitude is contagious, thank you for proof-reading my thesis.

I also thank D.H.K Murthy and Tom J. Savenije at Delft University for the TRMC measurements and numerous discussions regarding those measurements. I thank my previous mentors Kęstutis Arlauskas and Gytis Juška for guiding me through the first steps in science as I was practicing the Photo-CELIV measurements. I am grateful to Frédéric Laquai, Fabian Etzold and Dominik Gehrig for the TA measurements and the lengthy discussions that follow those measurements. I also thank the Chalmers University crowd: Amaia Diaz de Zerio Mendaza, Christian Müller, Renee Kroon, Mats R. Andersson, Ergang Wang for the materials and the interesting projects. I thank Aaron M. Lindenberg and Alberto Salleo for hosting me at Stanford University to practice the TRTS measurements. Meng-Ju Sher, I thank you for your patience in mentoring me in the TRTS lab. Last but not least, I would like to thank our collaborators in Dresden: Koen Vandewal, Donato Spoltore and Johannes Benduhn for the fruitful collaboration on small molecule blends. I thank all of you, without your help none of this would have been possible.

I thank my office mates Mohammad J. Jafari and Andreas Skallberg for keeping our office a pleasant working environment. Andreas, I thank you for opening and closing the window whenever it is necessary (pun intended).

The administrative support of Mikael Amlé and Anna-Maria Uhlin was excellent, allowing me to mostly focus on science instead of administrative issues, thank you. I also thank my PhD thesis mentor Hans Högberg for his support and encouraging words.

Finally, a big shout-out goes to my friends, family and the Lithuanian crowd at IFM! 


\section{References}

1. Renewables 2016 Global Status Report. REN21 Available at: http://www.ren21.net/status-ofrenewables/global-status-report/. (Accessed: 1st April 2017)

2. Green, M. A. Commercial progress and challenges for photovoltaics. Nat. Energy 1, 15015 (2016).

3. Polman, A., Knight, M., Garnett, E. C., Ehrler, B. \& Sinke, W. C. Photovoltaic materials: Present efficiencies and future challenges. Science 352, aad4424 (2016).

4. Shockley, W. \& Queisser, H. J. Detailed Balance Limit of Efficiency of p-n Junction Solar Cells. J. Appl. Phys. 32, 510-519 (1961).

5. Liu, Y. et al. Aggregation and morphology control enables multiple cases of high-efficiency polymer solar cells. Nat. Commun. 5, 5293 (2014).

6. Zhao, W. et al. Fullerene-Free Polymer Solar Cells with over $11 \%$ Efficiency and Excellent Thermal Stability. Adv. Mater. 28, 4734-4739 (2016).

7. Li, M. et al. Solution-processed organic tandem solar cells with power conversion efficiencies $>12 \%$. Nat. Photonics 11, 85-90 (2017).

8. Würfel, P. Is an illuminated semiconductor far from thermodynamic equilibrium? Sol. Energy Mater. Sol. Cells 38, 23-28 (1995).

9. Würfel, P. Physics of Solar Cells: From Basic Principles to Advanced Concepts, 2nd Edition. (Wiley$\mathrm{VCH}, 2009)$.

10. Doany, F. E. \& Grischkowsky, D. Measurement of ultrafast hot-carrier relaxation in silicon by thinfilm-enhanced, time-resolved reflectivity. Appl. Phys. Lett. 52, 36-38 (1988).

11. Goldman, J. R. \& Prybyla, J. A. Ultrafast hot-electron dynamics in silicon. Semicond. Sci. Technol. 9, 694 (1994).

12. Bässler, H. Charge Transport in Disordered Organic Photoconductors a Monte Carlo Simulation Study. Phys. Status Solidi B 175, 15-56 (1993).

13. Österbacka, R. et al. Mobility and density relaxation of photogenerated charge carriers in organic materials. Curr. Appl. Phys. 4, 534-538 (2004).

14. Mozer, A. J. et al. Time-dependent mobility and recombination of the photoinduced charge carriers in conjugated polymer/fullerene bulk heterojunction solar cells. Phys. Rev. B 72, 035217 (2005).

15. Devižis, A., Serbenta, A., Meerholz, K., Hertel, D. \& Gulbinas, V. Ultrafast Dynamics of Carrier Mobility in a Conjugated Polymer Probed at Molecular and Microscopic Length Scales. Phys. Rev. Lett. 103, 027404 (2009). 
16. Cabanillas-Gonzalez, J. et al. Photoinduced Transient Stark Spectroscopy in Organic Semiconductors: A Method for Charge Mobility Determination in the Picosecond Regime. Phys. Rev. Lett. 96, 106601 (2006).

17. Ponseca, C. S. et al. Ultrafast Terahertz Photoconductivity of Bulk Heterojunction Materials Reveals High Carrier Mobility up to Nanosecond Time Scale. J. Am. Chem. Soc. 134, 11836-11839 (2012).

18. Devižis, A., Meerholz, K., Hertel, D. \& Gulbinas, V. Hierarchical charge carrier motion in conjugated polymers. Chem. Phys. Lett. 498, 302-306 (2010).

19. Savenije, T. J. et al. Absence of Postnanosecond Charge Carrier Relaxation in Poly(3hexylthiophene)/Fullerene Blends. J. Phys. Chem. Lett. 2, 1368-1371 (2011).

20. Murthy, D. H. K. et al. Origin of Reduced Bimolecular Recombination in Blends of Conjugated Polymers and Fullerenes. Adv. Funct. Mater. 23, 4262-4268 (2013).

21. Mihailetchi, V. D., Koster, L. J. A., Hummelen, J. C. \& Blom, P. W. M. Photocurrent Generation in Polymer-Fullerene Bulk Heterojunctions. Phys. Rev. Lett. 93, 216601 (2004).

22. Vandewal, K. et al. Efficient charge generation by relaxed charge-transfer states at organic interfaces. Nat. Mater. 13, 63-68 (2014).

23. Green, M. A. \& Bremner, S. P. Energy conversion approaches and materials for high-efficiency photovoltaics. Nat. Mater. 16, 23-34 (2017).

24. Yamasaki, S. Optical absorption edge of hydrogenated amorphous silicon studied by photoacoustic spectroscopy. Philos. Mag. Part B 56, 79-97 (1987).

25. Vanecek, M. \& Poruba, A. Fourier-transform photocurrent spectroscopy of microcrystalline silicon for solar cells. Appl. Phys. Lett. 80, 719-721 (2002).

26. Orenstein, J. \& Kastner, M. A. Thermalization and recombination in amorphous semiconductors. Solid State Commun. 40, 85-89 (1981).

27. Simmons, J. G. \& Taylor, G. W. Nonequilibrium Steady-State Statistics and Associated Effects for Insulators and Semiconductors Containing an Arbitrary Distribution of Traps. Phys. Rev. B 4, 502511 (1971).

28. Tiedje, T. Band tail recombination limit to the output voltage of amorphous silicon solar cells. Appl. Phys. Lett. 40, 627-629 (1982).

29. Blakesley, J. C. \& Neher, D. Relationship between energetic disorder and open-circuit voltage in bulk heterojunction organic solar cells. Phys. Rev. B 84, 075210 (2011).

30. Osterloh, F. E. Maximum Theoretical Efficiency Limit of Photovoltaic Devices: Effect of Band Structure on Excited State Entropy. J. Phys. Chem. Lett. 5, 3354-3359 (2014).

31. Vandewal, K., Tvingstedt, K., Gadisa, A., Inganäs, O. \& Manca, J. V. On the origin of the opencircuit voltage of polymer-fullerene solar cells. Nat. Mater. 8, 904-909 (2009). 
32. Vandewal, K., Tvingstedt, K., Gadisa, A., Inganäs, O. \& Manca, J. V. Relating the open-circuit voltage to interface molecular properties of donor:acceptor bulk heterojunction solar cells. Phys. Rev. B 81, 125204 (2010).

33. Yao, J. et al. Quantifying Losses in Open-Circuit Voltage in Solution-Processable Solar Cells. Phys. Rev. Appl. 4, 014020 (2015).

34. Tang, C. W. Two-layer organic photovoltaic cell. Appl. Phys. Lett. 48, 183-185 (1986).

35. Mikhnenko, O. V., Blom, P. W. M. \& Nguyen, T.-Q. Exciton diffusion in organic semiconductors. Energy Environ. Sci. 8, 1867-1888 (2015).

36. Yu, G., Gao, J., Hummelen, J. C., Wudl, F. \& Heeger, A. J. Polymer Photovoltaic Cells: Enhanced Efficiencies via a Network of Internal Donor-Acceptor Heterojunctions. Science 270, 1789-1791 (1995).

37. Halls, J. J. M. et al. Efficient photodiodes from interpenetrating polymer networks. Nature 376, 498-500 (1995).

38. Sariciftci, N. S., Smilowitz, L., Heeger, A. J. \& Wudl, F. Photoinduced Electron Transfer from a Conducting Polymer to Buckminsterfullerene. Science 258, 1474-1476 (1992).

39. Gélinas, S. et al. Ultrafast Long-Range Charge Separation in Organic Semiconductor Photovoltaic Diodes. Science 343, 512-516 (2014).

40. Vandewal, K. et al. Quantification of Quantum Efficiency and Energy Losses in Low Bandgap Polymer:Fullerene Solar Cells with High Open-Circuit Voltage. Adv. Funct. Mater. 22, 3480-3490 (2012).

41. Liu, J. et al. Fast charge separation in a non-fullerene organic solar cell with a small driving force. Nat. Energy 1, 16089 (2016).

42. Tang, Z. et al. Semi-Transparent Tandem Organic Solar Cells with $90 \%$ Internal Quantum Efficiency. Adv. Energy Mater. 2, 1467-1476 (2012).

43. Park, S. H. et al. Bulk heterojunction solar cells with internal quantum efficiency approaching 100\%. Nat. Photonics 3, 297-302 (2009).

44. Burke, T. M. \& McGehee, M. D. How High Local Charge Carrier Mobility and an Energy Cascade in a Three-Phase Bulk Heterojunction Enable $>90 \%$ Quantum Efficiency. Adv. Mater. 26, 1923-1928 (2014).

45. Tvingstedt, K. et al. Electroluminescence from Charge Transfer States in Polymer Solar Cells. J. Am. Chem. Soc. 131, 11819-11824 (2009).

46. Tvingstedt, K., Vandewal, K., Zhang, F. \& Inganäs, O. On the Dissociation Efficiency of Charge Transfer Excitons and Frenkel Excitons in Organic Solar Cells: A Luminescence Quenching Study. J. Phys. Chem. C 114, 21824-21832 (2010).

47. Vandewal, K., Tvingstedt, K. \& Inganäs, O. Polarization anisotropy of charge transfer absorption and emission of aligned polymer:fullerene blend films. Phys. Rev. B 86, 035212 (2012). 
48. Vandewal, K. et al. Increased Open-Circuit Voltage of Organic Solar Cells by Reduced DonorAcceptor Interface Area. Adv. Mater. 26, 3839-3843 (2014).

49. Scher, H. \& Montroll, E. W. Anomalous transit-time dispersion in amorphous solids. Phys. Rev. $B$ 12, 2455-2477 (1975).

50. Tiedje, T. \& Rose, A. A physical interpretation of dispersive transport in disordered semiconductors. Solid State Commun. 37, 49-52 (1981).

51. Devižis, A., Hertel, D., Meerholz, K., Gulbinas, V. \& Moser, J.-E. Time-independent, high electron mobility in thin PC61BM films: Relevance to organic photovoltaics. Org. Electron. 15, 3729-3734 (2014).

52. Ponseca, C. S. et al. Electron and Hole Contributions to the Terahertz Photoconductivity of a Conjugated Polymer:Fullerene Blend Identified. J. Phys. Chem. Lett. 3, 2442-2446 (2012).

53. Sher, M.-J. et al. Time- and Temperature-Independent Local Carrier Mobility and Effects of Regioregularity in Polymer-Fullerene Organic Semiconductors. Adv. Electron. Mater. (2016). doi:10.1002/aelm.201500351

54. Pranculis, V. et al. Charge Carrier Generation and Transport in Different Stoichiometry APFO3:PC61BM Solar Cells. J. Am. Chem. Soc. 136, 11331-11338 (2014).

55. Melianas, A. et al. Dispersion-Dominated Photocurrent in Polymer:Fullerene Solar Cells. Adv. Funct. Mater. 24, 4507-4514 (2014).

56. Melianas, A. et al. Photogenerated Carrier Mobility Significantly Exceeds Injected Carrier Mobility in Organic Solar Cells. Adv. Energy Mater. (2017). doi:10.1002/aenm.201602143

57. Melianas, A. et al. Photo-generated carriers lose energy during extraction from polymerfullerene solar cells. Nat. Commun. 6, 8778 (2015).

58. Baranovskii, S. D. Theoretical description of charge transport in disordered organic semiconductors. Phys. Status Solidi B 251, 487-525 (2014).

59. Tanase, C., Meijer, E. J., Blom, P. W. M. \& de Leeuw, D. M. Unification of the Hole Transport in Polymeric Field-Effect Transistors and Light-Emitting Diodes. Phys. Rev. Lett. 91, 216601 (2003).

60. Coehoorn, R., Pasveer, W. F., Bobbert, P. A. \& Michels, M. A. J. Charge-carrier concentration dependence of the hopping mobility in organic materials with Gaussian disorder. Phys. Rev. B 72, 155206 (2005).

61. Pasveer, W. F. et al. Unified Description of Charge-Carrier Mobilities in Disordered Semiconducting Polymers. Phys. Rev. Lett. 94, 206601 (2005).

62. Craciun, N. I., Wildeman, J. \& Blom, P. W. M. Universal Arrhenius Temperature Activated Charge Transport in Diodes from Disordered Organic Semiconductors. Phys. Rev. Lett. 100, 056601 (2008).

63. Anderson, P. W. Absence of Diffusion in Certain Random Lattices. Phys. Rev. 109, 1492-1505 (1958). 
64. Miller, A. \& Abrahams, E. Impurity Conduction at Low Concentrations. Phys. Rev. 120, 745-755 (1960).

65. Gill, W. D. Drift mobilities in amorphous charge-transfer complexes of trinitrofluorenone and poly-n-vinylcarbazole. J. Appl. Phys. 43, 5033-5040 (1972).

66. van Eersel, H., Janssen, R. A. J. \& Kemerink, M. Mechanism for Efficient Photoinduced Charge Separation at Disordered Organic Heterointerfaces. Adv. Funct. Mater. 22, 2700-2708 (2012).

67. Howard, I. A., Etzold, F., Laquai, F. \& Kemerink, M. Nonequilibrium Charge Dynamics in Organic Solar Cells. Adv. Energy Mater. 4, 1301743 (2014).

68. Wang, E. et al. Conformational Disorder Enhances Solubility and Photovoltaic Performance of a Thiophene-Quinoxaline Copolymer. Adv. Energy Mater. 3, 806-814 (2013).

69. Abramavicius, V. et al. Role of coherence and delocalization in photo-induced electron transfer at organic interfaces. Sci. Rep. 6, 32914 (2016).

70. Noriega, R., Salleo, A. \& Spakowitz, A. J. Chain conformations dictate multiscale charge transport phenomena in disordered semiconducting polymers. Proc. Natl. Acad. Sci. 110, 16315-16320 (2013).

71. Němec, H. et al. Ultrafast conductivity in a low-band-gap polyphenylene and fullerene blend studied by terahertz spectroscopy. Phys. Rev. B 79, 245326 (2009).

72. Lane, P. A., Cunningham, P. D., Melinger, J. S., Esenturk, O. \& Heilweil, E. J. Hot photocarrier dynamics in organic solar cells. Nat. Commun. 6, 7558 (2015).

73. Vithanage, D. A. et al. Visualizing charge separation in bulk heterojunction organic solar cells. Nat. Commun. 4, 2334 (2013).

74. Devizis, A., Meerholz, K., Hertel, D. \& Gulbinas, V. Ultrafast charge carrier mobility dynamics in poly(spirobifluorene-co-benzothiadiazole): Influence of temperature on initial transport. Phys. Rev. B 82, 155204 (2010).

75. Tang, Z. et al. Interlayer for Modified Cathode in Highly Efficient Inverted ITO-Free Organic Solar Cells. Adv. Mater. 24, 554-558 (2012).

76. Christ, N., Kettlitz, S. W., Züfle, S., Valouch, S. \& Lemmer, U. Nanosecond response of organic solar cells and photodiodes: Role of trap states. Phys. Rev. B 83, 195211 (2011).

77. Kettlitz, S. W. et al. Eliminating RC-Effects in Transient Photocurrent Measurements on Organic Photodiodes. IEEE Photonics Technol. Lett. 25, 682-685 (2013).

78. Gallaher, J. K. et al. Spectroscopically tracking charge separation in polymer:fullerene blends with a three-phase morphology. Energy Environ. Sci. 8, 2713-2724 (2015).

79. Barker, A. J., Chen, K. \& Hodgkiss, J. M. Distance Distributions of Photogenerated Charge Pairs in Organic Photovoltaic Cells. J. Am. Chem. Soc. 136, 12018-12026 (2014).

80. Kumar, A. et al. Interface limited charge extraction and recombination in organic photovoltaics. Energy Environ. Sci. 7, 2227-2231 (2014). 
81. Andersson, L. M. et al. Unified Study of Recombination in Polymer:Fullerene Solar Cells Using Transient Absorption and Charge-Extraction Measurements. J. Phys. Chem. Lett. 4, 2069-2072 (2013).

82. Beiley, Z. M. et al. Morphology-Dependent Trap Formation in High Performance Polymer Bulk Heterojunction Solar Cells. Adv. Energy Mater. 1, 954-962 (2011).

83. Němec, H., Kužel, P. \& Sundström, V. Charge transport in nanostructured materials for solar energy conversion studied by time-resolved terahertz spectroscopy. J. Photochem. Photobiol. Chem. 215, 123-139 (2010).

84. Glover, R. E. \& Tinkham, M. Conductivity of Superconducting Films for Photon Energies between 0.3 and $40 \mathrm{kT}$. Phys. Rev. 108, 243-256 (1957).

85. Wang, C., Duong, D. T., Vandewal, K., Rivnay, J. \& Salleo, A. Optical measurement of doping efficiency in poly(3-hexylthiophene) solutions and thin films. Phys. Rev. B 91, 085205 (2015).

86. Pasveer, W. F., Bobbert, P. A. \& Michels, M. A. J. Universality of AC conductivity: Random siteenergy model with Fermi statistics. Phys. Rev. B 74, 165209 (2006).

87. Vukmirović, N., Ponseca, C. S., Němec, H., Yartsev, A. \& Sundström, V. Insights into the Charge Carrier Terahertz Mobility in Polyfluorenes from Large-Scale Atomistic Simulations and TimeResolved Terahertz Spectroscopy. J. Phys. Chem. C 116, 19665-19672 (2012).

88. Hilt, O. \& Siebbeles, L. D. A. Time and frequency dependent charge carrier mobility on onedimensional chains with energetic disorder for polaron and Miller-Abrahams type hopping. Chem. Phys. 229, 257-263 (1998).

89. Savenije, T. J., Ferguson, A. J., Kopidakis, N. \& Rumbles, G. Revealing the Dynamics of Charge Carriers in Polymer:Fullerene Blends Using Photoinduced Time-Resolved Microwave Conductivity. J. Phys. Chem. C 117, 24085-24103 (2013).

90. Juška, G. et al. Charge transport in $\pi$-conjugated polymers from extraction current transients. Phys. Rev. B 62, R16235-R16238 (2000).

91. Baumann, A., Lorrmann, J., Rauh, D., Deibel, C. \& Dyakonov, V. A New Approach for Probing the Mobility and Lifetime of Photogenerated Charge Carriers in Organic Solar Cells Under Real Operating Conditions. Adv. Mater. 24, 4381-4386 (2012).

92. Mott, N. F. \& Gurney, R. W. Electronic Processes in lonic Crystals. (Oxford University Press, 1940).

93. Lampert, M. A. Simplified Theory of Space-Charge-Limited Currents in an Insulator with Traps. Phys. Rev. 103, 1648-1656 (1956).

94. Murgatroyd, P. N. Theory of space-charge-limited current enhanced by Frenkel effect. J. Phys. Appl. Phys. 3, 151 (1970).

95. Gao, F. et al. The Effect of Processing Additives on Energetic Disorder in Highly Efficient Organic Photovoltaics: A Case Study on PBDTTT-C-T:PC71BM. Adv. Mater. 27, 3868-3873 (2015).

96. Mauer, R., Kastler, M. \& Laquai, F. The Impact of Polymer Regioregularity on Charge Transport and Efficiency of P3HT:PCBM Photovoltaic Devices. Adv. Funct. Mater. 20, 2085-2092 (2010). 
97. Mukherjee, S., Jiao, X. \& Ade, H. Charge Creation and Recombination in Multi-Length Scale Polymer:Fullerene BHJ Solar Cell Morphologies. Adv. Energy Mater. (2016).

doi:10.1002/aenm.201600699

98. Mukherjee, S., Proctor, C. M., Bazan, G. C., Nguyen, T.-Q. \& Ade, H. Significance of Average Domain Purity and Mixed Domains on the Photovoltaic Performance of High-Efficiency SolutionProcessed Small-Molecule BHJ Solar Cells. Adv. Energy Mater. (2015). doi:10.1002/aenm.201500877

99. Collins, B. A. et al. Absolute Measurement of Domain Composition and Nanoscale Size Distribution Explains Performance in PTB7:PC71BM Solar Cells. Adv. Energy Mater. 3, 65-74 (2013).

100. Rivnay, J., Mannsfeld, S. C. B., Miller, C. E., Salleo, A. \& Toney, M. F. Quantitative Determination of Organic Semiconductor Microstructure from the Molecular to Device Scale. Chem. Rev. 112, 5488-5519 (2012).

101. Proctor, C. M. et al. Understanding Charge Transport in Molecular Blend Films in Terms of Structural Order and Connectivity of Conductive Pathways. Adv. Energy Mater. (2016). doi:10.1002/aenm.201502285

102. Foster, S. et al. Electron Collection as a Limit to Polymer:PCBM Solar Cell Efficiency: Effect of Blend Microstructure on Carrier Mobility and Device Performance in PTB7:PCBM. Adv. Energy Mater. (2014). doi:10.1002/aenm.201400311

103. Guo, X. et al. Influence of D/A Ratio on Photovoltaic Performance of a Highly Efficient Polymer Solar Cell System. Adv. Mater. 24, 6536-6541 (2012).

104. Frankevich, E., Maruyama, Y. \& Ogata, H. Mobility of charge carriers in vapor-phase grown C60 single crystal. Chem. Phys. Lett. 214, 39-44 (1993).

105. Sakai, J., Taima, T. \& Saito, K. Efficient oligothiophene:fullerene bulk heterojunction organic photovoltaic cells. Org. Electron. 9, 582-590 (2008).

106. Fowler, R. H. \& Nordheim, L. Electron Emission in Intense Electric Fields. Proc. R. Soc. Lond. Math. Phys. Eng. Sci. 119, 173-181 (1928).

107. Tuladhar, S. M. et al. Ambipolar Charge Transport in Films of Methanofullerene and Poly(phenylenevinylene)/Methanofullerene Blends. Adv. Funct. Mater. 15, 1171-1182 (2005).

108. Gadisa, A. et al. Bipolar Charge Transport in Fullerene Molecules in a Bilayer and Blend of Polyfluorene Copolymer and Fullerene. Adv. Mater. 22, 1008-1011 (2010).

109. Jamieson, F. C. et al. Fullerene crystallisation as a key driver of charge separation in polymer/fullerene bulk heterojunction solar cells. Chem. Sci. 3, 485-492 (2012).

110. Sweetnam, S. et al. Characterization of the Polymer Energy Landscape in Polymer:Fullerene Bulk Heterojunctions with Pure and Mixed Phases. J. Am. Chem. Soc. 136, 14078-14088 (2014).

111. Groves, C. Developing understanding of organic photovoltaic devices: kinetic Monte Carlo models of geminate and non-geminate recombination, charge transport and charge extraction. Energy Environ. Sci. 6, 3202-3217 (2013). 
112. Marcus, R. A. On the Theory of Oxidation-Reduction Reactions Involving Electron Transfer. J. Phys. Chem. 67, 853-857 (1963).

113. Venkateshvaran, D. et al. Approaching disorder-free transport in high-mobility conjugated polymers. Nature 515, 384-388 (2014).

114. Himmelberger, S. \& Salleo, A. Engineering semiconducting polymers for efficient charge transport. MRS Commun. 5, 383-395 (2015).

115. Würfel, P. Solar energy conversion with hot electrons from impact ionisation. Sol. Energy Mater. Sol. Cells 46, 43-52 (1997). 


\section{Appendix}

\section{Investigated materials}

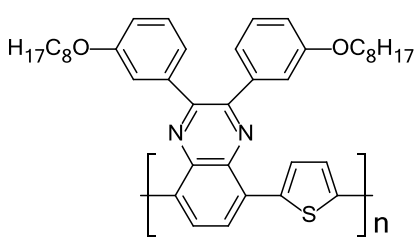

TQ1

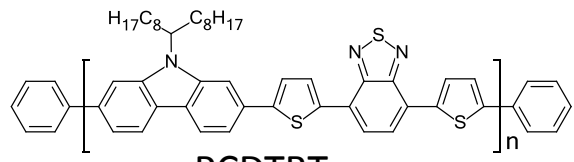

PCDTBT

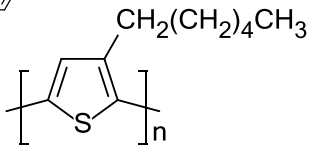

rrP3HT

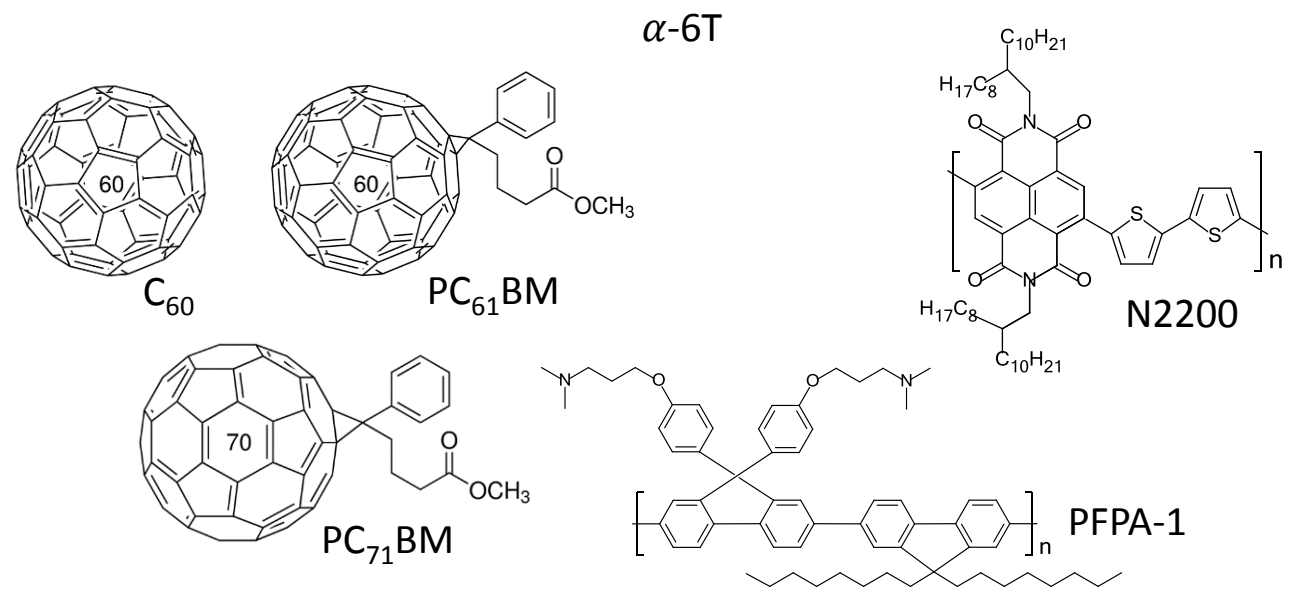

Figure A1. Chemical structures of the donor and acceptor materials used in this thesis. The interface material PFPA-1 is also shown.

Table A1. Full material names.

\begin{tabular}{|c|c|}
\hline TQ1 & Poly[2,3-bis-(3-octyloxyphenyl)quinoxaline-5,8-diyl-alt-thiophene-2,5-diyl] \\
\hline PCDTBT & $\begin{array}{l}\text { Poly[N-11'”-henicosanyl-2,7-carbazole-alt-5,5-(4',7'-di-2-thienyl-2',1',3'- } \\
\text { benzothiadiazole) }]\end{array}$ \\
\hline $\mathrm{rrP3HT}$ & Regioregular Poly(3-hexylthiophene-2,5-diyl) \\
\hline$\alpha-6 \mathrm{~T}$ & Alpha-sexithiophene \\
\hline $\mathrm{C}_{60}$ & Buckminsterfullerene \\
\hline $\mathrm{PC}_{61} \mathrm{BM}$ & {$[6,6]$-phenyl- $\mathrm{C}_{61}$-butyric acid methyl ester } \\
\hline $\mathrm{PC}_{71} \mathrm{BM}$ & {$[6,6]$-phenyl-C 71 -butyric acid methyl ester } \\
\hline N2200 & $\begin{array}{l}\operatorname{Poly}\left(N, N^{\prime} \text {-bis(2-octyldodecyl)naphthalene-1,4,5,8-bis(dicarboximide)-2,6-diyl-alt- }\right. \\
\text { 2,2'-bithiophene-5,5'-diyl) }\end{array}$ \\
\hline PFPA-1 & $\begin{array}{l}\text { Poly(3,3'-([(9',9'-dioctyl-9H,9'H-[2,2'-bifluorene]-9,9-diyl)bis(4,1- } \\
\text { phenylene)]bis(oxy))bis(N,N-dimethylpropan-1-amine)) }\end{array}$ \\
\hline
\end{tabular}





\section{Papers}

The articles associated with this thesis have been removed for copyright reasons. For more details about these see:

http://urn.kb.se/resolve?urn=urn:nbn:se:liu:diva-136479 